\title{
Therapeutic approaches for the protection of neurological synapses in autoimmunity
}

Citation for published version (APA):

Losen, M. R. (2006). Therapeutic approaches for the protection of neurological synapses in autoimmunity. [Doctoral Thesis, Maastricht University]. Universiteit Maastricht. https://doi.org/10.26481/dis.20060601ml

Document status and date:

Published: 01/01/2006

DOI:

10.26481/dis.20060601ml

Document Version:

Publisher's PDF, also known as Version of record

\section{Please check the document version of this publication:}

- A submitted manuscript is the version of the article upon submission and before peer-review. There can be important differences between the submitted version and the official published version of record.

People interested in the research are advised to contact the author for the final version of the publication, or visit the DOI to the publisher's website.

- The final author version and the galley proof are versions of the publication after peer review.

- The final published version features the final layout of the paper including the volume, issue and page numbers.

Link to publication

\footnotetext{
General rights rights.

- You may freely distribute the URL identifying the publication in the public portal. please follow below link for the End User Agreement:

www.umlib.nl/taverne-license

Take down policy

If you believe that this document breaches copyright please contact us at:

repository@maastrichtuniversity.nl

providing details and we will investigate your claim.
}

Copyright and moral rights for the publications made accessible in the public portal are retained by the authors and/or other copyright owners and it is a condition of accessing publications that users recognise and abide by the legal requirements associated with these

- Users may download and print one copy of any publication from the public portal for the purpose of private study or research.

- You may not further distribute the material or use it for any profit-making activity or commercial gain

If the publication is distributed under the terms of Article $25 \mathrm{fa}$ of the Dutch Copyright Act, indicated by the "Taverne" license above, 


\section{Therapeutic Approaches for the Protection of Neurological Synapses in Autoimmunity}

Mario Losen 
(c) Mario Losen, Maastricht 2006

Typesetting and coverdesign by Michał Stawiríski

Printed in Poland

ISBN-10: 90-9020729-5

ISBN-13: 978-90-9020729-2 


\title{
Therapeutic Approaches for the Protection of Neurological Synapses in Autoimmunity
}

\author{
Proefschrift
}

ter verkrijging van de graad van doctor aan de Universiteit Maastricht op gezag van de Rector Magnificus, Prof. Mr. G.P.M.F. Mols

volgens het besluit van het College van Decanen

in het openbaar te verdedigen

op donderdag 1 juni 2006 om 14.00 uur

door

Mario René Losen

geboren op 21 augustus 1974 te Haselünne 


\section{Promotor:}

Prof. Dr. H.W.M. Steinbusch

\section{Co-promotor:}

Dr. M.H. De Baets

\section{Beoordelingscommissie:}

Prof. Dr. W.A. Buurman (voorzitter)

Prof. Dr. M.J.A.P. Daemen

Prof. Dr. F.C.S. Ramaekers

Prof. Dr. P. Stinissen (University of Hasselt-Diepenbeek)

Prof. Dr. J.H. Wokke (University of Utrecht)

The studies described in this thesis were supported by grants from the Prinses Beatrix Fonds, LAssociation Française contre les Myopathies and the project grant "QLG3-CT-2001-00225" of the European Community program "quality of life and management of living resources". 
Es ist nicht genug, zu wissen, man muß auch anwenden; es ist nicht genug, zu wollen, man muß auch tun.

Johann Wolfgang von Goethe 



\section{Contents}

Introduction

Chapter 1

The effect of anti-acetylcholine receptor antibodies in experimental autoimmune myasthenia gravis

Increased expression of rapsyn in muscles prevents

Chapter 2 acetylcholine receptor loss in experimental

autoimmune myasthenia gravis

Rapsyn gene therapy of muscles increases

Chapter 3 acetyicholine receptor levels in chronic experimental autoimmune myasthenia gravis

Chapter 4 Experimental autoimmune myasthenia gravis in rhesus monkeys

Passive transfer of a human IgG4 anti-AChR

Chapter 5 antibody prevents experimental autoimmune

myasthenia gravis in rhesus monkeys

Chapter 6 The features of myasthenia gravis

Summary and general discussion

Samenvatting en algemene discussie

Publications

Curriculum Vitae 


\section{Abbreviations}

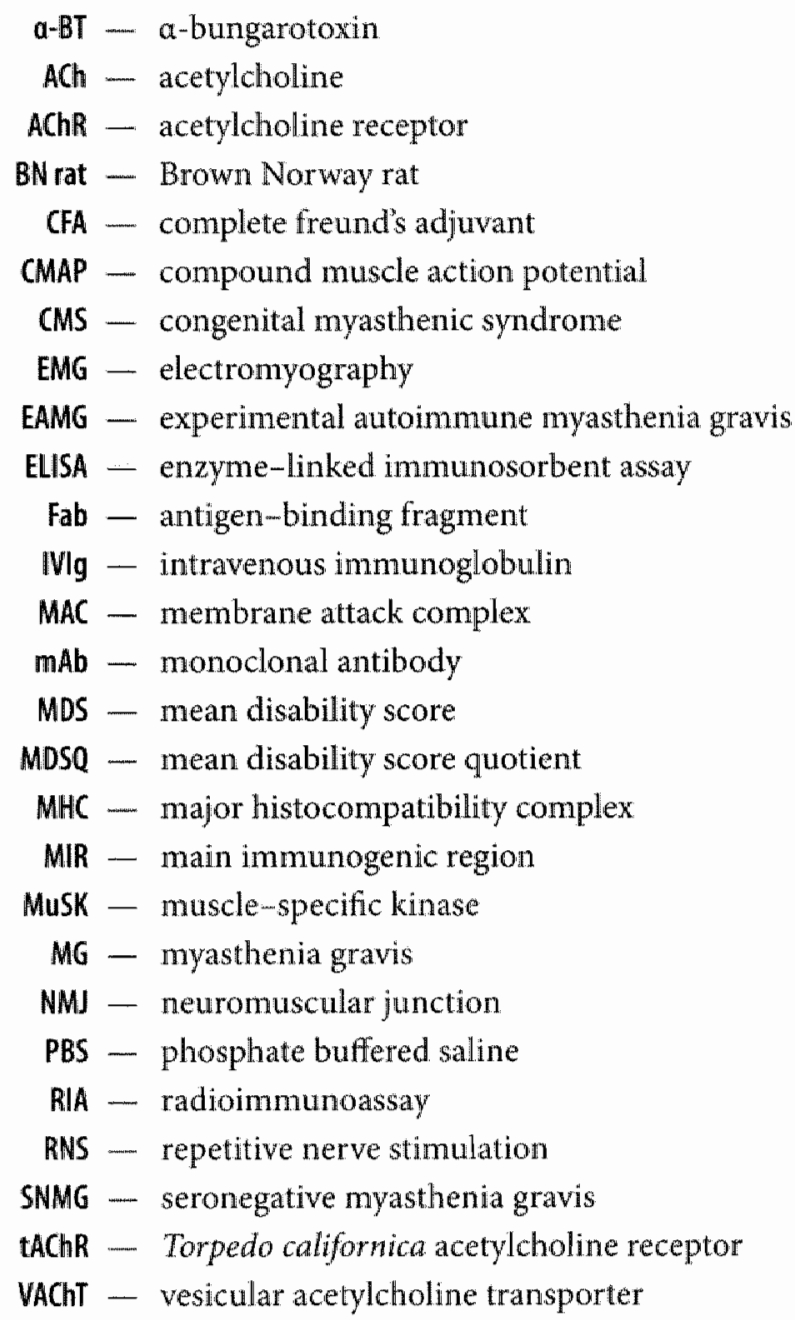




\section{Introduction}

The immune system protects the body against pathogens by eliminating non-self antigens. Immune responses are highly regulated to avoid hypersensitivity and wasted resources when the pathogens are eliminated. In spite of this regulation and mechanisms for clonal deletion of many self-reactive lymphocytes, the immune system occasionally attacks self-tissues and produces autoimmunity. Currently, autoimmune diseases affect $5-7 \%$ of the population. Many autoimmune diseases are caused by antibodies to cells surface receptors (type II hypersensitivity reactions). When antibodies are directed against nerve and synapse proteins, neurological disorders occur. The first neurological disease recognized to have an autoimmune mechanism was myasthenia gravis (MG). Similar mechanisms have been identified subsequently in other diseases of the peripheral nervous system such as the Lambert-Eaton myasthenic syndrome and the Guillain-Barre syndrome. Moreover, there is accumulating evidence that autoantibodies can also cause or aggravate disorders of the central nervous system, including a number of paraneoplastic syndromes and multiple sclerosis.

The characteristic chronic muscle weakness of $85 \% \mathrm{MG}$ patients is caused by antibodies against the nicotinic muscle acetylcholine receptor (AChR) resulting in the destruction of the postsynaptic membrane of the neuromuscular junction (NMJ) and functional denervation. Because of the early discovery of this autoantigen, the effector mechanisms of anti-AChR antibodies are now well known. The animal model of $M G$, termed experimental autoimmune myasthenia gravis (EAMG) has been very valuable for our understanding of the disease process because of its reproducibility and similarity to the human disease (Chapters 1 and 4). Additionally, in approximately one third of MG patients without anti $\sim A C h R$ antibodies (seronegative patients), antibodies against the muscle specific kinase (MUSK) have been found. This group of patients was previously diagnosed only on the basis of clinical symptoms and the effector mechanism of anti-MuSK antibodies is now under investigation. For this purpose, it is important to characterize the specific disease symptoms of this subgroup of $M G$ patients (Chapter 6).

The neuromuscular junction responds to the autoantibody attack by changing the expression of a number of proteins. The AChR and complement regulatory proteins are upregulated to compensate for receptor loss and limit further damage to the NMJ. Many changes, however, are a consequence of functional denervation of the muscle with concomitant loss of postsynaptic specialization. Thus, the study of the underlying mechanisms of maintenance of postsynaptic organization is important for understanding the susceptibility to the autoantibody attack and the recovery of the damaged postsynaptic membrane.

Current therapies for autoimmune diseases aim at suppressing the immune system by removal of antibodies using plasmapheresis, or by pharmacological inhibition of lymphocyte 
proliferation. However these therapies are not specific for the autoimmune response, and have to be carefilly balanced to prevent an unacceptable increase of susceptibility to infections and cancer. For this reason complete remission of autoimmune diseases is difficult to achieve by general immunosuppression. To avoid this intrinsic dilemma, antigen specific therapies are required. These can target the $T-$ and $B$-cell clones that mount the immune response, the autoantigen-specific antibodies or the target tissue. Gene therapy of the target tissue is a promising therapeutic approach for protecting the autoantigen. Possible protective molecules include anti-inflammatory cytokines and complement regulatory proteins. "Regenerative gene therapy" aims at supplying critical proteins required for the protection and recovery of the target tissue (chapters 2 and 3). A critical limitation of gene therapy is currently the lack of safe and efficient vectors. Conversely, immunotherapy with monoclonal human antibodies has been proven to be efficacious and side effects are usually mild. Current treatments using monoclonal antibodies in autoimmunity are not antigen specific, but have a general immunosuppressive effect. Autoantigen-specific immunoglobulins without pathogenic properties could specifically block important epitopes on the target tissue and thereby prevent auto-antibody binding (Chaper 5).

Chapter $\mathbb{1}$ is an introduction to the animal models of myasthenia gravis and the corresponding disease mechanisms.

Chapter 2 describes a therapeutic approach to prevent passive transfer autoimmune myasthenia gravis using rapsyn DNA electroporation of the target organ.

Chapter 3 analyses the effect of rapsyn gene therapy in ongoing chronic experimental autoimmune myasthenia gravis.

Chapter 4 reviews the use of non-human primates for the research of myasthenia gravis.

Chapter 5 describes an immunotherapy in thesus monkeys using AChR specific monoclonal burman $\lg G 4$.

Chapter 6 analyses the pathogenic effects of anti MuSK antibodies in MG patients. 


\section{Chapter 1}

The Effect of Anti-Acetylcholine Receptor Antibodies

in Experimental Autoimmune

Myasthenia Gravis

Mario Losen, Pilar Martínez-Martínez, Marc H. De Baets 


\section{Introduction}

Experimental autoimmune myasthenia gravis (EAMG) can be induced in a large number of animal species by active immunisation (AI) with $\mathrm{AChR}$, by passive transfer (PT) of anti-AChR antibodies, by autologous bone marrow transplantation and cyclosporin (BMT-Cy), or occur spontaneously. Depending on the model used, different immunological mechanisms are operational. In the $\mathrm{AI}$ model, the $\mathrm{T}$ cell is pivotal in directing the anti-AChR antibody production towards pathogenic, that is, cross-linking and complement-fixing antibodies. Injection of anti-AChR antibodies alone suffices to induce EAMG, excluding the role of specific cell-mediated immune responses in the effector phase of the disease. Aged animals are resistant to the induction of AI and PT EAMG. This resistance is localised at the postsynaptic membrane containing more AChR-anchoring proteins, including s-laminin (laminin $a 1 \beta 2 \gamma 1$ ) and rapsyn in aged animals. In BMT-CyA EAMG, a dysregulation of the immune system in the absence of immunisation is capable of inducing myasthenia. The role of these animal models in relation to pathogenesis and immunotherapy is discussed (Table 1 ).

Table 1. EAMG models

EAMG after active immunisation
Passive transfer EAMG
EAMG after bone inarrow transpliantation
EAMG after thymus grafting
Spontaneous myasthenia
EAMG inn transgenic animais

\section{EAMG after active immunisation}

Patrick and Lindstrom immunised rabbits with purified AChR from the electric organ of electric eels to obtain antibodies for their biochemical analysis of AChR. All animals became paralysed and eventually died (Patrick and Lindstrom, 1973). Active immunisation of experimental animals (including rabbits, rats, mice, guinea pigs, monkeys, and goats) with AChR with or without adjuvant induced chronic EAMG within 30 days after immunisation. The animals mount an active immune response against injected $A C h R$. The disease is induced by antibodies cross-reacting with the animals' own AChR. In this model, many immunological aspects of MG can be studied, such as AChR processing, APC presentation, the role of $\mathrm{MHC}_{3}$, T cell subsets, and cytokines (Christadoss et al., 2000), as well as many nonimmune-mediated applications that are relevant to human $M G$. 


\section{Passive transfer EAMG}

Injection of EAMG or MG serum, or monodonal antibodies against the main inmunogenic region (MIR) or against the ACh binding sites, in experimental animals induces MG within hours or days (Hoedemaekers et al., 1997c). Antibodies against the ACh binding sites induce a curare-like effect within 15-30 min (Balass et al., 1993). Anti-AChR binding site antibodies block the ligand-induced opening of the ion channel. Antibodies against the MIR induce myasthenia within $8-48$ h depending on the dose and affinity of the antibody for AChR. The immunopathological mechanisms are antigenic modulation and complement-mediated focal lysis of the postsynaptic membrane for anti-MIR antibodies (Lindstrom, 2000). This pas sive transfer model is relevant for $M G$ in order to study the effector phase of the disease.

\section{EAMG after bone marrow transplantation}

After bone marrow transplantation (BMT), humans and experimental animals developed a graft versus host disease and occasionally MG. In a rat model originally developed by Hess and colleagues (reviewed by Damoiseaux, 2002), Lewis rats are lethally irradiated and rescued by a syngeneic BMT. The animals are injected with a low dose of cyclosporin A for 4 weeks. Two weeks after stopping cyclosporin A, the animals develop a scleroderma-like skin disease. In the course of these studies, two animals out of several hundred were observed that spontaneously developed MG without injection of any antigen or adjuvant. Both rats had circulating anti-rat AChR antibodies, increased jitter and single-fiber EMG, and AChR loss (G. Bos et al., unpublished observations). The mechanism of this autoimmune disease is the result of defective $\mathrm{T}$ cell development, including aberrant selection in the thymus and disturbed $T$ cell balances in the periphery. Cyclosporin $A$ is required for the intrathymic generation of autoreactive cells. Fewer cortical thymocytes are surviving positive selection and fewer medullary thymocytes are eliminated during negative selection-induced maturation arrest of the CD4t and CD8+ double-positive thymocytes (Damoiseaux, 2002). This model is probably relevant for $M G$ after BMT and for the study of the role of the thymus in $M G$. However, the incidence of EAMG in the BMT model is too low for a more systematic study.

\section{EAMG after thymus grafting}

Thymus explants from MG patients with follicular hyperplasia, but not thymoma, induce MG when transplanted under the kidney capsule in SCID mice. Anti-human AChR antibodies produced by the thymic transplant cross-react with mouse $A C h R$, which causes AChR loss and muscle weakness. The thymus contains all components to effectuate myasthenia, including AChR-expressing myoid cells, antigen-presenting cells, and $\mathrm{B}$ and $\mathrm{T}$ cells (Monfardini et al., 2002; Schonbeck et al., 1992). This MG model is relevant for the study of the role of the thymus and its cellular components. 


\section{Spontaneous myasthenia}

MG spontaneously occurs in cats, dogs, and horses. Similar to the human disease, thymic abnormalities are seen and circulating anti-AChR antibodies can be detected by radioimmunoassay (Shelton, 2002). The immunological mechanisms operational in these models are probably identical to human MG. The study of spontaneous $M G$ in animals is very relevant for human MG. Unfortunately, few animals are available for experimental studies, mostly because the owners do not like to donate their pets for scientific studies.

\section{EAMG in transgenic animals}

Introducing or silencing immunological relevant genes in mice prior to immunisation with AChR can pinpoint the role of these genes in EAMG and MG. Elimination of the genes that are relevant for $\mathrm{APC} / \mathrm{T}$ cell interaction, including $\mathrm{MHC} I \mathbb{I}$ and $\mathrm{CD} 4$, results in a loss of susceptibility to disease. In the absence of the CD8 molecule, mice dewelop EAMG, which indicates that cytotoxic $T$ cells are probably not involved in the pathogenesis of the disease. NKI knockout mice fail to develop EAMG (Christadoss et al., 2000). Mice transgenic for the human MHC molecule (Yang et al.,2002) are relevant for the study of the specificity of the $T$ cell response and the susceptibility to the disease. Unfortunately, these animals do not express human $\mathrm{T}$ cell receptor molecules. Mice transgenic for the human IgG gene allow us to study the role of human immunoglobulins in EAMG (Stassen et al., 2003). In another MG model, no immunisation is required. Targeting the interferon $\gamma$ gene to the postsynaptic membrane using the promoter of the $\gamma$ subunit of AChR results in a local inflammation of the endplate and muscular weakness ( $\mathrm{Gu}$ et al, 1995; Weigle, 1980). The animals in this model develop serum antibodies against an $87-\mathrm{kD}$ a protein at the endplates, but not against AChR. Therefore, this model is not suitable for the study of MG.

\section{Immunoregulation at the T cell level}

MG and EAMG are typical antibody-mediated diseases, but the production of the pathogenic antibodies by $\mathrm{B}$ cells depends on $\mathrm{T}$ helper cells. The degree of tolerance in both $\mathrm{T}$ and $B$ cell compartments is dependent upon the concentration of the self-antigen in the microenvironment. If the concentration of the self-antigen, for example albumin, is high, both $\mathrm{T}$ and $\mathrm{B}$ cells are tolerant. If the concentration is low, as for thyroglobulin, only $\mathrm{T}$ cells are tolerant (Weigle, 1980). Immunisation of animals with syngeneic thyroglobulin, which is normally circulating in nanogram amounts (De Baets et al., 1983), does not result in a $\mathrm{T}$ cell proliferative response (Table 2). There is no tolerance if the self-antigen is not circulating in the microemvironment as is the case for AChR or myelin basic protein (MBP). Indeed, injection of nanogram amounts of syngeneic rat $A C h R$ in Lewis rats induces a vigorous immune response as judged by a $\mathrm{T}$ cell proliferation assay (Table 3 ). 
Table 2. Thyroglobulin-specific proliferation of immune lymph node cells

\begin{tabular}{cccc}
\hline \multirow{2}{*}{ Animal } & & \multicolumn{2}{c}{ Simulation index } \\
\cline { 2 - 4 } Guinea pig & Priming antigen & Bovine Tg & Mouse Tg \\
\hline \multirow{3}{*}{ CBA nnouse } & Bovine Tg & 55 & 0.4 \\
& Mouse Tg & 3.1 & 57 \\
\cline { 2 - 4 } & Bovine Tg & 18 & 2 \\
& Mouse Tg & 1.7 & 3 \\
\hline
\end{tabular}

Note: Cross-reaction of the proliferating response of spleen lymphocytes fram mice or guinea plgs immunised with bovine or mouse thyroglobulin (Tg) and stimulated in vitro with the same antigen. Note that $\mathrm{T}$ cells of mice primed with mouse $\mathrm{Tg}$ are tolerant for self-Tg.

Table 3. AChR-specific proliferation of immune lymph node cells

\begin{tabular}{cccc}
\hline & \multicolumn{3}{c}{ Simulation index } \\
\cline { 2 - 4 } Priming antigen & Torpedo AChR & Fetal bovine AChR & Rat AChR \\
\hline Torpedo AChR & 229 & 24 & 23 \\
Fetal bovine AChR & 5 & 100 & ND \\
Rat AChR & 1 & 20 & 20 \\
\hline
\end{tabular}

Note: Cross-reaction of the prollferating response of lymph node cells from rats immunised with Torpedo, fetal bovine or rat AChR and stimulated with Torpedo, fetal bovine or rat AChR. After stimulation with syngeneic AChR, T cells are prollferating, suggesting an absence of tolerance for rat AChR [modified from (De Bieets et al., 1982)].

Thus, in MG, a small amount of $A C h R$ or cross-reacting antigen is sufficient to start the immune response. When antibodies reach the endplates, the local debris of AChR antibody complexes is removed to the draining lymph nodes where the autoimmune tesponse is maintained. The muscle cell upregulates the AChR synthesis (Asher et al., 1993) and this ensures a continuous supply of self-antigen. Immune complexes containing $A C h R$ and anti-AChR antibodies are very efficient in stimulating the immune response (Melms et al., 1993).

T cells can be divided into $T$ helper-one (TH1) and Thelper-two cells (TH2) depending upon their cytokine profile. In EAMG mice, the pathogenic antibody response is TH1-dependent. In mice, manipulation of the balance in favour of TH2 cells protects against EAMG (Moiola et $\mathrm{al}_{\text {, }}$ 1998), In rats, however, both TH1 and TH2 cells are able to induce pathogenic anti-AChR antibodies. Manipulation of the TH1/ TH2 balance does not effect the severity of disease (Saoudi et al., 1999). The different requirements for $\mathrm{TH} 1$-dependent responses in rats and mice can best be explained by differences in complement fixation and/or activation by antibody subclasses in rats 
and mice. Indeed, unlike mouse IgG subclasses, both TH1- and TH2-associated rat Ig subclasses are capable of binding complement. In humans, both $\mathrm{TH} 1$ and $\mathrm{TH} 2$ cells are able to induce the production of complement-binding antibodies (Saoudi et al., 2000). It can be concluded from these studies that rat EAMG models are more relevant for the study of $M G_{\text {. }}$

\section{Anti-AChR antibodies}

The majority of anti-AChR antibodies in MG and EAMG (rat) are directed to the MIR in the a subunit. Apparently in contrast to the restricted specificity of anti-AChR antibodies, the origin of serum (Bionda et al., 1984; Lefvert and Bergstrom, 1978; Thompson and Krolick, 1992) and endplate antibody is polyclonal, indicating that a large population of plasma cells produce anti-AChR antibodies. Sequence analysis of monoclonal antibodies immortalised from $\mathrm{B}$ cells of EAMG animals reveals a high number of somatic mutations. These findings indicate that the immune response is antigen-driven and not the result of some accidental mutation. Among pathogenic antibodies, a high sequence homology ( $>90 \%$ ) has been found, but none of these antibodies were identical (Graus and De Baets, 1994).

To investigate the individual contributions of anti-human $A C h R$ responses in $M G$, the properties of monoclonal human antibodies were analysed. In total, 18 Fabs were isolated by three different groups. The 8 published amino acid sequences were all different (Farrar et al,, 1997; Graus et al., 1997; Rey et al., 2000). From the published sequences for antibodies from EAMG animals and MG patients, it can be concluded that pathogenic antibodies are not structurally related. Four human monoclonal antibodies directed to the AChR were cloned using a phage-display technique to isolate the anti-AChR immunoglobulin genes from B cells in the thymus of myasthenic patients. The antibodies were able to inhibit the binding of anti MIR antibodies and human MG sera to human $A C h R$ up to $80 \%$. They were also able to protect against $A C h R$ loss by antigenic modulation induced by MG sera, making them potential therapeutic tools for patients with a myasthenic crisis. All anti-MIR antibodies that recognize a small decapepticle $\alpha 67-76$ (Tzartos et al., 1998) at the top of the external side of a subunits. are structurally different. Indeed, antibodies in general recognize pentapeptides, which means that they can recognize at least 5 partially overlapping epitopes by their linear amino acid structure. In addition, the MIR contains conformational epitopes that can be recognised by their respective antibodies. In the pathogenesis of myasthenia, the rolle of antibodies against non-MIR epitopes, (i.e. directed to other subunits and cytoplasmic domains) has not been proven since only monoclonal antibodies against the MIR are capable of inducing EAMG. 


\section{Regulatory mechanisms at the endplate}

In $M G$ and $E A M G$, the immunological attack is directed against the postsynaptic membrane of the neuromuscular junction, where four regulatory mechanisms aim to limit or restore the damage.

\section{Role for complement and complement regulatory proteins}

Muscle cells are able to upregulate the synthesis of complement regulatory proteins (CD55, CD59, and vitronectin) as a defensive mechanism against complement-mediated damage (Hoedemaekers et al., 1997b). When anti-AChR antibodies bind to the tip of the postsynaptic folds, the classical complement pathway is activated. This results in the formation of the membrane attack complex (MAC), effecting focal lysis of the postsynaptic membrane. Complement-mediated focal lysis of the postsynaptic membrane also removes the cytoskeletal restraints on the $\mathrm{AChR}$ and facilitates internalisation and degradation.

In reaction to this process, decay-accelerating factor (DAF, CD55) inhibits $C 3$ production, and CD59 inhibits the formation of MAC. Both CD55 and CD59 are produced by muscle fibre specifically at the postsynaptic membrane. DAF deficient mice are subject to more severe EAMG than wild-type mice (Kaminski et al., 2003). Complement-deficient animals are protected against EAMG (Christadoss, 1989; Lennon et al., 1978). Rats with increased complement levels induced by the weakly virilising anabolic steroid nandrolone showed more severe signs of passive transfer EAMG and higher loss of AChR compared to untreated EAMG animals (De Baets et al., 1988).

In view of the important role of complement in the disease and the beneficial effect of complement inhibitors in EAMG, these inhibitors or noncomplement-fixing anti-MIR antibodies could be used as therapeutic agents to rapidly reverse a myasthenic crisis.

\section{Role of macrophages in EAMG}

In response to the antibody-mediated attack at the neuromuscular junction and the release of complement components, including $\mathrm{C} 3 \mathrm{a}$ and $\mathrm{C} 5 \mathrm{a}$, macrophages migrate to the endplate region. The question is whether these macrophages are a primary cause of $A C h R$ loss in $\mathbb{E A M G .}$ This question could be answered by depletion of macrophages in rats using irradiation. After induction of EAMG with anti-MIR monoclonal antibody 35, muscular weakness developed to a similar extent in macrophage-depleted animals as in control animals (Hoedemaekers et al., 1997a). AChR and complement depositions were similar in both groups. However, no infiltrating macrophages were seen in irradiated animals. Thus macrophage depletion does. not interfere with the induction of acute EAMG by injection of monoclonal antibody 35 . The macrophage infiltration seen in the nondepleted animals may be secondary to the focal dam- 
age at the endplate. In $M G$, macrophage infiltrations are present in muscle to a lesser extent, but mostly not in the vicinity of endplates. None of the inflammatory cells penetrate the muscle fibre near the endplate. In contrast, Maselli and colleagues (Richman et al., 1998) observed some macrophages in the endplate region. Based on the results of EAMG studies, however, it seems unlikely that macrophages play a role in endplate damage because endplate-bound anti-AChR antibodies and complement deposition suffice to induce AChR loss.

\section{AChR neosynthesis}

Induction of passive transfer EAMG results in a decrease of membrane-bound AChR levels and compensatory increase of $A C h R$ mRNA levels of all AChR subunits, as well as mRNA specific for myogenic transcription factors (Asher et al., 1993; Hoedemaekers et al., 1998), including myogenin and MRF (Balass et al., 1993). In very severe $E A M G$, a strong synaptic decrease of $a$ and $\varepsilon$ AChR mRNA occurs (Hoedemaekers et al., 1998).

The maintenance of high numbers of $\mathrm{AChR}$ at the endplate requires positive signals, including neuregulins (Sandrock et al., 1997). Destruction of the endplates in EAMG may interfere with positive signalling and may subsequently decrease AChR mRNA transcription. After the damage, postsynaptic membrane is restored and new AChRs are synthesised and inserted in the membrane.

\section{Role of AChR anchor proteins}

AChR accumulates at the top of postsynaptic folds at very high densities. Several proteins of the basal lamina and the postsynaptic skeleton are involved in maintaining AChRs at high density at the synapse (Figure 1). The synaptic basal lamina contains several synapse-specific molecules, including agrin and s-laminin. The postsynaptic cytoskeleton also contains $\mathrm{AChR}$-anchoring proteins such as rapsyn and utrophin. Mice knocked out for anchor proteins have abnormalities in the development of the neuromuscular junction, the number of AChRs, or their clustering (Gautam et al., 1999). In EAMG, the amount of rapsyn is decreased and the synthesis increased (Asher et al., 1994). Aged BN rats are resistant to both PT EAMG and EAMG induced by AI. The injection of mAb 35 (rat IgG1 complement fixing) does not induce muscular weakness nor clinical signs of EAMG in aged BN rats (Graus et al., 1993). This resistance could not be explained by deficient antibody uptake, increased antibody clearance, inaccessibility of AChR for antibodies in aged animals, nor deficient complement activation or increased complement regulatory proteins (Hoedemaekers et al., 1997d). The AChR degradation rates in old animals injected with $\mathrm{mAb} 35$ were comparable to those of age-matched control animals. This resistance of the AChR molecules to antibody-mediated degradation most likely resides at the level of the postsynaptic membrane. Semiquantitative measurement of several proteins of the postsynaptic membrane in aged rats revealed an increase in s-laminin and rapsyn. These experiments in aged animals suggest that the susceptibility and clinical course of EAMG and possibly MG is determined not only by the level of the immune attack, but also by the rigidity and the number of $\mathrm{AChR}$ anchor proteins in the postsynaptic membrane (Hoedemaekers et al., 1998). 


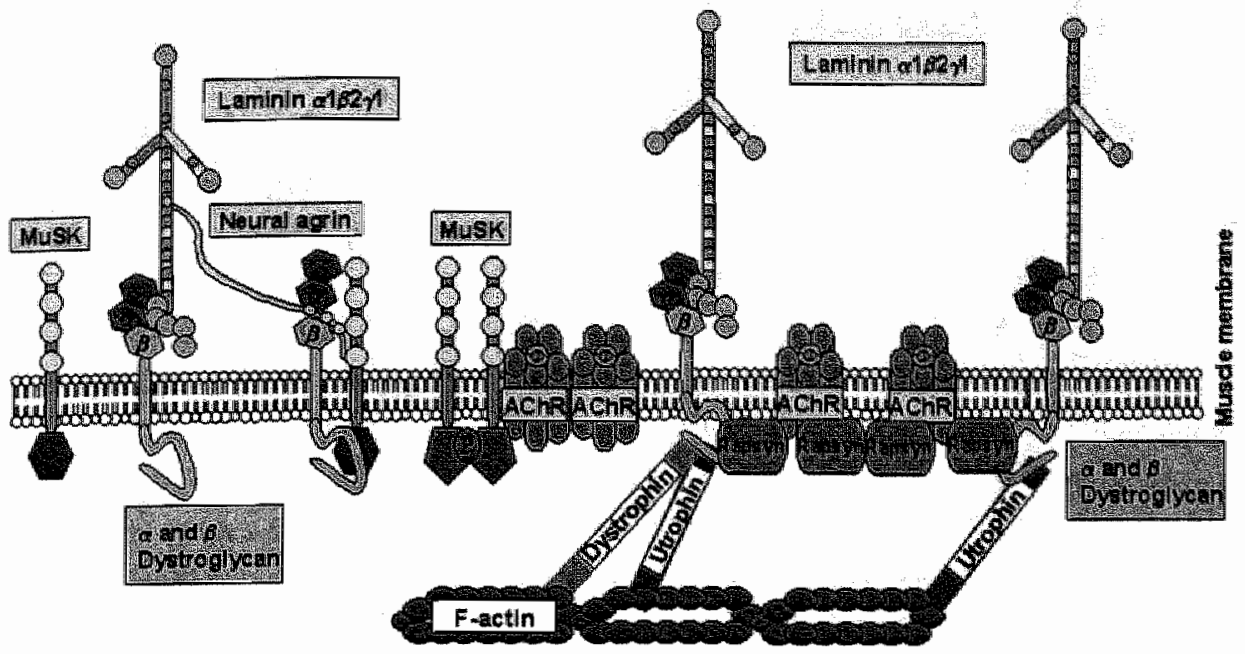

Figure 1. AChR clustering in the postsynaptic membrane. During neuromuscular synaptogenesis, neurally released agrin induces aggregation and tyrosine phosphorylation of acetylcholine receptors (AChRs) by acting through both the receptor tyrosine kinase MuSK (muscle-specific kinase) and the AChR-associated protein, rapsyn. Rapsyn, in turn, is linked to the cytoskeleton via $\beta$-dystroglycan, utrophin and F-actin. Moreover $\beta$ dystroglycan is linked to the basal membrane via a-dystroglycan and s-laminin (a1 $\beta 2 \gamma 1$ ).

\section{Acknowledgements}

We thank the Prinses Beatrix Fonds and LAssociation Française contre les Myopathies.

\section{References}

Asher $\mathrm{O}$, Fuchs $\mathrm{S}$, Souroujion MC. Acetylcholine receptor and myogenic factor gene expression in Torpedo embryonic development. Neuroreport Neuroreport 1994; 5; 1581-1.584.

Asher $O$, Kues WA, Witzemann V, Tzartos SJ, Fuchs S, Souroujon MC. Increased gene expression of acetylcholine receptor and myogenic factors in passively transferred experimental autoimmune myasthenia gravis. I Immunol $1993 ; 151: 6442-50$.

Balass M, Heldman X, Cabilly S, Givol D, Katchallski-Katzir E, Fuchs S. Identification of a hexapeptide that minacs a conformation-dependent binding site of acetylchaline receptor by use of a phage-epitope library. Proc Natl Acad Sci U S A 1993; 90: 10638-42.

Bionda A, De Baets MH, Trartos SI, Lindstrom IM, Weigle WO, Theophilopoulos AN. Spectrotypic analysis of antibodies to acetylcholine receptors in experimental autoimmune myasthenia gravis. Clin Exp Immunol 1984: 57: 41-50.

Christadoss P. Immunogenetics of experimental autoimmune myasthenia gravis. Crit Rev Immunol 1989; 9:247-78.

Christadoss $P$, Poussin $M$, Deng C. Animal models of myasthenia gravis. Clin Immunol 2000; 94: 75-87.

Damoiseaux IGGMC. Cyclosporin A-Induced autoimmunity in the ratcentral wersus peripheral tolerance. International Journal of immunopathology and pharmacology 2002; 15:81-87. 
De Bats MH Einarson $\mathrm{B}$, Lifdstrom IM, Weigle WO. Lymphocyte activation in experimental autoimmune myastherua gravis. IImmunol 1982;128:2228-35.

De Baets MH, Jansens $\mathrm{AM}$; $\mathbb{R}$ omball $\mathrm{CG}$, Weigle WO. A radioimmunoassay for murine thyroglobulin in serim: age-rejated increwse of serum thyroglobulin levels in ARRJ mice. Endocrinology 1983; 112: 1788-95.

De Bres MH, Verschumen , Daha MR, van Breda Vriesman Py. Effects of the rate of acetylcholime receptor synthesis on the severty of experimental autommune myasthena gravis. Immunol Res 1988; $7: 200-11$.

Parrar J, Portolanos, Whllcox W, Vincent $A$, Jacobson L. Newsom-Davis J, et al. Diverse Fab specific for acetylcholine receptorepitopes fiom a myasthenia granis thymus combinatorial library Int Immunol 1997; 9; $1311-8$.

Gautam M, DeChiara TM, Glass DF, Yancopoulos GD, Sanes JR. Distinct phenotypes of mutant mice lacking: agrim, MusK, or rapsyn. Brain Res Dew Brain Res 1999; 114: 171-8.

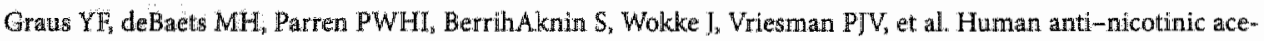
tylcholine receptor recombinant Fab fragments isolated from thymus-derived phage display libraries from Myasthenia grawis patients reflect predominant specificities in serum and block the action of pathogenic serum antibodies. Jimmunol 1997; 158: 1919-1929.

Graus YM, De Baets MH. Molecular and structural characterization of anti-acetylcholine receptor antibodies in experimental autoinmune myasthenia gravis. Adv Neuroimmunol 1994; 4: 457-74.

Graus MMF, Verschuuren JJGM, Spaans F, Jennekens F, Vriesman PJCV, Debaets MH. Age-Related Resistance to Experimental Autoimnune Myasthenia Gravis in Rats. J Immunol Journal of Immunology 1993; 150 : $4093 \times-4103$.

Gu D, Wogensen L, Calcutt NA, Xia C, Zhu S, Merlie JP, et al. Myasthenia gravis-like syndrome induced by expression of interferon gamma in the neuromuscular junction. J Exp Med 1995; 181: 547-57.

Hoedemaekers A, Bessereau IL, Graus Y, Guyon T, Changeux JP, Berrih Aknin S, et al. Role of the target organ in determining susceptibility to experimental autoimmune myasthenia gravis [In Process Citation]. J Neuroimmunal 1998; 89: 13.1. -41 .

Hoedemaekers A, Graus Y, Beijleveld I., wan Breda Vriesmam P, De Baets M. Macrophage infiltration at the neuromuscular junction does not contribute to $\mathrm{AChR}$ loss and age-related resistance to EAMG. I Neuroimmund $1997 \mathrm{a} ; 75: 147-55$.

Hodenackers $A$, Graus $Y$, wan Breda Vriesman $P$, de Baets M. Age- and sex-related resistance to chronic experinuental autoimmune myasthenia gravis (EAMG) in Brown Norway rats. Clin Exp Immunol 1997b; 107: 189-97.

Hoedemaekers $\mathrm{AC}_{\text {, van }}$ Breda Vriesman PI, De Baets $\mathrm{MH}$. Myasthen ia gravis as a prototype autoimmune receptor disease. Immunol Ples 1997c: 16:341-54.

Hoedemaekers AC, Verschun ren II, Spans E, Graus YF, Riemersma S, van Breda Vriesman PI, et al Age-retated susceptibility to experimental autoimmune myasthenia gravis: immunological and electrophysiological aspects. Muscle Nerve: 1997d; 20: 1091-101.

Kaminski $H]$, LI Z, Richmonds $C_{2}$ Ruff RL, Kusner $\mathbb{L}$. Susceptibility of ocular tissues to autoimmune diseases. Ann N Y Acad Sci 2003; 998:362-74.

Lefvert AK, Bergstrom K. Acetylcholine receptor antibody in myasthenia gravis: purification and characteriza.tion. Sand I lmmunol 1978;8:525-33.

Lennon WA, Saybold ME, Lindstrom JM, Cochrane C, Ulevitch $\mathbb{R}$. Role of complement in the pathogenesis of experimental antoimmune myasthenia gravis. J Exp Med 1978; 147: 973-83. 
Lindstrom JM. Acetycholine receptors and nyyasthenia. Muscle Nerwe $2000,23: 453-77$.

Melms A, Weissert R. Klinkert WEF, Schake BCG, Tzartos S, Wekerle H. Spedfic Intmune Complemes Angnent Invitro Acetyllcholine Receptor-Specific T-Cell Proliferation. Neurology Neurology $1993,43: 583$-588.

Moiola $L$ Galbiat $F$, Martino $G$, Amadio $S$ Brambilla $F$, Comi $G$, al. M-12 is wnolved in the induction of experimental autoimmume myasthena gravis, an antibody-mediated disease In Process Cutation . Eur] Immunol 1998; 28: 2487-97.

Monfardini C, Milani M, Ostlie $N_{s}$ Wang W Karachunski PI, Okita DK, et al Adoptive protection fom experi mental myasthenia gravis with $\mathrm{T}$ cells from mice treated nasally with acetylcholine receptor epitopes. I Neuroimmunol 2002; 123:123-34.

Patrick J, Lindstrom J. Autoimmune nesponse to acetyllholine receptor. Science 1973; 180:871 2 .

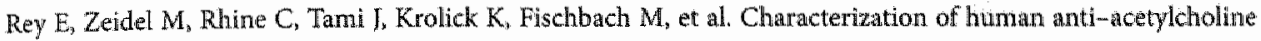
receptor monoclonal autoantibodies from the peripheral blood of a myasthenia gravis patient using com binatorial libraries. Chin Immunol 2000; 96: 269-79.

Richman DP, Agius MA, KIrvan CA, Gomez CM. Jairclough RH, Dupont BL, et al. Artibody effector mechanisms in myasthenia gravis. The complement hypothesis. Ann N Y Acad Sci 1998; 841:450-65.

Sandrock AW, Dryer SE, Rosen KM, Gozani SN, Kramer R, Theill LE, et al Maintenance of acetylcholine receptor number by neuregulins at the neuromuscular junction in wo. Science 1997; 276: 599-603.

Saoudi A, Bernard I, Houdemaekers A, Cautain B, Martinez K, Druet ., et al. Experimental autoimmtune myasthenia gravis may occur in the context of a polarized ThI- or Th2-type immune response in rats. I lmmunol $1999 ; 162 ; 7189-97$

Saoudi A, Guery JC, De Baets M. Is pathogenic humoral autoimmunity a 'Th1 response? Immunol Today 2000 ; 21: $306-7$.

Schonbeck $S$, Padberg 15, Hohlfeld $\mathrm{R}$, Wekerle $\mathrm{H}$. Transplantation of thymic autoimmune microenvironment to severe combined immunodeficiency mice. A new model of nyasthenia gravis. J Clin Invest 1992;90:245-50.

Shelton GD. Myssthenia grawis and disorders of neuromuscular transmission. Vet Clin North Am Small Anim Pract 2002; 32:189-206, vii.

Stassen MH, Meng F. Melgert E, Machiels BM, Im SH, Fuchs S, et all. Experimental atioimmune myasthenidg gravis in mice expressing human immunoglobulin loci. I Neurobmmunol 2003: 135: 56-61.

Thompson PA, Krolick KA. Acetylcholine receptor-reactive antibodies in experimental autoimmune myasthenia graws differing in disease-causing potential: subsetting of serum antibodies by preparative isoelectric focusing. Clin Immunol Immunopathol 1992; 62; 199-209.

Tzartos SI, Barkas T, Cung MT, Mamalaki A, Marraud M, Orlewski P, et al. Anatomy of the antigenic structure of a large membrane autoantigen, the muscle-type nicotinic acetylcholine receptor [In Process Citation]. Immunol Rev $1998 ; 163: 89-120$.

Weigle $\mathrm{WO}$. Analysis of autoimmunity through experimental models of thyroiditis and allergic encephalomyelitus. Adv Immunol 1980; 30:159-273.

Yang $\mathrm{H}_{3}$, Goluszko E, David C, Okita DK, Conti-Fine B, Chan 'TS, et al. Mapping myasthenia gravis-associated 'T' cell epitopes on human acetylcholine receptors in HLA trantsgenic mice. I Clin Invest 2002; 109: 1111-20. 


\section{Chapter 2}

\section{Increased Expression of Rapsyn in Muscles Prevents Acetylcholine Receptor Loss}

in Experimental Autoimmune Myasthenia Gravis

Mario Losen, Maurice H.W. Stassen, Pilar Martínez-Martinez,

Barbie M. Machiels, Hans Duimel, Peter Frederik, Henk Veldman, John H.J. Wokke, Frank Spaans, Angela Vincent and Marc H. De Baets

Brain, 2005; 128:23.27-37 


\section{Abstract}

Myasthenia grawis (MG) is usually caused by autoantibodies to the acetylcholine receptor (AChR). The AChR is clustered and anchored in the postsynaptic membrane of the neuromuscular junction by a cytoplasmic protein called rapsyn. We previously showed that resistance to experimental autoimmune $M G(E A M G)$ in aged rats correlates with increased rapsyn concentration at the neuromuscular junction (NM]). It is possible, therefore, that endogenous rapsyn expression may be an important determinant of $A C h \mathbb{R}$ loss and neuromuscular transmission failure in the human disease, and that upregulation of rapsyn expression could be used therapeutically. To examine first a potential therapeutic application of rapsyn upregulation, we induced acute EAMG in young rats by passive transfer of $A C h R$ antibody, mAb 35, and used in vivo electroporation to over-express rapsyn unilaterally in one tibialis anterior. We looked at the compound muscle action potentials in the tibialis an* terior, at rapsyn and $A C h R$ expression by quantitative radioimmunoassay and immunofluorescence, and at the morphology of the NMJs, comparing between the electroporated and untreated muscles, as wrell as between control and EAMG rats. In control rats transfected muscle fibres had extrasynaptic rapsyn aggregates, as well as slightly increased rapsyn and ACh $\mathbb{R}$ concentrations at the NMJ. In EAMG rats, despite deposits of the membrane attack complex, the rapsyn-overexpressing muscles showed no decrement in the compound muscle action potentials, no loss of $A C h R$, and the majority had normal postsynaptic folds, whereas endplates of untreated muscles showed typical AChR loss and morphological damage. 'These data suggest not only that increasing rapsyn expression could be a potential treatment for selected muscles of MG patients, but also raise the possibility that individual differences in innate rapsyn expression could be a factor in determining disease severity.

\section{Introduction}

In myasthenia gravis ( $M G$ ), the muscle form of the nicotinic acetylcholine receptor ( $A C h R$ ) is the target for pathogenic autoantibodies in eight out of ten MG patients, and the postsynaptic membrane of the neuromuscular junction (NMJ) is the target organ. AChR antibodies cause loss of functional $\mathrm{AChR}$ at the $\mathrm{NMI}$, either by crosslinking the receptors leading to increased internalisation (antigenic modulation), by activation of complement leading to focal lysis and morphological damage to the postsynaptic membrane, or by inhibiting the AChR's ion channel function (reviewed by (De Baets and Stassen, 2002). Loss of functional $A C h R$ impairs neuromuscular transmission, resulting in skeletal muscle weakness.

The AChR is clustered at high density at the NMJ and rapsyn, a $43-\mathrm{kD}$ protein, is essential for forming and maintaining AChR clusters. The clustering of these two postsynaptic proteins is initiated by agrin, a neuronal synaptogenic protein which acts via the muscle specific kinase, MuSK (Glass et al., 1996). MuSK causes phosphorylation of both MuSK and AChR resulting in the clustering and anchoring of preassembled AChR-rapsyn complexes to the cytoskeleton (Moransard et al..,2003). Mutations in the human rapsyn gene (RAPSN) or its promoter can lead to congenital myasthenic syndromes (Banwell et 
al, 2004; Burke et al., 2003; Dunne and Maselli, 2003; Muller et al., 2004; Olno et al, 2002; Ohno et al., 2003). Mice deficient in rapsyn die shortly after birth because the postsynaptic specialisation of the NMJ has failed to develop (Gautam et al, 1995). Rapsyn metabolically stabilises the $A C h R$, as shown by cotransfection of rapsyn and AChR expression plasmids (Phillips et al., 1997; Wang et al, 1999), and reduces the antigenic modulation of AChRs induced by the AChR monoclonal antibody ( $\mathrm{MAb}$ ) - 35 in transfected fibroblasts (phillips et al., 1997).

Experimental autoimmune $M G$ (EAMG) is an animal model of the human disease, and can be induced by passive transfer of $A C h R$ mAbs, eg. mAb 35, as well as by active immunisation against $A C h R$. Antigenic modulation and complement-mediated focal lysis of the postsynaptic membrane are the main pathogenic mechanisms of EAMG. Passive transfer EAMG in the rat is characterised by a very reproducible course of the disease, with substantial loss of AChR and postsynaptic membrane (Engel et al, 1979; Lindstrom et al., 1976a). The maximum muscle weakness is observed $48-60$ hrs after antibody injection in all animals, but susceptibility to EAMG is strongly dependant on strain, sex and age (Hoedemaekers et al., 1997b; Hoedemaekers et al., 1997c). Aged Brown Norway (BN) rats, in contrast to young animals, are resistant to the induction of passive or chronic EAMG (Graus et al., 1993) and there is little muscle weakness or loss of $\mathrm{ACh} R$. This distinction between young and aged $\mathrm{BN}$ rats cannot be explained by differences in immune responses or compensatory mechanisms such as increased expression of AChR or complement modulatory proteins (Hoedemaekers et al., 1997a; Hoedemaekers et al., 1997b; Hoedemaekers et al., 1997c). However, rapsyn expression is increased in aged $\mathrm{BN}$ rats as compared to young $\mathrm{BN}$ rats, suggesting a role for rapsyn in reducing $A C h R$ loss at the NMT (Hoedemaekers et al., 1998).

We hypothesised, therefore, that increasing rapsyn expression in EAMG muscles might protect against antibody-mediated loss of AChRs and prevent clinical evidence of muscle weakness. We induced rapsyn overexpression by in wivo electroporation (electropermeabilisation), an efficient, non-viral technique (Mir et al., 1999) that is potentially suitable for in wivo use. We examined the effects on the passive transfer model of EAMG induced by $\mathrm{mAb} 35$.

\section{Methods}

Construction of expression plasmid. The mouse rapsyn cDNA (a kind gift of Dr Z.Z. Wang (Wang et al., 1999)), was cloned into the mammalian expression vector pcDNA1.1/Amp (Invitrogen, Breda, The Netherlands) using EcoRI restriction sites, resulting in the plasmid pcDNA-rapsyn. The pcDNA-rapsyn plasmid was prepared for electropermeabilisation with the Qiagen Midiprep Kit, according to the manufacturer's manual, and finally dissolved in $0.9 \% \mathrm{NaCl}$ at a concentration of $2 \mu \mathrm{g} \mathrm{DNA} / \mu \mathrm{l}$.

Animals. 11-week-old female Lewis rats were obtained from the Department of Experimental Animal Services, University of Maastricht, The Netherlands, with permission of the Committee on Animal Welfare (DEC), according to Dutch governmental rules. 
Electropemeabilisation. Rats were anaesthetised by breathing halothane (5\% induction, $2 \%$ maintenance) in $\mathrm{NO}_{2} / \mathrm{O}_{2}(70 \% / 30 \%)$ supplied over a cylindrical ventilation cap held over the head. The hind legs were shaved. One tibialis anterior was injected with $75 \mu$ pcDNA-rapsyn $(2 \mu \mathrm{g} / \mu \mathrm{l})$ dissolved in $0.9 \% \mathrm{NaCl}$ and the other with $75 \mu 10.9 \% \mathrm{NaCl}$. The volume was injected in aliquots at 5-6 sites equally spread over the muscle. 5 min after the injection, the leg was placed between caliper electrodes (BTX, San Diego, CA, USA) with conductive gel. 8 pulses of $200 \mathrm{~V} / \mathrm{cm}, 20 \mathrm{~ms}, 1 \mathrm{~Hz}$ (reversal of polarity after first 4 pulses) were given (Electro Square Porator ECM 830, BTX, San Diego, CA, USA) (Mir et al., 1999).

Induction of EAMG. 14 days after electropermeabilisation with pcDNA-rapsyn rats were diwided into two groups: Half of the animals received no further treatment (controls) the other half was injected intraperitoneally with 20 pmol mAb 35 per $100 \mathrm{~g}$ body weight (Hoedemaekers et al, 1997a). After another 2 days, the rats were clinically scored, anesthetised for EMG and sacrificed. Clinical scoring and the EMG measurements were performed by blinded investigators.

Clinical scoring, The severity of clinical signs of disease in EAMG was scored by measuring muscular weakness. "The animals' muscle strength was assessed by their ability to grasp and lift repeatedly a $300 \mathrm{~g}$ rack from the table, while suspended manually by the base of the tail for 30 seconds (Hoedemaekers et al., 1997b; Hoedemaekers et al., 1997c; Verschuuren et al., 1990). Clinical scoring was based on the presence of tremor, hunched posture, muscle strength and fatigability. Disease severity was expressed as: 0 , no obvious abnormalities; + , no abnormalities before testing, but reduced strength at the end; ++ , clinical signs present before testing, i.e. tremor, head down, hunched posture, weak grip; +++ , severe cininical signs present before testing, no grip, moribund (Lennon et al., 1975).

Electromyography (EMG). Decrement of compound muscle action potential (CMAP) was measured in the tibialis anterior muscles of 5 EAMG rats 48 hours after $\mathrm{mAb} 35$ transfer. Rats were anesthetised with $60 \mathrm{mg} / \mathrm{kg}$ sodium pentobarbital. For stimulation, two small monopolar needle electrodes were used. The cathode was inserted near the peroneal nerve at the level of the knee and the anode more proximal and lateral at a distance of 3 to $4 \mathrm{~mm}$. For recording, a third monopolar needle electrode was inserted subcutaneously over the tibialis anterior muscle. A ring electrode distally around the relevant hind leg served as a reference and the animal was grounded by a ring electrode around the tail. Stimulation and recording were performed with the EMG system Viking IV (Nicolet Biomedical Inc., Madison, WI, USA). To detect a decrementing response, series of 8 supramaximal stimuli were given at $3 \mathrm{~Hz}$. Stimulus duration was $0.2 \mathrm{~ms}$. The test was considered positive for decrement when both the amplitude and the area of the negative peak of the CMAP showed a decrease of at least $10 \%$ (Kimura, 2001). To demonstrate reproducibility, at least 3 recordings were made of all investigated muscles. During the measurements, skin temperature was kept between $35^{\circ} \mathrm{C}$ and $37^{\circ} \mathrm{C}$ by means of an infrared heating lamp (DISA, Copenhagen, Denmark). All EMG studies were performed by the same investigator (F.S.).

Immunohistochemical staining. Isolated tibialis anterior muscles of 4 control and 4 EAMG animals were frozen in melting isopentane. Cryosections of $10 \mu \mathrm{m}$ were dried, fixed 
in acetone at $4^{\circ} \mathrm{C}$ for $10 \mathrm{~min}$ and blocked for $20 \mathrm{~min}$ with PBSA (phosphate-buffered saline with $2 \%$ bovine serum albumin). Sections were incubated with mouse anti-rapsyn mAb 1234 (1/1000 in PBSA; (Bloch and Froehner, 1987); a kind gift of Prof. S.C. Froehner), with $\mathrm{mAb} 2 \mathrm{~A} 1$ against rat C5b-9 (membrane attack complex), (1/100 in PBSA; provided by W.G. Couser) or with rabbit anti-vesicular acetylcholine transporter (VAChT; 1.500; Phoenix Pharmaceuticals, Belmont, USA) for 45 min and subsequently washed with PBS with $0.05 \%$ Triton-X100. Subsequently the sections were incubated for 45 min with Alexa594-conjugat* ed a-bungarotoxin (Alexa594-a-BT; diluted 1/300 in PBSA; Molecular Probes, Leiden, The Netherlands) and the corresponding secondary antibody: biotinylated donkey anti-mouse Ig (1/200; minimal cross-reaction with rat IgG; Jackson Immunoresearch, West Groove, USA) or Alexa350-conjugated goat anti-rabbit (1/200; Jackson Immunoresearch). After washing as described above, the biotinylated antibodies were stained with luorescein-streptavidin (1/300; Jackson Immunoresearch). Coverslips were mounted with $0.2 \mathrm{M}$ Tris $\mathrm{pH}=8$ with $80 \%$ (v/v) glycerol.

Quantitative immunofluorescence analysis. Pictures of muscle sections were taken using a Provis AX70 fluorescent microscope (Olympus, Hamburg, Germany) with a digital camera (U-CMAD-2, Olympus) and the AnalySIS software (Soft Imaging Systems, Münster, Germany). The exposure time was set to a constant value ensuring that no saturation of the pictures occurred. Also all other microscope settings were maintained constant. Sections triple stained for VAChT, rapsyn and $A C h R$ were photographed using filters for Alexa350, FITC and Alexa594 fluorescence. A five fold reduced concentration of primary antibodies and bungarotoxin did not result in a weaker staining, therefore they did not limit staining intensity. Pictures were analysed using the ImageJ software (version 1.32; http:// rsb.info.nih.gov/ij). Endplate areas were identified as regions of VAChT staining and the mean intensity of VAChT, rapsyn and AChR staining was measured in the corresponding area. The ratio of $A C h R: V A C h T$ and rapsyn:VAChT was calculated for more than 75 endplates as a relative measure for the synaptic $A C h \mathbb{R}$ and rapsyn concentration. All sections were stained and processed in parallel to avoid inter-assay variations. Differences between endplates in rapsyn-treated versus control samples were analysed using an unpaired t-test.

Two-photon laser scanning microscopy. High resolution pictures of endplates were taken using a two-photon laser scanning microscope setup as described by (van Zand. voort et al., 2004) with the following modifications. A $60 \mathrm{x}$ oil immersion objective with a numerical aperture of 1.0 was used. Further magnification was achieved by optical zoom (3x) of the scan head. Picture stacks with a axial resolution of $0.15 \mu \mathrm{m}$ and ateral resolu* tion of $0.13 \mu \mathrm{m}$ were taken using photomultipliers accepting wavelengths of $520-540 \mathrm{~nm}$ and over $560 \mathrm{~nm}$. To remove noise each image was filtered applying the Kalman filtering procedure on two subsequent images. For deconvolution, 3D reconstruction and projection of the images the Autodeblur and Imaris software (vers. 4.0.3, Bitplane AG, Zürich, Switzerland) was used.

Radioimmunoassay (RLA) for measurement of AChRs. The AChR concentrations of isolated tibialis anterior muscles from 5 control and 5 EAMG rats were measured as de. 
scribed previously (Lindstron et al, $1976 \mathrm{~b}$; Verschuuren et al., 1992) with the following modifications: Muscles were minced and homogenised with an Ultra-Turrax ( 3 times for 30 s at $4^{\circ} \mathrm{C}$ ) in $10 \mathrm{ml}$ Buffer A (PBS, $10 \mathrm{mM}$ EDTA, $10 \mathrm{mMNaN3}, 10 \mathrm{mM}$ iodoacetamide, $1 \mathrm{mM}$ PMSF). Homogenate was centrifuged $(22100 \mathrm{~g}, 30 \mathrm{~min})$ and the resulting pellet was resuspended in $2.5 \mathrm{ml}$ Buffer $\mathrm{B}$ (Buffer $\mathrm{A}$ with additional $0.5 \%$ Triton $\mathrm{X}-100$ ). Extraction was performed for $1 \mathrm{~h}$ at $4^{\circ} \mathrm{C}$ on a reciprocal shaker. After centrifugation $(22100 \mathrm{~g}$, $30 \mathrm{~min}), 6$ aliquots of 250 , w were taken from the supernatant and incubated with ${ }^{125} \mathrm{I}-\mathrm{a}-$ BT (74 TBq/mmol. Amersham Pharmacia Biotech Benelux, The Netherlands) $(12.5 \mu \mathrm{l} / \mathrm{ml})$ followed by rat polyclonal anti-rat $\mathrm{AChR}(150 \mu \mathrm{l} / \mathrm{ml})$. As a negative control, 3 of the aliquots were supplemented with $1 \mathrm{mM}$ acetylcholine and $1 \mathrm{mM}$ neostigmine bromide. After overnight incubation at $4^{\circ} \mathrm{C}$, the immune complexes were precipitated with goat anti-rat $\operatorname{Ig}(100 \mu \mathrm{l}$ polyclonal serum) for $4 \mathrm{~h}$ and centrifuged at $15000 \mathrm{~g}$ for 5 minutes. The pellets were washed 3 times in PBS with $0.5 \%$ Triton X-100 and measured in a $\gamma$-counter. Results are calculated in fmol \pm standard deviation and differences between concentrations are presented in percentage \pm standard error of the difference of the means. Differences between samples were analysed using a one-sided t-test (paired differences were used for comparison of rapsyn-treated versus control in the same experimental group).

Electron microscopy, 5 EAMG rats and 2 control rats with unilateral-rapsyn treatment in the tibialis anterior were anesthetised with $60 \mathrm{mg} / \mathrm{kg}$ sodium pentobarbital and transcardially perfused with Tyrode solution $(0.1 \mathrm{M})$ followed by fixation buffer $(2.5 \% \mathrm{glu}$ taraldehyde in $0.1 \mathrm{M}$ phosphate buffer $\mathrm{pH}=7.4$ ). The tibialis anterior muscles were removed, postfixed for $2 \mathrm{hrs}$ and sectioned on a vibratome 1000 at $1 \mathrm{~mm}$. Sections were postfixed with $1 \%$ osmiumtetroxide in 0.1 phosphate buffer, $\mathrm{pH} 7.4$, dehydrated through a graded ethanol series and embedded in epoxy resin (Glycid ether 100, Serva, Heidelberg, Germany). Endplates were located in toluidine blue-stained semi-thin sections from the central region of each muscle. Ultra thin sections from selected areas were contrasted with uranyl acetate and lead citrate and viewed with Philips CM 100 electron microscope. At least five endplate regions were photographed from each muscle. Pictures were scanned for morphometric analysis using the ImageI software. Analysed parameters included the size of nerve boutons and the length of the presynaptic and postsynaptic membrane (Engel ett al., 1979; Engel et al., 1976). Endplates with infiltrated mononuclear cells could not be analysed, but the number of such endplates were counted.

\section{Results}

\section{Effects of rapsyn overexpression on rapsyn and $A C h R$ distribution in healthy muscle}

To look first at the effects of overexpressing rapsyn in normal rat muscles, the left tibialis anterior muscle was electropermeabilised after pcDNA-rapsyn injection (rapsyn-treated muscle). As a control, the right tibialis anterior muscle was electropermeabilised after injection of saline or the empty vector (untreated muscle). After two weeks, the muscles were 
isolated and cryosections were double-stained for rapsyn, using monge amilody, and tor AChR using Alexa594-a-BT. Muscle fibres from untreated or treated muscess bad ACh and rapsyn restricted to the neuromuscular junctions, with co-localstion of $\mathrm{CHR}$ and

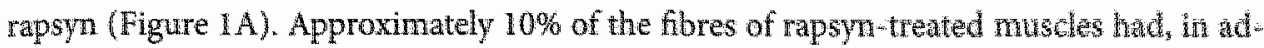
dition, extrasynaptic rapsyn aggregates. Most were small aggregates distributed over large

AChR

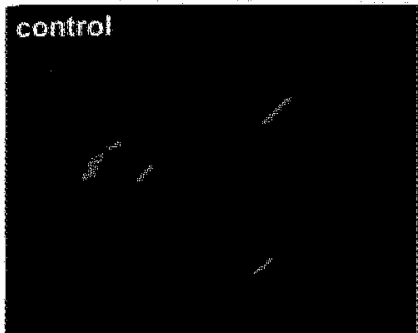

control trapsyn rapsyn
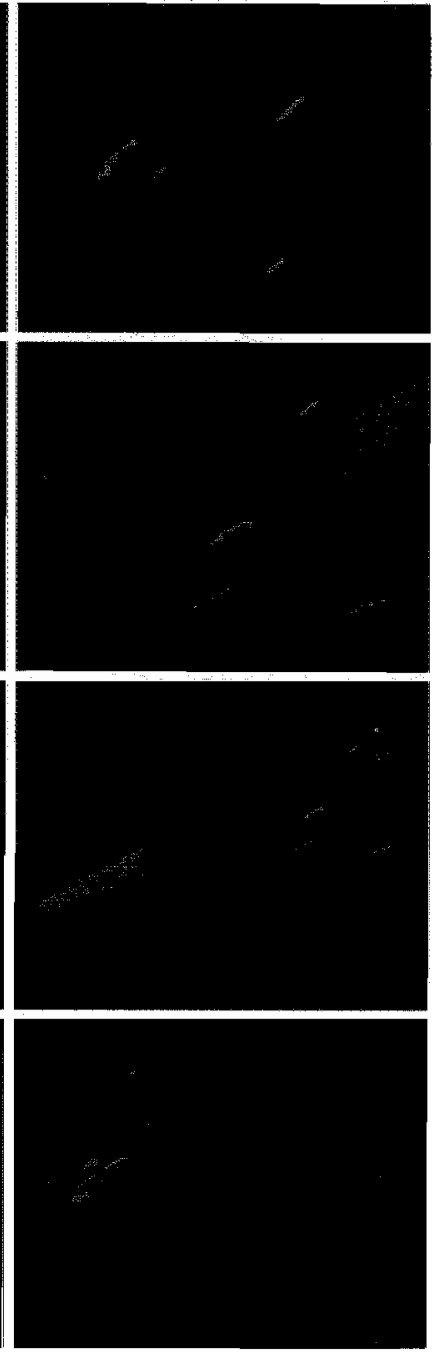

merg
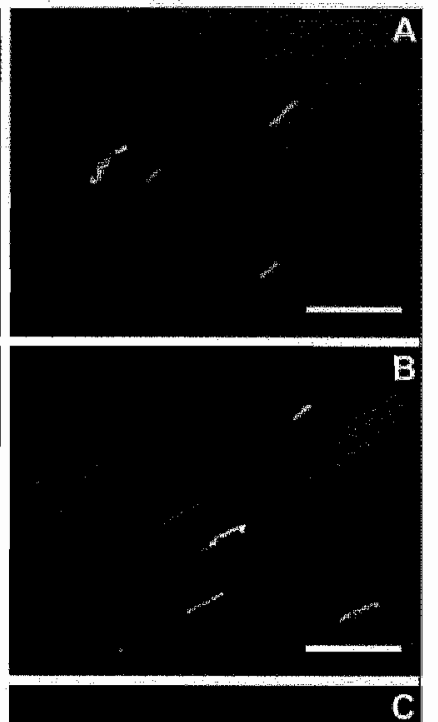

control + rapsyn

control + rapsyn

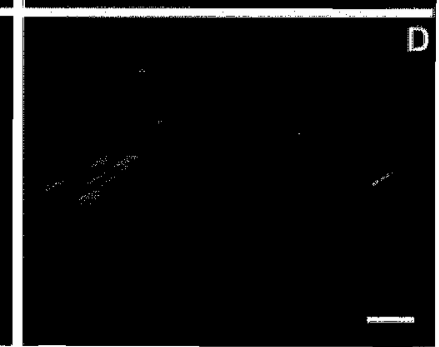

Figure 1. Immunohistochemical staining of control and rapsyn-4reated control muscles. Cryosectlons of muscles were double-stained with Alexa594-conjugated a-bunglarotoxin (left) and mouse anti-rapsyrin mAb1234 (middle); merge on the right. (A) AChR and rapsyn staining of untreated tibialis anterior muscle of a control rat. $(B, C, D)$ Rapsyn overexpression in control rats with an increased rapsyn concentration in the endplate and variable amounts of extrasynaptic rapsyn aggregates; which did not co-localise with AChR. Bars are 100 um; all pictures are taken under the same conditions. 
parts of the cytoplasm (Figure $1 B, C$ ), but some were large, heavily stained extrasynaptic aggregates (Figure 1D). AChR did not co-localise with any of the extrasynaptic rapsyn aggregates (Figure 1D).

To measure the $A C h R$ and rapsyn expression more quantitatively, we performed radioimmunoassay of muscle extracts labelled with ${ }^{125} \mathrm{I}-\mathrm{a}-\mathrm{BT}$, and quantitative immunofluorescence on the muscle fibres, using an antibody to the vesicular acetylcholine transporter (VAChT) as a standard. The results are shown in Figures $2 \mathrm{~A}, \mathrm{~B}, \mathrm{C}$ (control) and in Figure $2 \mathrm{D}$. Electroporation of the empty vector did not change the AChR concentration compared to untreated muscles (data not shown). Rapsyn-treated muscles showed significantly more $A C h R$ content by $\mathbb{R I A}(+42 \pm 15 \%, n=5 ; p=0.018$, paired $t$ test) than untreated muscles (Figure $2 \mathrm{~A}$ control). Quantitative analysis of the Alexa594- $a-B T$ staining showed a significantly increased amount of AChR per NM) (Figure 2B control; $+21436 \%, n=78, p<0.001$, unpaired $t$ test). Similarly, the relative amount of rapsyn was also increased (Figure $2 \mathrm{C}$ control; $+44 \pm 68 \%, n=78, \mathrm{p}<0.001$, unpaired t test) compared to untreated control muscle. When the relative amounts of $A C h R$ were plotted against the relative amounts of rapsyn (Figure $2 D$ ), there was a highly linear relationship in the untreated muscles $(r=0.81, p<0.001)$, but less so in the rapsyn-treated muscles $(r=0.58$, $\mathrm{p}<0.001$, not shown). The ratio of rapsyn to $A C h R$ was increased from $1: 1$ up to $2: 1$ in individual rapsyn treated fibres. The relationship between rapsyn and AChR in the rapsyn transfected muscles can be fitted to a saturation curve (Figure $2 \mathrm{D}$ dashed line; $r=0.79$ and $p<0.001$ ). These findings suggest that there was potential for increased expression of rapsyn at the NMJs in normal muscle, but a limitation as to how much the $\mathrm{AChR}$ concentration could increase at the same junctions.

\section{Effects of rapsyn overexpression on neuromuscular transmission and AChR numbers in EAMG rats}

Firstly, EAMG was induced in five rats with mAb 35 two weeks after electropermeabilisation of the tibialis anterior muscles. Within 48 hours all of these five, and all others used in this study (total number $=20$ ) had clear clinical signs of EAMG (score of ++ in each animal). As expected, the untreated tibialis-anterior muscles showed a decremental response of the CMAP, with a mean amplitude decrement of $19.6 \pm 5.7 \%(n=5)$ and a mean area decrement of $18.4 \pm 2.5 \%$, confirming a neuromuscular transmission defect. By contrast, the contralateral, rapsyn-treated, muscles of the same five animals showed no decrement $(-1 \pm 1 \%, n=5)$, indicating that rapsyn overexpression prevented the development of the neuromuscular junction defect in passive transfer EAMG.

To confrm that the electromyographic differences were associated with changes in AChR expression, the concentration of AChR was first measured in EAMG muscle by RIA (on a different set of five EAMG and five control rats). The results are shown in Figure $2 \mathrm{~A}$ where they can be compared with those from muscles of control rats. The $A C h R$ concentration in untreated muscles of EAMG rats was reduced by $39 \pm 10 \%$ compared to the untreated muscles of the control group (Figure $2 A ; 11 \pm 3 \mathrm{fmol} / \mathrm{g}$ compared 

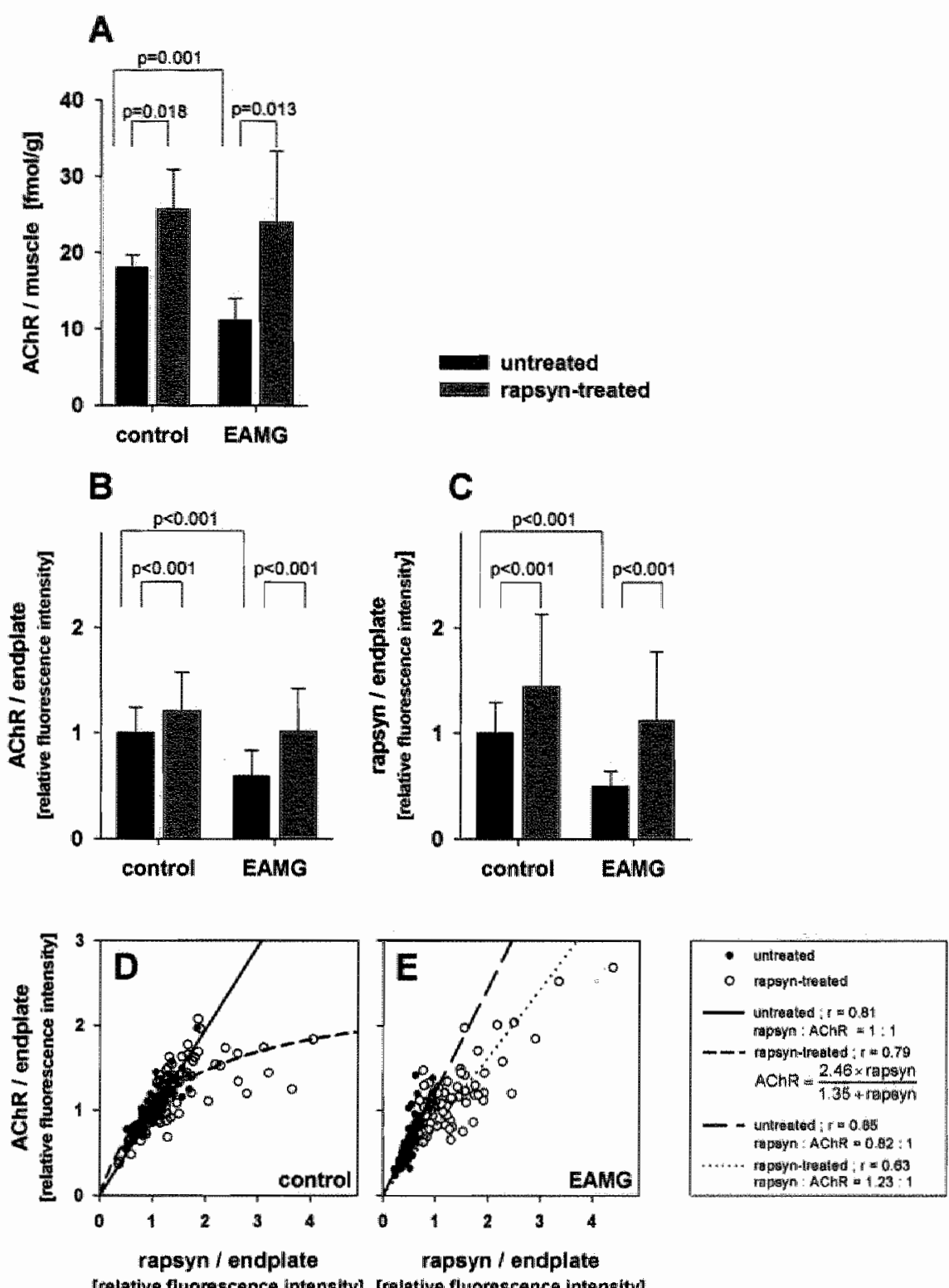

[relative fluorescence intensity] [retative fluorescence intensity]

Figure 2. Effect of rapsyn overexpression on AChR and rapsyn level in mAb 35-induced EAMG rats. The AChR concentration of untreated and rapsyn-treated tibialis anterior muscles was measured by using ${ }^{125}$ |labelled a-bunglarotoxin (A) and by quantifying a-BT-Alexa594 staining of endplates (B). In the same endplates rapsyn levels (C) were analysed by quantification of imimunohistochemical staining with anti-rapsym mAb 1234. Endplate areas were identified using a polyclonal antibody against the vesicular acetylcholine transporter (VAChT), and the intensities of a-BT-Alexa594 and rapsyn staining were normal ised against the intensity of this protein. The average AChR and rapsyn concentration of the untreated muscle of the control animals. was set to 1. The relative amount of AChR versus rapsyn in individual endplates are plotted in (D) for the control animals and in (E) for the EAMG animals. Closed symbols: endplates of untreated muscles; open symbols: endplates of rapsyn-treated muscles. In the rapsyn treated muscles a large proportion of endplates have an increased amount of rapsyn. 
to $18 \pm 2 \mathrm{fmol} / \mathrm{g} ; \mathrm{p}=0.001$, unpaired $\mathrm{t}$ test) but rapsyn-treated EAMG muscles had significantly higher AChR concentrations than the contralateral untreated muscles ( $24 \pm 9 \mathrm{fmol} /$ $\mathrm{g}$, compared with $11 \pm 3 \mathrm{fmol} / \mathrm{g}, \mathrm{p}=0.013$, paired $\mathrm{t}$ test). Indeed the rapsyn-treated muscles of EAMG rats had similar AChR concentrations to those of the rapsyn-treated control muscles $(26 \pm 5 \mathrm{gmol} / \mathrm{g}: \mathrm{p}=0.75$, unpaired $\mathrm{t}$-test).

To examine further the changes at the neuromuscular junction we performed immunofluorescence studies as above. The examples shown in Figure 3A, B can be compared to Figure $1 \mathrm{~A}, \mathrm{~B}$ that were prepared and examined under the same conditions. In EAMG rats, $A C h R$ and rapsyn staining of endplates of the untreated tibialis anterior muscle was weak (Figure $3 \mathrm{~A}$ ), whereas that in the rapsyn-treated contralateral tibialis anterior muscle (Figure 3B) was similar to that in untreated control muscles (compare Figure 1A). Interestingly, this effect of rapsyn-treatment was independent of visible rapsyn aggregates in the fibres (Figure 3B). The quantification of immunohistochemical staining of endplates in EAMG animals confirmed a reduction in the relative concentrations of AChR (by $41 \pm 17 \%, n=100, p<0.001$, unpaired $t$ test) and rapsyn (by $51 \pm 15 \%, n=100, p<0.001$ unpaired $t$ test) at the endplates of the untreated EAMG rats (Figure $2 \mathrm{~B}, \mathrm{C}$ ), and demonstrated that the rapsyn-treatment increased the relative $A C h R$ and rapsyn concentrations in EAMG muscle to levels similar to those in untreated control muscles (1.02 \pm 0.40 of $A C h R ; n=100, p=0.75$ and $1.12 \pm 0.65$ of rapsyn; $n=100 ; p=0.15$ ) although the wide standard deviations reflect the variability in $\mathrm{AChR}$ and rapsyn expression at individual NMJs. There was a highly linear correlation between the AChR concentrations by RIA (Figure 2A) and by quantitative immunohistochemistry (Figure 2B) in the four experimental groups $(r=0.93$ ).

We used two-photon microscopy, at higher resolution, to examine the NMIs in more detail. A representative endplate in an untreated EAMG muscle (Figure $3 \mathrm{C}$ ) shows a simplified postsynaptic membrane with reduced amounts of rapsyn and AChR. By contrast, the rapsyn-transfected fibre shows intense staining of rapsyn and AChR in a normal-appearing NMJ, with less intense rapsyn staining of the surrounding cytoplasm and extrasynaptic membrane (Figure 3D).

The plot of relative $A C h R$ concentration versus relative rapsyn concentration at individual endplates (Figure 2E, closed symbols) in untreated EAMG muscles shows that the relative values of each are lower in general, as expected from Figure $2 \mathrm{~B}$ and $\mathrm{C}$, and there is a highly linear correlation between $A C h R$ and rapsyn $(r=0.85, p<0.001)$ as also found in untreated control muscles (Figure 2D). But in sections of rapsyn-treated EAMG muscles (Figure 2E, open symbols) 64 out of $100(64 \%)$ endplates had a rapsyn concentration that was significantly higher than in the contralateral EAMG muscles (>mean + 2 SDs of untreated EAMG endplates), compared with only 6 out of $110(5 \%)$ of the endplates in the untreated muscle sections. Therefore, approximately $59 \%$ of endplates ( $64 \%$ minus $5 \%$ ) had increased expression of rapsyn. Similarly ${ }_{n} 40$ out of 100 endplates (40\%) had a significantly increased AChR concentration compared to only 6 out of $110(5 \%)$ in 
AChR
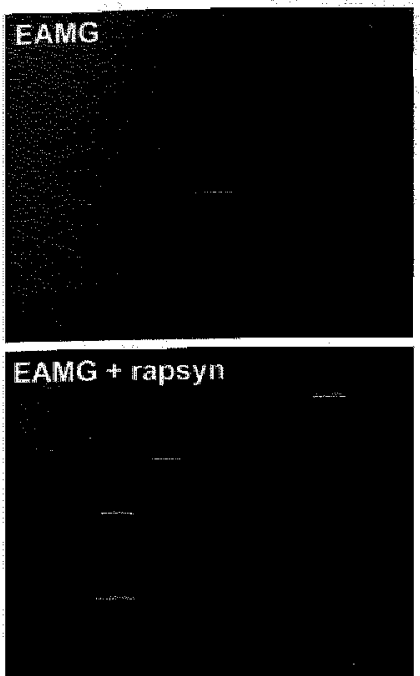

\section{EAMG}

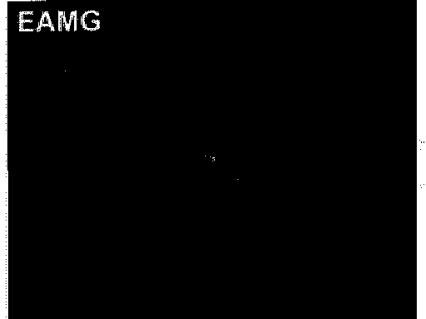

EAMG + rapsym

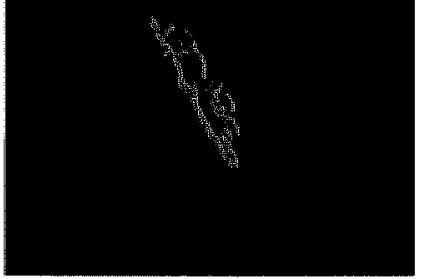

rapsyn
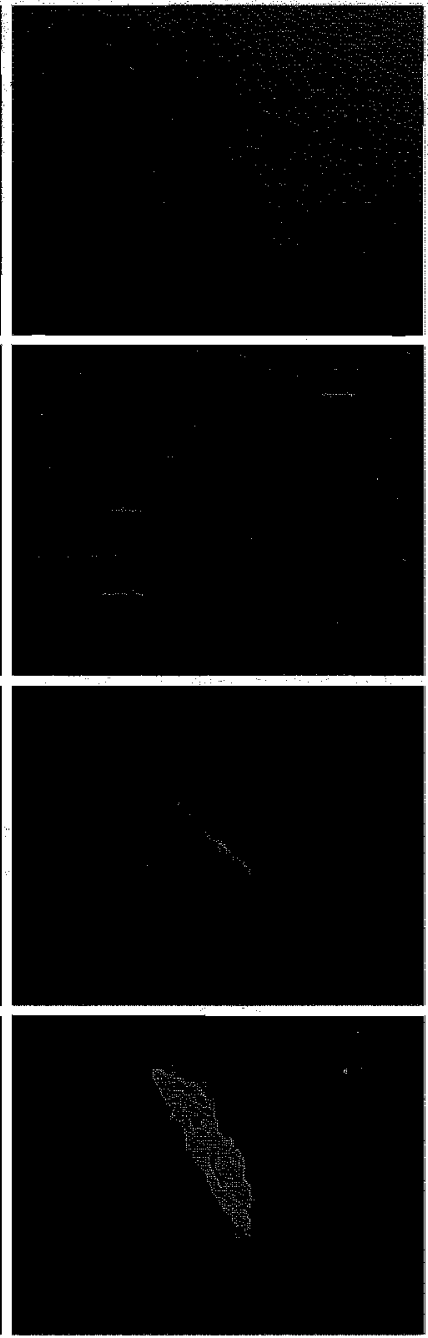

\section{merge:}
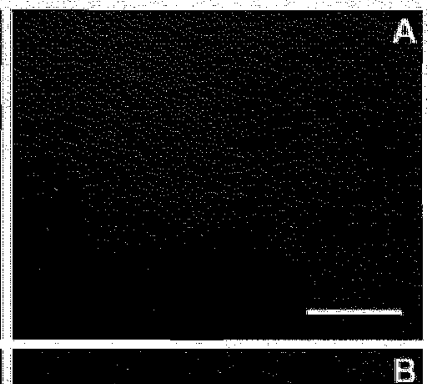

$\mathbf{B}$

Figure 3. Immunohistochemical analysis of rapsym expression in the NMJ of EAMG rats using standard fluorescence ( $A$ and $B$; scale bars are $100 \mu \mathrm{m}$ ) and two-photon laser scanning microscopy ( $C$ and $D$; scale bars are $20 \mu \mathrm{m}$ ). (A and C) Endplates of an untreated tibialis anterior muscle 2 days after induction of EAMG. Endplates showed reduced concentration or complete loss of $A C h R$ and rapsyn. (B and D). Rapsyn overexpression in the contralateral tibialis anterior muscle of the same EAMG animal. Rapsyn-treated endplates appeared very similar to those of rats without EAMG (Figure 1).

the contralateral untreated EAMG muscles. Since the relationship between rapsyn and AChR is linear $(0.82: 1 ; \mathrm{p}<0.001$; Figure $2 \mathrm{E}$, open symbols), and different from untreated EAMG muscles, it appears that the increased rapsyn expression has helped to protect about $35 \%$ of the NMIs ( $40 \%$ minus $5 \%$ ) from AChR loss. 


\section{Rapsyn overexpression does not prevent complement deposition at the neuromuscular junction}

In untreated muscles, mAb 35 reduced $A C h R$ expression and induced deposition of the membrane attack complex of complement (MAC) at the endplates (Figure 4A), consistent with a complement-dependent degradation of AChRs. Interestingly, in rapsyn-treated muscles, the rnembrane-attack complex was still present, but not associated with decreased AChRs (Figure $4 B$ ).

\section{Rapsyn Overexpression Prevented the Destruction of Postsynaptic Folds in EAMG Rats}

To see therefore, whether the rapsyn-treatment was associated with less destruction of the postsynaptic membrane in the treated EAMG muscles (as predicted from Figure 3D), we performed electronmicroscopic observations and morphometric analysis on 2 control and 5 EAMG rats. Rapsyn treatment did not appear to alter the structure of the neuromuscular junction in control muscles (Figure 5A, B and Table 1) which showed normal postsynaptic folds in 28/28 regions examined. In the untreated muscles of EAMG rats all endplates observed showed morphological abnormalities. At 28/46 endplates regions infiltrating cells were found (macrophages and neutrophils); others had reduced postsynaptic folds or even a complete loss of postsynaptic folding (Figure $5 \mathrm{C}$ ). The average length of the postsynaptic membrane was significantly reduced compared to normal endplates ( $<<0.0001$; Table 1). In the contralateral

\section{AChR}

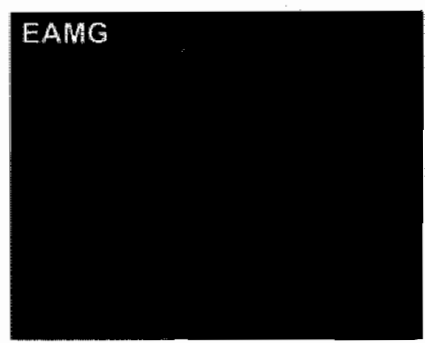

EAMG + rapsyn
MAC
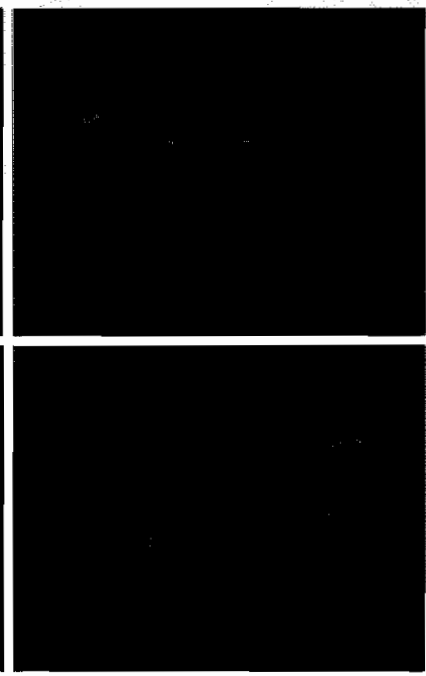

merge

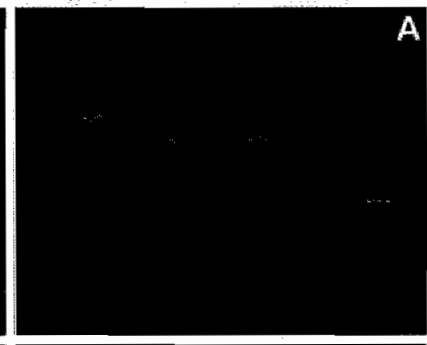

B

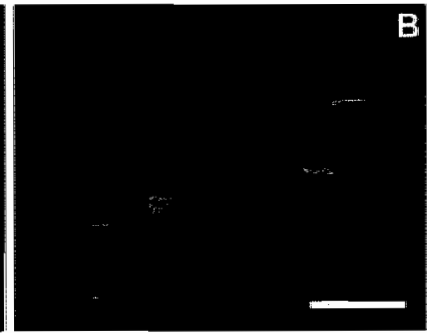

Figure 4. Immu mohistochemical staining of membrane attack complex (MAC) in the neuromuscular junctions in EAMG rats. Endpliates of untreated $(A)$ and rapsyn-treated muscles (B) were stained with $A$ lexa594-C-BT (left) and MAB 2All against rat C5D-9 (MAC, middle); merge on the right. The MAC staining of the untreated muscle reweals that endplates contained a very low concentration of AChR. MAC colocalised with the receptor in the endplates of the rapsyn-treated muscle. Scale bar is $100 \mu \mathrm{m}$. 
Table 1. Morphometric analysis of endplates

\begin{tabular}{|c|c|c|c|c|c|}
\hline & Regions Analysed" & $\begin{array}{l}\text { Nerve Boutoni } \\
\text { Area fum? }\end{array}$ & $\begin{array}{l}\text { Presyrapitic Wembrane } \\
\text { Length Lum] }\end{array}$ & $\begin{array}{l}\text { Postsynaptic Membrane } \\
\text { Length [um] }\end{array}$ & $\begin{array}{l}\text { Menbrane Length hato } \\
\text { Postsynaptic / Prosynaptic }\end{array}$ \\
\hline $\begin{array}{c}\text { untreated } \\
\text { control }\end{array}$ & 46 & $6.0 \pm 0.4$ & $8.2 \pm 0.6$ & $41.4 \pm 4.0$ & $5.3 \pm 0.4$ \\
\hline $\begin{array}{l}\text { nepsyn-treated } \\
\text { control }\end{array}$ & 28 & $7.9 \pm 1.2$ & $8.1 \pm 0.9$ & $39.6 \pm 4.4$ & $5.2 \pm 0.5$ \\
\hline $\begin{array}{l}\text { untreated } \\
\text { EAMG }\end{array}$ & $118^{\circ}$ & $7.3 \pm 1.2$ & $8.3 \pm 0.7$ & $16.8 \pm 3.7$ & $1.9 \pm 0.3^{i}$ \\
\hline $\begin{array}{c}\text { rapsyn-treated } \\
\text { EAMG }\end{array}$ & $46^{3}$ & $6.2 \pm 0.7$ & $7.1 \pm 0.5$ & $32.9 \pm 3.0$ & $5.2 \pm 0.6$ \\
\hline
\end{tabular}

Mean $\pm S E$

a region here refers to an area of one or two synaptic boutons

28 more regions could not be analysed because of infiltrating mononuclear cells.

significantly different from untreated control endplates $(p<0.0001)$ and significantly different from treated EAMG endplates ( $p<0.0001$ ) other differences in this table are not significant ( $p>0.05$ )

3 more regions could not be analysed because of infiltrating mononuclear cells
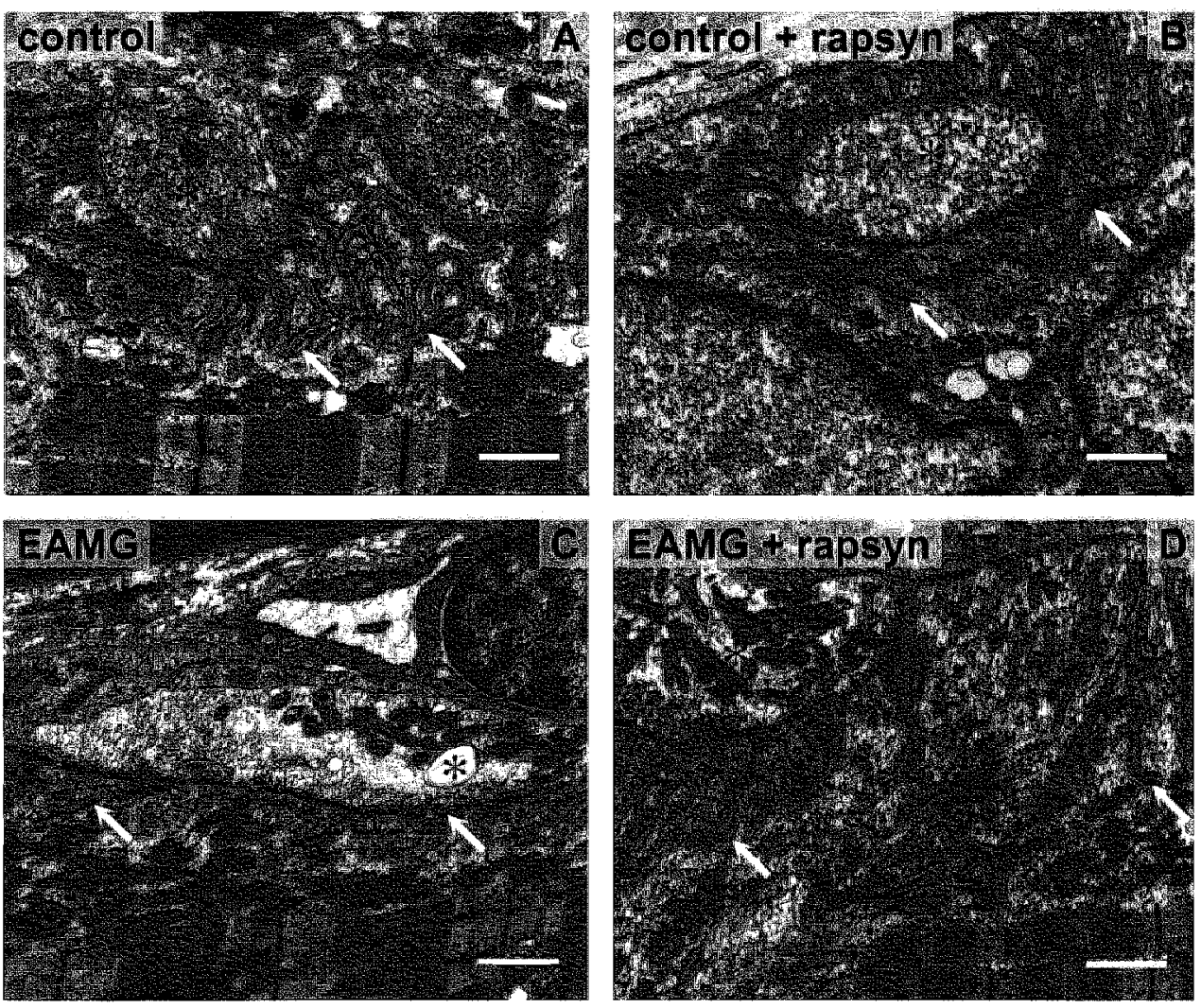

Figure 5. Electron microscopic examination of the postsynaptic folds of motor endplates. NMJ in an untreated (A) and rapsyn-treated (B) tibialis anterior muscle. Damaged postsynaptic membrane without folds in an untreated tibialis anterior muscle (C) of an EAMG animal. Normal postsynaptic membrane with folds in rapsyn-treated tibialis anterior (D) of an EAMG animal. Arrows indicate the postsynaptic membranes; stars indicate the nerve terminalls; scalle bars are $1 \mu \mathrm{m}$. 
rapsyn-treated EAMG muscles, only $8 / 49$ regions showed a complete destruction of the postsynaptic folding, 3 more regions were infiltrated by macrophages; therefore altogether 38/49 endplate regions (78\%) appeared normal. Moreover, the average length of the postsynaptic mentbrane in rapsyn-treated EAMG endplates was reduced only slightly compared to normal endplates (difference not significant, $p=0.09$ ), and the ratio of postsynaptic to presynaptic membrane length was normal $(p=0,85)$. An example of a well preserved endplate is shown in Figure 5D. There was a very significant difference between the postsynaptic membrane length of the rapsyn-treated EAMG endplates and the untreated EAMG endplates ( $p<0.0001$ ).

\section{Discussion}

Myasthenia gravis is caused by antibody-mediated loss of AChRs at the neuromuscular junction and most treatments are aimed at modulating the immune response. However, previous observations in rats suggested that intrinsic differences in expression of rapsyn, the $A C h R$ anchoring protein, may modify susceptibility to EAMG induced by immunisation with $A C h R$ or by passive transfer with antibody to $\mathrm{AChR}$. To prove that rapsyn alone was capable of modifying susceptibility, we transfected rapsyn unilaterally into the tibialis anterior muscles of EAMG rats. The rapsyn-treated muscles showed normal numbers and distribution of AChRs and lack of the morphological changes that typify the experimental and human disease, despite the presence of the membrane attack complex of complement. Thus these results imply that even in the presence of an active immune response against the AChRs, upregulation of rapsyn at the neuromuscular junction can substantially protect from antibody-induced AChR loss.

Electropermeabilisation of tibialis anterior muscles with pcDNA-rapsyn led to rapsyn overexpression in transfected muscle fibres, ranging from a few large extrasynaptic rapsyn aggregates to small rapsyn aggregates densely distributed throughout the muscle fibre cytoplasm. The amount of AChR in rapsyn-treated normal muscles was increased by $42 \%$ after two weeks, as measured by RLA of total muscle extracts. Similarly, a $50 \%$ increase in AChR was found at the cell surface when rapsyn was coexpressed in COS-7 cells (Marchand et al, 2002). The increased AChR concentration in rapsyn-treated muscles was likely due to the metabolic stabilizing effects of rapsyn (Gervasio and Phillips, 2005; Phillips et al., 1997; Wang et al., 1999) and is unlikely to be attributable to upregulation of $A C h R$ subunits because AChR gene expression is rapsyn independent (Gautam et al., 1995; Graus et al., 1993; Hoedemaekers et al., 1998). Electroporation of plasmid DNA alone does not lead to a change of $\mathrm{AChR}$ concentration in muscles, neither does it protect against $\mathrm{mAb} 35$ induced AChR loss (our unpublished results).

Rapsyn overexpression prevented AChR loss and muscle weakness in EAMG rats, which was confirmed by electrophysiological measurements which showed no decrement of the CMAP. Interestingly, this was not just the result of stabilisation of the AChRs themselves, but probably also related to lack of morphological damage since $78 \%$ of postsynaptic regions of rapsyn-treated muscles of EAMG rats were not damaged or infiltrated, as shown 
by EM studies, whereas the endplates of untreated EAMG muscles were all damaged, as expected. Engel and coworkers found that the postsynaptic membrane length in EM sections was reduced to $26 \%$ by day 5 after passive transfer of polyclonal rat anti $A C h R$ antibodies (Engel et al., 1979). Our ultrastructural data indicate that $\mathrm{mAb} 35$ reduces the postsynaptic membrane to $36 \%$, even as early as day 2 , and that rapsyn overexpression prevents this antibody-mediated destruction of postsynaptic folds.

$A C h R$ loss is also not seen in aged $B N$ rats subjected to passive transfer of EAMG (Graus et al., 1993). These animals, which have altered levels of several postsynaptic proteins, have a $25 \%$ increase in rapsyn:AChR ratio (Hoedemaekers et all, 1998). Our results suggest that AChR levels can be protected from antigenic modulation and complementmediated lysis by overexpressing rapsyn alone. Interestingly, we found much less infiltrating cells at endplates in rapsyn transfected muscles, which is another parallel to the age related resistance in EAMG. However, AChR loss in passive transfer EAMG is not primarily determined by infiltrating macrophages (Hoedemaekers et al, 1997a).

One possible explanation for rapsyn induced EAMG resistance is an increased rapsyn: $A C h R$ interaction. In cell culture studies, endogenous rapsyn and AChR are present in equimolar concentrations (Burden et al., 1983; LaRochelle and Froehner, 1986) but about $50 \%$ of rapsyn and AChR are not localised in complexes (Marangi et al., 2001). The 1:1 stoichiometry of rapsyn and AChR in cell extracts probably does not reflect the interaction of rapsyn and AChR focally at the endplate (Moransard et al., 2003; Tsui et al., 1990), which is more pronounced and is increased by agrin (Moransard et al., 2003). Rapsyn overexpression could stabilise the pool of unclustered AChR by increasing the level of AChR-rapsyn complexes. These could serve as a pool for cluster formation or maintenance, reducing the susceptibility to antigenic modulation. In rat muscles, we found an average increase of $20 \%$ in the rapsyn: $A C h R$ ratio; with some individual endplates reaching a $100 \%$ increase of rapsyn:AChR ratio, as measured by semiquantitative immunohistochemistry (Figure $2 \mathrm{D}$ ). Thus, in the intact muscle, since each of the five AChR subunits can bind rapsyn (Maimone and Merlie, 1993), the increased ratio of rapsyn molecules to AChRs might increase the linkage of the AChRs to the cytoskeleton and reduce its susceptibility to antigenic modulation. Interestingly, the increased rapsyn was also associated with resistance to complementmediated lysis, indicating that these mechanisms are interdependent in wiwo as previously suggested (Christadoss, 1988; Lennon and Lambert, 1981; Lennon et al., 1978).

Another possibility is that EAMG damaged endplates cannot recover from on-going antibody-mediated destruction because rapsyn is not available in sufficient amounts. This seems likely since in passive transfer EAMG the mRNA levels of the AChR subunits are upregulated, while rapsyn expression remains constant (Asher et al., 1993), presumably because rapsyn gene expression in vivo is controlled independently of $A C h R$ gene expression (de Kerchove D'Exaerde et al., 2002; Rodova et al., 2004). Therefore, a relative lack of rapsyn could restrict the replacement of other endplate proteins in EAMG; these might include those that link newly produced $A C h R$ to the cytoskeleton, perhaps rendering the AChR more susceptible to complement mediated lysis and antigenic modulation. 
The results presented here suggest that even a relatively small upregulation of rapsyn expression in MG patients might be therapeutically helpful. The electroporation of sielected muscles in humans is feasible, but probably too complicated using the current technical procedure. However, in the future improved vectors might become available for human gene-therapy. Alternatively, future studies might investigate the possibility to increase the expression of rapsyn by modulating synaptic transcription factors such as Kaiso (Rodova et al, 2004): Moreover, one could speculate that differences in endogenous rapsyn expression might be a factor in determining susceptibility of different muscles to MG within an individual, and polymorphic variants might be related to disease severity between individuals. It will be interesting to look for rapsyn expression quantitatively at the human neuromuscular junctions in different muscles, to determine what controls its level of expression, and to see whether polymorphisms in the promoter or coding reglons modify disease severity in $M G$.

\section{Acknowledgements}

We are very grateful to Dr. Christian Fuhrer (Brain Research Institute, University of Zürich, Switzerland) for the critical review of this manuscript, to Dr. J. T. Vilquin (Institut National de la Santé et de la Recherche Médicale U-582, Institut de Myologie, Groupe Hospitalier Pitie-Salpetriere, Paris, France) for the helpful discussions concerning electropermeabilization and to Marc van Zandvoort, Wim Engels (Department of Biophysics, Universiteit Maastricht, The Netherlands) and Christoph Schmitz (Department of Psychiatry and Neuropsychology, Universiteit Maastricht, The Netherlands) for their help with two photon confocal microscopy and image deconvolution. We would also like to thank $\mathrm{M}$. van de Waarenburg and H. P. J. Steinbusch for their excellent technical assistance. The BioRad TPLSM was obtained by grant no. 902-16-276 from the Medical Section of the Dutch Scientific Organization (NWO). This work was supported by grants from Prinses Beatrix Fonds and IAssociation Française contre les Myopathies and a Marie Curie Fellowship of the European Community program "Quality of لife and management of living resources".

\section{References}

Asher $\mathrm{O}$, Kues WA, Witzemann V. Tzartos SI, Fuchs S, Souroujon MC. Increased gene expression of acetylcholine receptor and myogenic factors in passively transferred experimental autoimmune myasthenia gravis. I Immunol 1993; $151: 6442-50$,

Banwell BL, Ohno K, Sieb JP, Engel AG. Novel truncating RAPSN mutations causing congerital myasthenic syndrome responsive to 3,4 -diaminopyridine. Neuromascul Disord $2004 ; 14: 202-7$.

Bloch $\mathbb{R}$, Froehner $S C$. The relationship of the postsynaptic $43 \mathrm{~K}$ protein to acetylcholine receptors in receptor clusters isolated from cultured rat myotubes. J Cell Biol 1987; 104:645-54.

Burder $S D_{2}$ DePalma RL, Gottesman GS. Crosslinking of proteins in acetylcholine receptor-rich membranes: association between the beta-subumit and the $43 \mathrm{kd}$ subsynaptic protein. Cell $1983 ; 35: 687-92$. 
Burke $G$, Cossins 7, Maxwell $S$, Owens $G$, Vincent $A$, Robb $S$, al Rapsyn mutations in hereditary myasthenia: distinct early-and late-onset phenotypesin Neurology $2003 ; 61: 826-8$.

Christadoss P. C5 gene influences the development of murine myasthenia grawis. J Immnol $1988,140,2589-92$.

De Baets $M_{0}$ Stassen $M H$. The role of antibodies in myasthenia grawis. I Neurol Sci $2002 ; 202,5-11$.

de Kerchove D'Exaerde A, Cartaud I, Ravel Chapus A, Seroz T, Pasteau $\mathrm{E}$ Angus LM, et al. Expression of mutant Ets protein at the neuromuscular synapse causes alterations in morphology and gene expression. EMBO Rep 2002; 3: 1075-81.

Dume $V$. Maselli RA. Identification of pathogenic mutations in the human rapsyn gene. J Hum Genet $2003 ; 4:$ $204-7$.

Engel AG, Sakakibara H, Sahashi K, Lindstrom JM, Lambert EH, Lermon VA. Passively transterred experimental atutoimmune myasthenia gravis. Sequential and quantitative study of the motor end-plate fine structure and ultrastructural localization of immune complexes ( $\mathrm{gGG}$ and $\mathrm{C3}$ ), and of the acetylcholine receptor. Neurology $1979 ; 29: 179-88$.

Engel AG, Tsujihata M, Lindstrom JM, Lennon $V A$. The motor end plate in myasthenia gravis and in experimental autoimmune myasthenia gravis. A quantitntive ultrastructural study. Ann N Y Acad Sci 1976; 274" 60 79.

Gautam M, Noakes PG, Mudd J, Nichol M, Chu GC, Sanes IR, et al. Failure of postsynaptic specialization to develop at neuromuscular junctions of rapsyn-deficient mice. Nature $1995 ; 377: 232-6$.

Gervasio $O L$, Phillips WD. Increased ratio of rapsyn to ACh receptor stabilizes postsynaptic receptors at the mouse neuromuscular synapse. I Physiol 2005; 562:673-85.

Glass DJ Bowen DC, Stit: TN, Radziejewski C, Bruno J, Ryan TE, el al. Agrin acts via a MusK receptor conaplex. Cell 1996; 85: 513-23.

Graus YM, Verschuuren JI, Spans F, Jennekens F, wan Breda Vriesman PI, De Baets MH. Age-related resistance to experimental autoimmune myasthenia gravis in rats. J Immunol 1993; 150: 4093-103.

Hoedemaekers A, Besserean IL, Graus Y, Guyon T, Changetux JP, Berrih-Alunin S, et al. Role of the target organ in determining susceptibility to experimental autoimmune myasthenia gravis. I Neuroimmunol 1998; 89 : $13]-41$.

Hoedemaekers A, Graus Y, Beijleveld L, van Breda Vriesman P, De Baets M. Macrophage inflitration at the neuromuscular junction does not contribute to AChR loss and age-related resistance to EAMG. I Neuroimmumol 1997a; 75: 147-55.

Hoedemaekers A, Graus Y, van Breda Vriesman P, de Baets M. Age-and sex-related resistance to chronic experimental autoimmune myasthenia gravis (EAMG) in Brown Norway rats. Clin Exp Immunol 1997b; 107 : $189-97$.

Hoedemaekers AC, Verschuuren IJ, Spaans D, Graus YF, Riemersma S, van Breda Vriesman PJ, ef al. Age-related susceptibility to experimental autoimmune myasthenia grawis: immunological and electrophysiological aspects. Muscle Nerve $1997 \mathrm{c} ; 20: 1091=1.01$.

Kimura J. Electrodiagnosis in diseases of nerve and muscle: principles and practice. Oxford: Oxford University Press, 2001.

LaRochelle WD, Froehner SC. Determination of the tissue distributions and relative concentrations of the postsynaptic 43-kDa protein and the acetylcholine receptor in Torpedo. Biol Chem 1986; 261:5270-4.

Lennon VA, Lambert EH. Monoclonal autoantibodies to acetylcholine receptors: evidence for a dominant idiotype and requirement of complement for pathogenicity. Ann $\mathrm{N} Y$ Acad Sci 1981;377:77-96. 
Lennon VA, Lindstion TM, Seybold ME. Experimental autoimmune myasthenia: A model of myvasthenia grawis in rats and guinea pigs. I Exp Med 1975; 141: 1365-75.

Lennon VA, Seybold ME, Lindstrom IM, Cochrane C, Ulevitch R. Role of complement in the pathogenesis of Experimental autoimmune myasthenia gravis. I Exp Med 1978; 147: 973-83.

Lindstrom IM, Engel AG, Seybold ME, Lennon VA, Lambert EH. Pathological mechanisms in experimental autoimnutie nyasthenila gravis. II. Passive transfer of experimental autoimmune myasthenia gravis in rats with antinacetylcholine recepotr antibodies. J Exp Med 1976a: 144: 739-53.

Lindstron M, Lennon VA, Seybold ME, Whittingham S. Experimental autoimmume myasthenia gravis and myasthenia gravis: biochemical and immunochemical aspects. Ann N Y Acad Sei 1976b; 274: 254-74,

Maimone MM, Merlie JP. Interaction of the $43 \mathrm{kd}$ postsynaptic protein with all subunits of the muscle nicotinic acetylcholine receptor. Neuron 1993; 11:53-66.

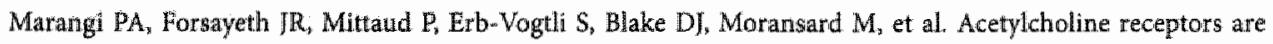
required for agrin-induced elustering of postsynaptic proteins. Embo f 2001; 20:7060-73.

Marchand S, Devillers-Thiery A, Pons $S_{*}$ Changeux IP, Cartaud J. Rapsyn escorts the nicotinic acetylcholine receptor along the exocytic pathway via association with lipid rafts. I Neurosci 2002; 22: 8891-901.

Mir LM, Bureau MF, Gehl I, Rangara $\mathrm{R}_{\Perp}$ Rouy $\mathrm{D}$, Caillaud IM, et al. High-efficiency gene transfer into skeletal muscle mediated by electric pulses. Proc Natl Acad Sci U S A 1999; 96: 4262-7.

Moransard M Borges LS, Willmann R, Marangi PA, Brenner HR, Ferns MI, et al. Agrin regulates rapsyn interaction with surface acetylcholine receptors, and this underlies cytoskeletal anchoring and elustering. I Biol Chem 2003; 278: 7350-9.

Muller IS, Abicht A, Burke $G$, Cossins J, Richard $P$, Baumeister $S K$, et al. The congenital myasthenic syndrome mutation RAPSN N88K derives from an ancient Indo-European founder. J Med Genet 2004; 41: e104.

Ohno $K_{n}$ Engel AG, Shen XM, Selcen D, Brengman J, Harper CM, et all. Rapsyn Mutations in Humans Cause Endplate Acetylcholine-Receptor Deficiency and Myasthenic Syndrome. Am J Hum Genet 2002; 70:4.

Ohno $\mathrm{K}$, Sadeh M, Blatt I, Brengman IM, Engel AG. E-box mutations in the RAPSN promoter region in eight cases with congenital myasthenic syndrome. Hum Mol Genet 2003; 12:739-48.

Phillips WD, Whideta $\mathrm{D}$, Han H, Noakes PG. Rapsyn and agrin slow the metabolic degradation of the acetylcholine receptor. Mol Cell Neurosci 1997; 10: 16-26.

Rodova M, Kelly KF, VanSaun M, Daniel IM, Werle MJ. Regulation of the rapsyn pronoter by kaiso and deltacatenin. Mol Cell Biol 2004; 24: 7188-96.

"Isui $\mathrm{HC}_{\mathrm{C}}$ Cohen IB, Fischbach GD. Variation in the ratio of acetylcholine receptors and the $\mathrm{Mr} 43,000$ receptorassociated protein in embryonic chick myotubes and nyoblasts. Dev Biol 1990; 140: 437 46.

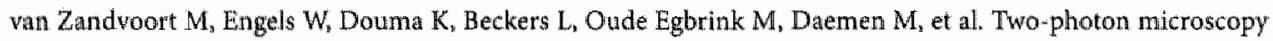
for imaging of the (atherosclerotic) wascular wall: a proof of concept study. I Vasc Res 2004; 41: 54-63.

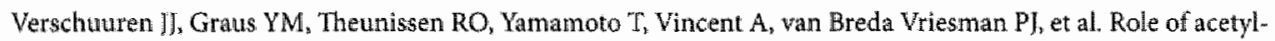
choline receptor antibody complexes in muscle in experimental autoimmune nnyasthenia gravis. J Neuroimimunoll 1992; 36: 117-25.

Verschuuren II, Spaans E, De Baets MH. Single-fiber electromyography in experimental autoimmune myasthenia gravis. Muscle Nerve $1990 ; 13: 485-92$.

Wang $\mathrm{ZZ}$, Mathias A, Gautam $\mathrm{M}_{\mathrm{v}}$ Hall ZW. Metabolic stabilization of muscle nicotinic acetylcholine receptor by rapsyn. I Neurosci 1999; 19: 1998-2007. 


\section{Chapter 3}

Overexpression of Rapsyn in Rat Muscle Increases Acetylcholine Receptor Levels in Chronic Experimental Autoimmune Myasthenia Gravis

Mario Losen, Pilar Martínez-Martínez, Hans, Duimel, Peter Frederik, Frank Spaans, Peter Molenaar, Angela Vincent and Marc H. De Baets 


\section{Abstract}

The primary autoantigen in myasthenia gravis, the acetylcholine receptor (AChR), is clusitered and anchored in the postsynaptic membrane of the neuromuscular junction by rapsyn. Previously, we have found that overexpression of rapsyn by CDNA transfection confers resistance to subsequently induced experimental autoimmune myasthenia gravis (EAMG) in rat muscle. As a next step we investigated here the effect of rapsyn overexpression once the EAMG-induced damage is underway. It was found, despite of the presence of high titers of anti-AChR anti-bodies, that the rapsyn overexpression resulted in an increase of total muscle membrane AChR levels. This increased AChR was localized both at endplates and in extrasynaptic membrane aggregates, and found to be bound to rat anti-AChR anti-bodies. Immunohistochemical analysis showed that endplates in transfected EAMG fibers with a high level of rapsyn recovered from $A C h R$ loss and were comparable to endplates from healthy animals, but most if not all of the increase of $A C h R s$ took place in the perisynaptic region. At the ultrastructural level, endplates in rapsyn-treated muscles frequently showed increased damage of the postsynaptic membrane. The results suggest a molecular mechanism of the disease where rapsyn overexpression stabilizes AChRs in intact or mildly damaged endplates, but, on the other hand, enhances membrane damage when the antibody attack is in progress.

\section{Introduction}

The molecular organization of the neuromuscular junction (NMJ) is designed for optimal reliability of the process of neuromuscular transmission (Sanes and Lichtman, 1999). In myasthenia gravis (MG), the muscular nicotinic acetylcholine receptor ( $A C h R$ ) is the main auto-antigen and consequently the postsynaptic membrane of the NMT is the target for antibody-induced damage. AChR antibodies are found in $85 \%$ of MG patients, while some AChR antibody seronegative patients have, instead, auto-antibodies against the musclespecific kinase (MuSK) (Hoch et al., 2001).

The AChRs are clustered in high density at the motor endplate. However, in MG, antibodies cause loss of functional AChRs at the motor endplate, either by cross-linking the receptors, leading to increased turnover of the $A C h R$, activation of complement, leading to focal loss of the postsynaptic membrane folding, or by blocking the AChR's ion channel (e.g. see review De Baets and Stassen, 2002). Loss of functional AChRs compromises the reliability of neuromuscular signal transmission, resulting in skeletal muscle weakness.

A pivotal protein necessary for the stability of the AChR in the membrane is rapsyn, a $43 \mathrm{kD}$ membrane protein, which is essential for the formation of the postsynaptic apparatus (Gautam et al.. 1995). The AChR and rapsyn are co-transported to the postsynaptic membrane via post-Golgi vesicular transporters (Marchand et al., 2000). The clustering of postsynaptic proteins is initiated by agrin, a neuronal protein which is acting via a receptor complex including MuSK (Glass et al., 1996). Agrin triggers phosphorylation of both MuSK and $A C h \mathbb{R}$ resulting in the clustering and anchoring of preassembled AChR-rapsyn 
complexes to the cytoskeleton (Moransard et al, 2003). Mice deficient in rapsyn suffocate at birth because the postsynaptic specialization of the NMJ fails to develop (Gautam et al., 1995). Besides being essential for clustering, rapsyn metabolically stablizes the $A C h R$; cotransfection of rapsyn and AChR expression plasmids increases the half-life of AChR in cell lines (Phillips et al, 1997; Wang et al., 1999), and rapsyn also reduces antigenic modulation of AChRs in transfected fibroblasts when incubated with the anti-AChR monoclonal antibody (mAb) 35 (Phillips et al., 1997).

Experimental autoimmune MG (EAMG) is an animal model which closely resembles clinical MG (Lennon et al., 1975). EAMG can be induced by passive transfer of MG patient sera or anti-AChR $m A b s$, or can be induced by immunization with $t-A C h R$ derived from Torpedo electric organ (chronic EAMG), because some antibodies against $\mathrm{AAChR}$ are cross-reacting with the rat $\mathrm{AChR}$. Antigenic modulation and complement-mediated focal damage of the postsynaptic membrane structure are the pathogenic mechanisms of EAMG, as in MG itself. The symptoms include weight loss due to impaired swallowing ability, hunched posture, drooping of the head and a weak grip of the forelimbs. Age and sex dependent resistance to the induction of passive and chronic EAMG has been observed in Lewis and BN rats (Graus et al., 1993; Hoedemaekers et al., 1997b; Hoede. maekers et al., 1997c). This resistance is not due to differences of the immune response or compensatory mechanisms such as increased expression of AChRs or complement modulatory proteins (Hoedemaekers et al., 1997a; Hoedemaekers et al., 1997b; Hoedemaekers et al., 1997c). In fact, the postsynaptic membrane is intrinsically resistant to antibodymediated degradation in aged animals (Hoedemaekers et al., 1998). Interestingly, in aged rats, rapsym levels are increased relative to those of the AChRs, suggesting that rapsyn can make the AChRs in the membrane more resistant to antibody-induced degradation (Hoedemaekers et al., 1998). Recently, we found that increased expression of rapsyn in muscle fibers using in wivo gene transfection induced by $\mathrm{cDNA}$ electroporation protects against subsequent induction of passive transfer EAMG (Losen et al., 2005); in rapsyntransfected muscles the AChR loss and the ultrastructural damage of the endplates are prevented. Furthermore, the absence of decrement of the compound muscle action potential after repetitive nerve stimulation indicates that the neuromuscular transmission is preserved after overexpression of rapsyn.

In the present study we tested the effect of rapsyn overexpression in rat muscle at a time when the level of AChRs is down during ongoing chronic EAMG, in order to see whether overexpression can reverse the situation of $A C h R$ depletion. In this way we aimed to better understand the role of rapsyn in the pathogenicity of antibodies in $\mathrm{MG}$.

\section{Methods}

Animals. 6-week-old female Lewis rats were obtained from the Department of Experimental Animal Services, University of Maastricht, The Netherlands, with permission of the Committee on Animal Welfare, according to Dutch governmental rules. For immu- 
nization, in wivo electroporation and electromyography measurements, the animals were anesthetized with $3 \%$ isofurane in air; supplied over a cylindrical cap held over the head. The animals were euthanized by $\mathrm{CO}_{2}$ /air inhalation and subsequent cervical dislocation.

Induction of chronic EAMG. The animals were immunized in the base of the tail with 20 ug of tAChR in $0.1 \mathrm{ml}$ phosphate-buffered saline (PBS) emulsified in an equal amount of complete Freund adjuvant (Difco Laboratories, Detroit, USA) (Lennon et al., 1975). Blood samples were taken from the tip of the tail wreekly, and additionally the weight of the animal was recorded. After 5 weeks the rats were clinically scored, anesthetized for electropermeabilization and euthanized 2 weeks later.

Ellectropermeabilization. The expression plasmid pcDNA1.1-rapsyn (Losen et al., 2005) was prepared for electropermeabilization with the Qiagen Maxiprep, according to the manufacturer's manual, and finally dissolved in $0.9 \% \mathrm{NaCl}$ at a concentration of $2 \mu \mathrm{g}$ DNA/ $\mu$ l. $75 \mu \mathrm{l}(2 \mu \mathrm{g} / \mu \mathrm{l})$ pcDNA-rapsyn was injected in aliquots at $7-8$ sites equally spread over the muscle and electropermeabilized in the tibialis anterior with the same parameters as previously described (Losen et al., 2005) using the Electro Square Porator ECM 830 (BTX, San Diego, USA) (Mir et al., 1999).

Clinical scoring. The severity of clinical signs of disease in EAMG was scored by measuring muscular weakness. The animals' muscle strength was assessed by their ability to grasp and lift repeatedly a $300 \mathrm{~g}$ rack from the table, while suspended manually by the base of the tail for 30 seconds (Hoedemaekers et al., 1997b; Hoedemaekers et al., 1997c; Verschuuren et al, 1990). Clinical scoring was based on the presence of tremor, hunched posture, muscle strength and signs of fatigue. Disease severity was expressed as: 0 , no obvious abnormalities; + , no abnormalities before testing, but reduced strength at the end; ++ , clinical signs present before testing, i.e. tremor, head down, hunched posture, weak grip; +++ , severe clinical signs present before testing, no grip, moribund (Lennon et al., 1975).

Electromyography (EMG). Decrement of compound muscle action potential (CMAP) was measured in the tibialis anterior muscles of 5 EAMG rats as previously described (Losen et al., 2005). To detect a decrementing response, series of 8 supramaximal stimuli were given at $3 \mathrm{~Hz}$. Stimulus duration was $0.2 \mathrm{~ms}$. This test was repeated 10 minutes after injection of 3 to $6 \mu \mathrm{g}$ of curare (Seybold et al., 1976). The test was considered positive for decrement when both the amplitude and the area of the negative peak of the CMAP showed a decrease of at least $10 \%$ (Kimura, 2001). To demonstrate reproducibility, at least 3 recordings were made of all investigated muscles. During the measurements, skin temperature was kept between $35^{\circ} \mathrm{C}$ and $37^{\circ} \mathrm{C}$ by means of a heating pad. All EMG studies were performed by the same investigator (F.S.).

Immunohistochemical staining. Isolated tibialis anterior muscles of 3 control and 6 EAMG animals were frozen in melting isopentane. Cryosections of 10 um were dried, fixed in acetone at $4^{\circ} \mathrm{C}$ for $10 \mathrm{~min}$, dried and blocked for 20 min with PBSA (phosphate-buffered saline with $2 \%$ bovine serum albumin). Sections were incubated with the following antibodies: mouse anti-rapsyn mAb 1234 (1/500 in PBSA; Affinity Bioreagents, Golden, USA) (Bloch and Froehner, 1987); mAb $2 \mathrm{~A} 1$ against rat C5b-9 (membrane attack complex, 1/100 
in PBSA; kindly provided by W.G. Couser, University of Washington, USA), rabbit anti-vesicular acetylcholine transporter (VAChT; 1/500 in PBSA Phoenix Pharmaceuticals, Belmont, USA); mouse anti-utrophin mAb MANCHO 7 (1/100 in PBSA; kindly provided by Prof. G.E. Morris, North East Wales Institute, Wrexham, UK) (Nguyen et al., 1991) and Alexa 594-conjugated a-bungarotoxin (Alexa 594-a-BT; dilluted 1/300 in PBSA: Molecular Probes, Leiden, The Netherlands) overnight at $4^{\circ} \mathrm{C}$ and finally washed with PBS with $0.05 \%$ Triton-X100. Subsequently the sections were incubated for $1.5 \mathrm{~h}$ at room temperature with the corresponding secondary antibodies: biotinylated donkey anti-mouse Ig (1/400; minimal cross-reaction with rat IgG; Jackson Immunoresearch, West Groove, USA) or Alexa 350-conjugated goat anti-rabbit (1/100; Molecular Probes). After washing as described above, the biotinylated antibodies were stained for $1.5 \mathrm{~h}$ at room temperature with Alexa 488-streptavidin (1/2000; Molecular Probes, Leiden, The Netherlands). Coverslips were mounted with $0.2 \mathrm{M}$ Tris $\mathrm{pH}=8$ with $80 \%$ (v/v) glycerol.

Quantitative immunofluorescence analysis, Pictures of muscle sections were taken using a Provis AX70 fluorescent microscope (Olympus, Hamburg, Germany) with a digital camera (U-CMAD-2, Olympus) and the AnalySIS software (Soft Imaging Systems, Münster, Germany). Sections triple stained for VAChT, rapsyn or utrophin, and AChR were photographed using filters for Alexa 350, FITC and Alexa 594 fluorescence. A five fold reduced concentration of primary antibodies and bungarotoxin did not result in a weaker staining, therefore they did not limit staining intensity. Pictures were analyzed using the Imagel software (version $1.33 \mathrm{n}$; http://rsb.info.nih.gov/ij). Endplate areas were identified by the presynaptic staining of VAChT and the mean intensity of VAChT, rapsyn or utrophin and AChR staining was measured in the corresponding area. The ratios of AChR:VAChT, rapsyn:VAChT and utrophin:VAChT were calculated for more than 150 endplates per muscle as a relative measure for the postsynaptic rapsyn, utrophin and AChR concentration. All sections were stained and processed in parallel to awoid inter-assay variations.

Two-photon laser scanning microscopy. High resolution pictures of endplates were taken using a two-photon laser scanning microscope setup as previously described (van Zandwoort et al., 2004). A 40x oil immersion objective with a numerical aperture of 1.0 was used. Further magnification was achieved by optical zoom ( $4 \mathrm{x}$ ) of the scan head. Picture stacks with a axial resolution of $0.15 \mu \mathrm{m}$ and a laterally resolution of $0.13 \mu \mathrm{m}$ were taken using photomultipliers accepting wavelengths of $470-500 \mathrm{~nm}, 520-560 \mathrm{~nm}$ and over $560 \mathrm{~nm}$. To remove noise each image was filtered applying the Kalman filtering procedure on two subsequent images. For projection of the images the ImageJ software was used.

Muscle membrane extracts. Tibialis anterior muscles were minced and homogenized with an Ultra-Turrax ( 3 times for $30 \mathrm{~s}$ at $4^{\circ} \mathrm{C}$ ) in $10 \mathrm{ml}$ Buffer A (PBS, $10 \mathrm{mM}$ EDTA, $10 \mathrm{mM} \mathrm{NaN3}, 10 \mathrm{mM}$ iodoacetamide, $1 \mathrm{mM}$ PMSF). Homogenate was centrifuged $(22100 \mathrm{~g}, 30 \mathrm{~min}$ ) and the resulting pellet was resuspended in $2.5 \mathrm{ml}$ Buffer $\mathrm{B}$ (Buffer A with additional $0.5 \%$ Triton $\mathrm{X}-100)$. Extraction was performed for $1 \mathrm{~h}$ at $4^{\circ} \mathrm{C}$ on a reciprocal shaker followed by centrifugation $(22100 \mathrm{~g}, 30 \mathrm{~min})$. The AChR was measured immediately by radioimmunoassay. 
Measurement of total muscle AChR by radioimmunoassay, The AChR concentrations of isolated tibialis anterior muscles from 5 control and 10 EAMG rats were measured according to the principle described by Lindstrom and colleagues (Lindstrom et al., 1976) with the following modifications. Triplicate aliquots of $200 \mu$ were taken from the muscle membrane extracts and incubated with an excess of [" ${ }^{125} \mathrm{I}$-Tyr54] a-bungarotoxin $\left.{ }^{125} \mathrm{I}-\mathrm{a}-\mathrm{BT}\right)$ and an excess of rat anti-rat-AChR: $20 \mu \mathrm{l}$ of Buffer A with 25 fmol ${ }^{125} \mathrm{I}-\mathrm{a}-\mathrm{BT}$ (74 TBq/mmol, GE healthcare, Rosendaal, The Netherlands) and 3 pmol of polyclonal rat

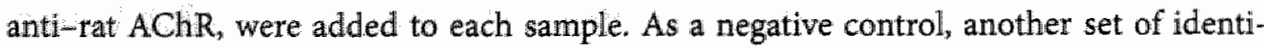
cally processed triplicate aliquots were supplemented with an excess of acetylcholine and neostigmine bromide $(10 \mu \mathrm{l}$ of Buffer $\mathrm{A}$ containing $1 \mathrm{mM}$ acetylcholine and $1 \mathrm{mM}$ neostigmine bromide per sample). After overnight incubation at $4^{\circ} \mathrm{C}$, the immune complexes were precipitated with goat anti-rat $\operatorname{Ig}(100 \mu \mathrm{l}$ serum) for $4 \mathrm{~h}$ and centrifuged at $15000 \mathrm{~g}$ for 5 minutes. The pellets were washed 3 times in PBS with $0.5 \%$ Triton $\mathrm{X}-100$ and measured in a $\gamma$-counter (Compugamma, Pharmacia LKB, Woerden). The AChR concentration per gram of fresh muscle weight was calculated in fmol/g \pm standard deviation and differences between concentrations are presented in percentage \pm standard error of the difference of the means. Differences between samples were analyzed using a two-sided t-test (paired differences were used for comparison of rapsyn-treated versus control in the same experimental group).

Measurement of AChR antibody complexes by radioimmunoassay The proportion of AChR bound to antibodies was measured as described before (Lindstrom et al., 1976; Verschuuren et al., 1992) with the following modifications. Triplicate aliquots of $200 \mu \mathrm{l}$ were taken from the muscle membrane extracts and each aliquot was incubated with an excess of ${ }^{125} \mathrm{I}-\mathfrak{a}-$ $B T$ and $1 \mu l$ normal rat serum. Additionally, in the extract of control muscles a limited amount of rat anti-rat-AChR antibody equivalent to $64 \mathrm{fmol} \mathrm{a}-\mathrm{BT}$ binding sites was added. A second set of triplicate aliquots was incubated without added anti-AChR antibodies and served as control. To the extract of EAMG muscles no rat anti-rat-AChR antibody was added. After overnight incubation at $4^{\circ} \mathrm{C}$, the samples processed using the aforementioned RIA protocol for total muscle AChR.

Measurement of serum anti-rat $A C h R$ antibody titers. Serum was obtained before immunization and afterwards weekly up to the euthanasia. Anti-rat $A C h R$ serum antibodies were measured using crude extract of denervated rat muscle $A C h R: 100 \mu$ of rat AChR extract (approximately $5 \mathrm{nmol} / \mathrm{L}$ ) were labeled with an excess of ${ }^{125} \mathrm{I}-\mathrm{a}-\mathrm{BT}$ ( $5.55 \mathrm{TBq} / \mathrm{mmol}$, GE healthcare) and incubated with $5 \mu \mathrm{l}$ rat serum at $4^{\circ} \mathrm{C}$ overnight. The resulting complexes were precipitated by addition of $100 \mu \mathrm{l}$ goat anti-rat Ig, $4 \mathrm{~h}$ incubation and centrifugation at $15000 \mathrm{~g}$ for $5 \mathrm{~min}$. The pellets were washed 3 times in PBS with $0.5 \%$ Triton $X-100$ and measured in a $\gamma$-counter. Titers were corrected for the background of normal rat serum. The antibody titer was expressed as moles of $a-B T$ binding sites per liter.

Electron microscopy. 3 EAMG rats and 3 control rats with unilateral-rapsyn treatment in the tibialis anterior were anesthetised with $60 \mathrm{mg} / \mathrm{kg}$ sodium pentobarbital and transcardially perfused with Tyrode solution $(0.1 \mathrm{M})$ followed by fixation buffer ( $2.5 \%$ glutaraldehyde in $0.1 \mathrm{M}$ 
phosphate buffer $\mathrm{pH}=7.4$ ). The tibialis anterior muscles were removed, postfixed for $2 \mathrm{hrs}$ and sectioned on a vibratome 1000 at $1 \mathrm{~mm}$. Sections were postfixed with $1 \%$ osmium tetroxide in 0.1 phosphate buffer, $\mathrm{pH} 7.4$, dehydrated through a graded ethanol series and embedded in epoxy resin (Glycid ether 100, Serva, Heidelberg, Germany). Endplates were located in toluidine bluestained semi-thin sections from the central region of each muscle. Ultra-thin sections from selected areas were contrasted with uranyl acetate and lead citrate and viewed with Philips CM 100 electron microscope. At least five endplate regions were photographed from each muscle: Pictures were scanned for morphometric analysis using the Imagel software. Analysed parameters included the size of nerve boutons and the length of the presynaptic and postsynaptic membrane (Engel et al., 1976a; Engel et al., 1976b).

\section{Results}

\section{Rapsyn overexpression increases the total muscle AChR concentration}

The effects of rapsyn transfection were studied in healthy animals and in the chronic EAMG model. To this end, 10 rats were immunized with $\triangle A C h R$, while 5 non-immunized rats served as controls. All tAChR immunized rats developed high titers of anti-rat AChR antibodies 5 weeks later (Figure 1, open symbols). At this time point, the left tibialis anterior muscles of all animals were transfected with pcDNA-rapsyn by electropermeabilization ("rapsyn-treated" muscles). The right tibialis anterior muscles were electropermeabilized

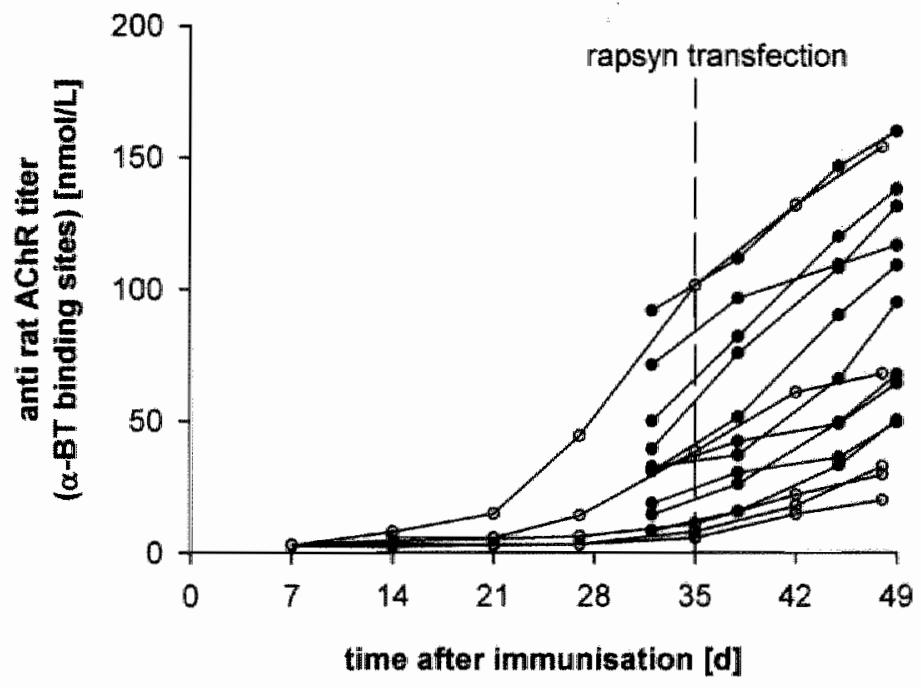

Figure 1. Time course of anti-rat AChR antibody titers in female Lewis rats immunized with 20 ug of Torpedo AChR in complete Freund's adjuvant at the age of 6 weeks (day 0 ). The tiblalis anterior muscles were electroporated 5 weeks later and analyzed 7 weeks post immumization. Titers with closed symbols belong to amimals used for the measurement of total AChR and AChR-antibody complexes in Figure 2 and 3 . Titers with open symbols correspond to animals immunized for immunofluorescence studies shown in Figures $4-9$. 
after injection of an equall wolume of saline ("untreated" muscles). Two weeks after transfection the animals were euthanized and the concentration of total $A C h R$ (synaptic and extrasynaptic AChR) was subsequently measured by radioimmunoassay in muscle membrane extracts from individual tibialis anterior muscles. The results are summarized in Figure $2 \mathrm{~A}$ and the correlation between $A C h R$ concentration and antibody titer is shown in Figure $2 \mathrm{~B}$.

Rapsyn-treated muscles of control animals contained significantly more $A C h R$ than untreated muscles (an increase of $36 \mathrm{fmol} / \mathrm{g}$, corresponding to $43 \% ; \mathrm{n}=5 ; \mathrm{p}=0.002$, paired $\mathrm{t}$-test)
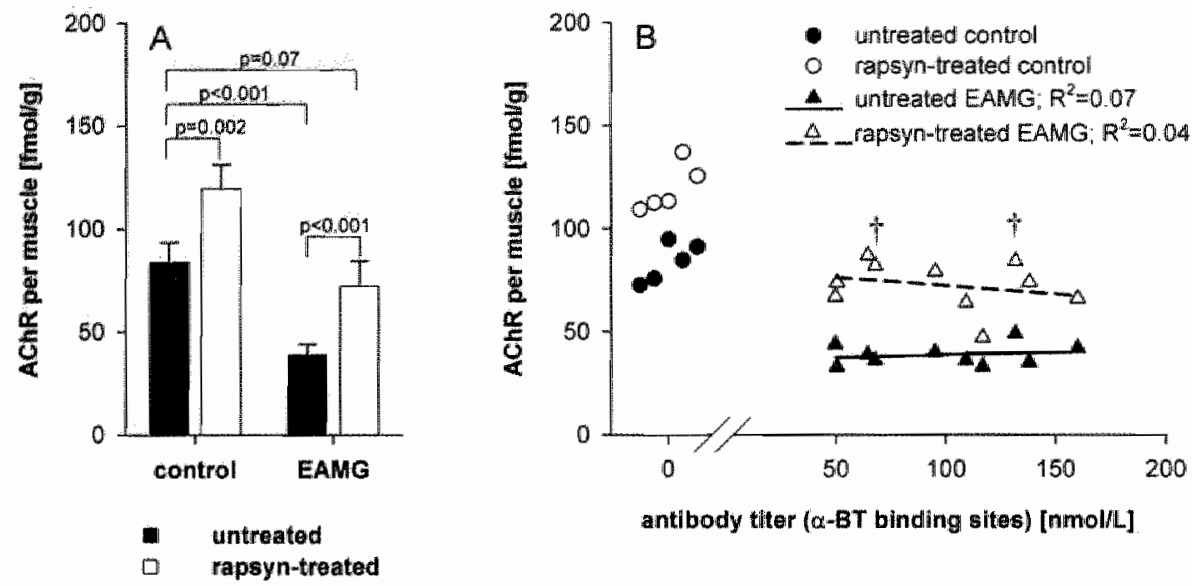

Figure 2. Effect of rapsyn overexpression on total membrane AChR concentration in control rats and in chronic EAMG rats. The AChR concentration of untreated and rapsyn-treated tibialis anterior muscles was measured in 5 control and 10 chronic EAMG rats by RIA using ${ }^{125}$ / Flabelled a-bungarotoxin. Average AChR concentration in the tiblalis anterior muscles in untreated muscles (black bars) and rapsyn-treated muscles (white bars) (A). The effect of anti-rat AChR antibody titer on AChR levels (B). Closed symbols indicate the total AChR of untreated muscles; open symbols correspond to total AChR of rapsyn-treated muscles. All rapsyn-treated muscles had an increased amount of total AChR compared with the untreated muscle of the same animal. Data points below the dagger belong to animals with severe weakness (score of +++ .

(Figure 2A, control). Electroporation of the empty vector did not change the AChR concentration compared to saline treated muscles (data not shown).

Untreated EAMG muscles showed a significant reduction of AChR compared to untreated control muscles $(-46 \% ; \mathrm{p}<0.001$, unpaired t-test). However, the contralateral rapsyn-treated EAMG muscles had only a small, but not significant reduction of AChR compared to untreated control muscles $(-14 \% ; \mathrm{p}=0.07$, unpaired t-test). Indeed, the $A C h R$ concentration in rapsyn-treated EAMG muscles was increased by $87 \%$ compared to the contralateral untreated EAMG muscles (an increase of $34 \mathrm{fmol} / \mathrm{g} ; \mathrm{p}<0.001$, paired $\mathrm{t}$-test; Figure 2A, EAMG).

Interestingly, there was no correlation between the antibody titer and the AChR loss of EAMG animals, both in the untreated and the rapsyn-treated EAMG muscles (Figure $2 \mathrm{~B}$ ). In this 
experiment, two animals developed clinical symptoms (grade ++ ; indicated with + in Figure $2 \mathrm{~B}$ ), but neither this was associated with high antibody titers nor increased AChR loss.

\section{Rapsyn overexpression increases antibody binding to AChR-rapsyn complexes}

As a measurement of the size of AChRs-rapsyn-clusters, the muscle membrane extracts of control animals were used for an immunoprecipitation experiment using a limited amount of rat anti-rat $A C h R$ antibodies (see Figure $3 A$ ). The addition of a limited amount of anti-AChR antibodies (corresponding to 64 fmol a-BT-bindung sites) precipitated approximately $23 \%$ of the total $\mathrm{AChR}$ in untreated control muscles, and $20 \%$ in the rapsyn-treated control muscles (Figure $2 \mathrm{~A}$, control + anti-AChR antibodies). In the absence of anti-AChR antibodies no $A C h R$ was precipitated in extracts from control animals, as expected (Figure $3 \mathrm{~B}$, control). This result indicates that rapsyn transfection did not lead to larger AChR clusters that could resist the extraction procedure.

A similar immunoprecipitation experiment was performed with muscle membrane extracts from chronic EAMG rats. In untreated EAMG muscles, $26 \%$ of AChR could be precipitated without the addition of anti-AChR antibodies, because circulating antibodies bound to the AChR already in vivo (Figure $3 \mathrm{~B}, \mathrm{EAMG}$ ). In the extracts of the contralateral rapsyn-treated EAMG

A
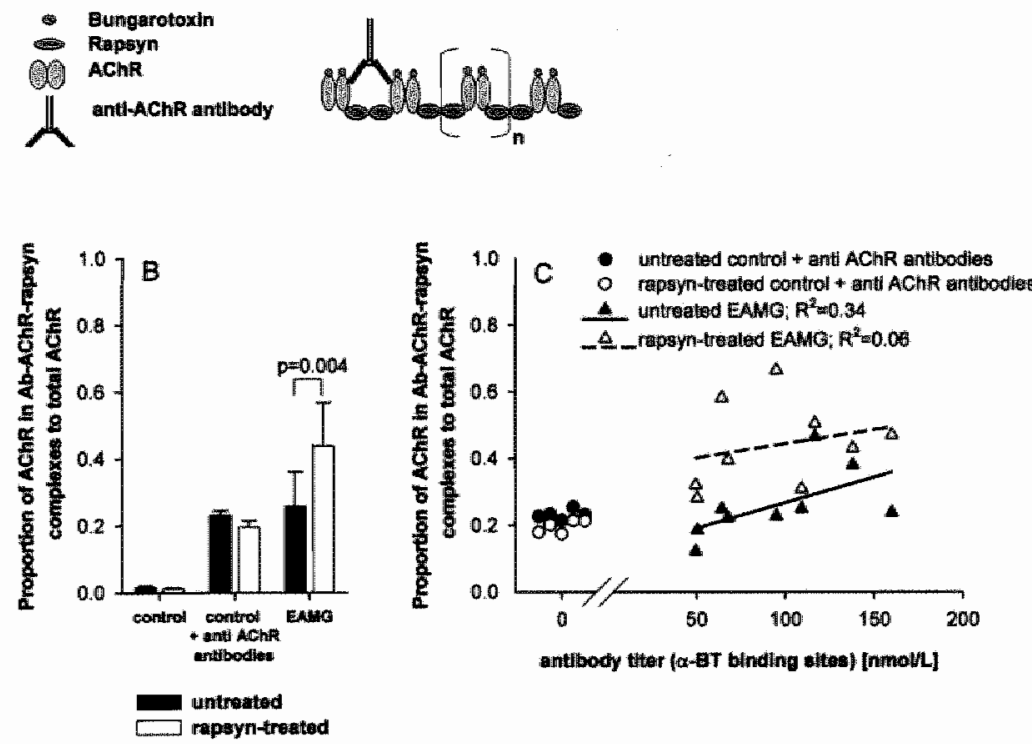

Figure 3. Measurement of antibody-AChR-rapsyn complexes by RIA using ${ }^{125}$ |-labelled a-bungarotoxim. The potential increase of $A C h R$ clustering by rapsyn was tested by adding a limited amount of anti-rat $A C h R$ to membrane extracts of untreated and rapsyn-treated controll muscles. Schematic representation of AChR complexes, showing AChR aggregation by rapsyn and AChR cross-linking by antibodles (A). Rapsyn transfection did not lead to increased size of rapsyn-AChR aggregates in membrane extracts of control muscles, but to increased binding of AChR to antibodies in EAMG animals (B). The proportion of AChR in AChR-antibody complexes is correlated to the antibody titer in EAMG animals (C). 
muscles this proportion was increased significantly to $44 \%(\mathrm{p}=0.004$, paired t-test). It is unlikely that this effect is caused by increased cross-linking of AChRs by rapsyn, because this was not observed in the rapsyn-treated control muscles. Therefore, the results suggest that a high proportion of AChR of rapsyn-treated EAMG muscles was bound to antibodies already in wiwo and that this AChRs resisted degradation. Because cytoplasmic AChR is not accessible for autoantibodies, this result indicates that rapsyn directed the $A C h R$ to the cell-membrane.

In untreated $E A M G$-muscles, there was a weak positive correlation $\left(R^{2}=0.34 ; p=0.045\right)$ between the antibody titer and the proportion of antibody-bound $\mathrm{AChR}$ (Figure $3 \mathrm{C}$ ), as also previously reported (Verschuuren et al., 1992). In rapsyn-treated EAMG muscles, there was no significant correlation $\left(\mathbb{R}^{2}=0.06 ; \mathrm{p}=0.24\right)$. However, in each animal, the proportion of $A C h R$ precipitated by antibodies was higher in the rapsyn-treated muscle than in the untreated muscle, even at high antibody concentrations.

\section{Rapsyn overexpression increases the amount of AChR and rapsyn at endplates and extrasyn- aptic AChR aggregates}

To examine further the changes at the neuromuscular junction we performed immunofluorescence studies in five EAMG and three control rats, immunized and electroporated. The immunized rats developed similar antibody titers (Figure $\mathbb{1}$, closed symbols) as in the previous experiment. The animals showed mild weakness after testing (grade + ) with the exception of the animal with the highest antibody titer, which developed severe clinical symptoms (grade $++)$. Cryosections of the isolated tibialis anterior muscles were triple-stained for rapsyn, AChR and VAChT.

In untreated muscles of control rats, $A C h R$, rapsyn and VAChT co-localized the newromuscular junctions (Figure $4 \mathrm{~A}$ ). Additionally, many muscle fibers from rapsyn-treated muscles of control rats had a large number of intensively stained extrasynaptic rapsyn aggregates of different sizes, localized in the cell-membrane (Figure $4 \mathrm{~B}$ ) and in intracellular aggregates, corresponding to the Golgi-vesicles (Gervasio and Phillips, 2005). Extrasynaptic rapsyn aggregates rarely co-localized with $\mathrm{AChR}$ in the cell membrane of transfected muscle fibers of control rats (not shown).

In chronic EAMG rats, AChR and rapsyn staning of endplates of the untreated muscle was weak, relative to the presynaptic VAChT staining (Figure $4 \mathrm{C}$ ). In the rapsyn-treated contralateral muscles, the endplates in fibers with intense staining of rapsyn also stained intensely for AChR (Figure 4D, endplate indicated with arrowhead). These were simillar to endplates of control muscles, but only represented approximately $1 \%$ of all endplates of the muscle. Endplates in fibers with a low amount of extrasynaptic rapsyn allso stained weakly for AChR (Figure 4D, endplate indicated by arrow). A large number of extrasynaptic membrane rapsyn-AChR aggregates were found in rapsyn-treated muscles from EAMG rats (Figure 4E), that were absent in the contralateral untreated muscles (4C). In all muscles of EAMG rats (untreated and ra* psyn-treated) intracellular AChR-rapsyn aggregates were frequently observed in synaptic areas (Figure 4F). 
$\mathrm{AChR}$
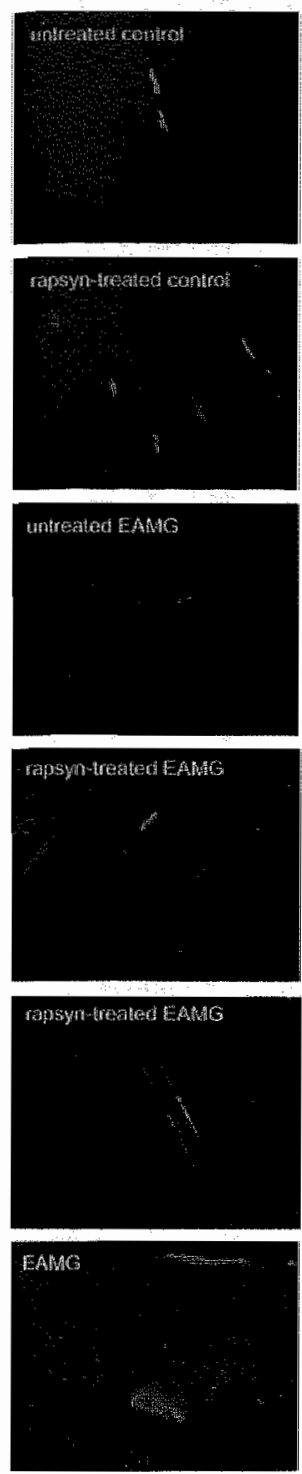

rapsyn
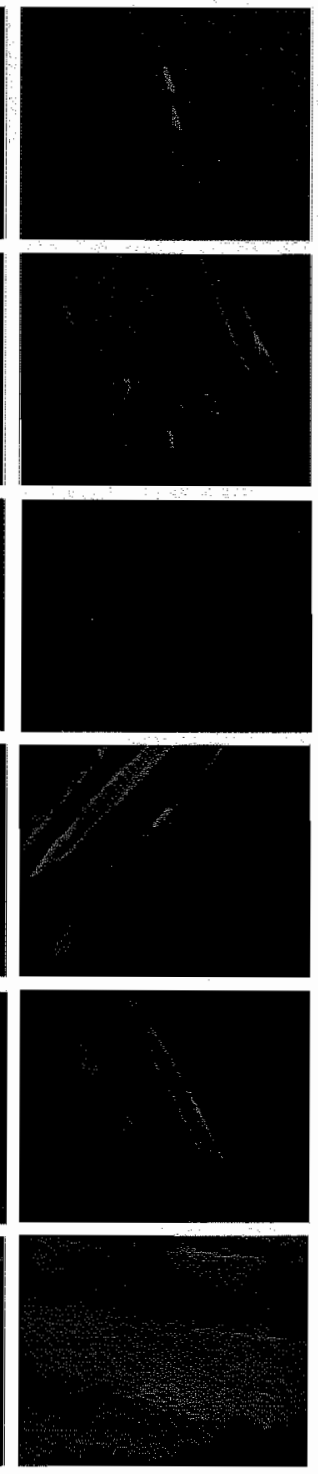

VAChT
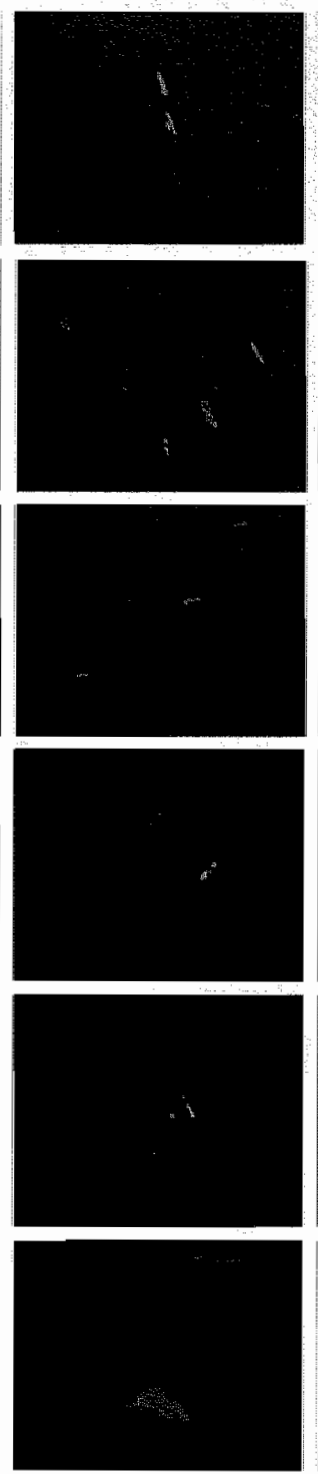

merge
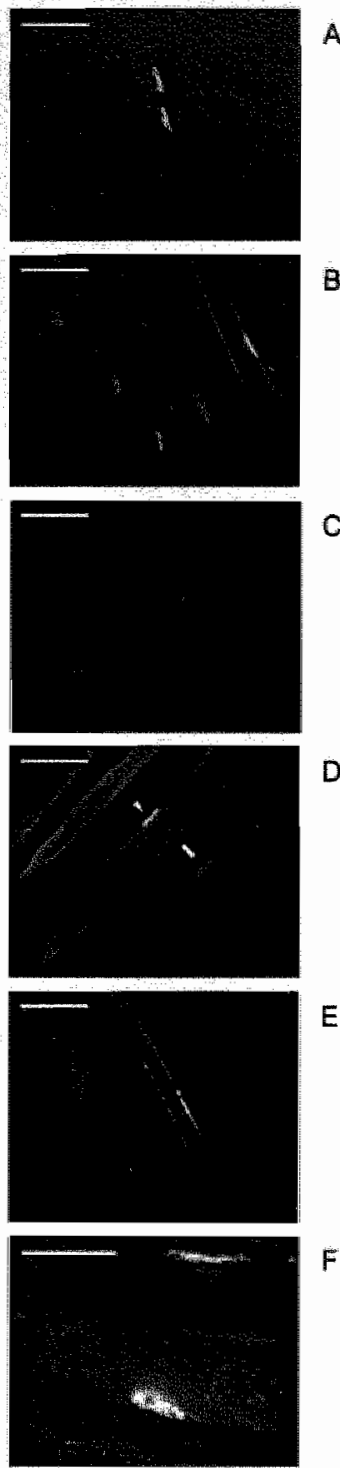

Figure 4. Cryosections of tibialis anterior muscles were triple-stained with Alexa 594-conjugated a-bungarotoxin (red), mouse anti-rapsyn mAb1234 (green) and rabbit anti-VAChT (blue); merge on the right. In an untreated tibialis anterior muscle of a control rat $A C h R$, rapsyn and VAChT are co-localized at the endplates (A). In the contralateral rapsyn-treated muscle of the same animal, rapsyn is localized extrasynaptically in the cell membrane of a transfected fiber. In this fiber, also the endplate contains an increased amount of rapsyn (B). In untreated chronic EAMG muscles, endplates showed reduced staining of AChR and rapsyn but not VAChT (C). Endplates with high amount of extrasynaptic rapsyn aggregates in rapsyn-treated EAMG muscle:5 had a staining intensity of $A C h R$ and rapsyn that was similar to endplates of untreated control muscles $(D$, arrowhead). In other fibers without such aggregates endplates had a reduced staining intensity for $A C h R$ and rapsyn (D, arrow). Extrasynaptic AChR co-lacalized with rapsyn adjacent to the NMJ in the membranes of transfected fibers (E). In all tibialis anterior muscles, (both untreated and rapsyn-treated) of EAMG rats many intracellular AChR-rapsyn aggregates were found in proximity to endplates (F). Scale bars in A-E are $100 \mathrm{\mu m}$. Scale bar in $F$ is $50 \mu \mathrm{m}$. 
Because utrophin is involved in linking proteins of the NMJ to the cytoskeleton, we stained muscle sections of EAMG rats for utrophin, AChR and VAChT (results not shown). Similar to the loss of rapsyn and $A C h R$ also utrophin staining was reduced in endplates of tintreated EAMG muscles. In endplates that stained intensively for rapsyn and AChR in rapsyn-treated EAMG muscles, also the utrophin staining was increased. This indicates that utrophin contributes to the anchoring and stabilization of $\mathrm{ACh}$-rapsyn aggregates to the cytoskeleton.

We used two-photon confocal microscopy, at higher resolution, to examine the NMIs in more detail. Figure $5 \mathrm{~A}$ shows a normal endplate in an untreated control muscle (frontal view). In this orientation, the projection shows postsynaptic co-localization of $A C h R$ and rapsyn as well as adjacent presynaptic staining of VAChT. Figure $5 \mathrm{~B}$ shows an endplate in a rapsyn-transfected control muscle. No apparent structurall differences were observed between endplates from untreated and rapsyn-treated control muscles.

A representative endplate in an untreated EAMG muscle (Figure $5 \mathrm{C}$ ) shows a normal VAChT staining, but reduced amounts of rapsyn and $A C h R$. In the rapsyn-treated muscles most endplates were similar to untreated EAMG endplates, but additionally, in rapsyn-transfected fibers, endplates were found which stained intensely for rapsyn and AChR adjacent to VAChT (Figure 5D).

In order to analyze the efficacy of rapsyn-overexpression, the immunohistochemical staining of approximately 2000 endplates was quantified as a measure of postsynaptic $A C h R$ and rapsyn levels relative to the presynaptic VAChT levels. The endplates of the untreated EAMG muscles showed a significant decrease of AChR and rapsyn staining compared to endplates from untreated control muscles: The average AChR staining was reduced by $46 \pm 6 \%$ $(\mathrm{p}<0.001$, unpaired $\mathrm{t}$-test; Figure $6 \mathrm{~A}$ ) and rapsyn was affected even more severely and reduced by $64 \%( \pm 8 \% ; \mathrm{p}<0.001$, unpaired $\mathrm{t}$-test; Figure $6 \mathrm{C}$ ). Endplates in the rapsyn-treated chronic EAMG muscles had slightly increased average staining of rapsyn ( $36 \%, p=0.03$, paired t-test) and $\operatorname{AChR}(23 \% ; \mathrm{p}=0.29$, paired $\mathrm{t}$-test) compared to the contralateral untreated muscles. How ever, the rapsyn-overexpression did not increase the average synaptic AChR and rapsyn to normal levels. There was a weak negative correlation between AChR levels and antibody titer in the EAMG muscles, both for the untreated $\left(R^{2}=0.27 ; p=0.36\right)$ and the rapsyn-treated $\left(R^{2}=0.15\right.$, $\mathrm{p}=0.58$ ) muscles (Figure $6 \mathrm{~B}$ ), but a strong negative correlation between the synaptic rapsyn staining and the antibody concentration $\left(R^{2}=0.90 ; p=0.01\right.$ for untreated and $R^{2}=0.66 ; p=0.09$ for rapsyn-treated muscles, Figure 6D).

In EAMG rats, the analysis of immunohistochemical staining also revealed that at small, but significant proportion of endplates in the rapsyn-treated muscles contained more $A C h R(p<0.001)$ and rapsyn $(p<0.001, n=950$, unpaired $t$-test) than the endplates in contralateral muscles. Moreover, 135 out of 950 endplates (14\%) had a rapsyn concentration that was significantly higher (more than 2 standard deviations of untreated EAMG endplates) than in the contralateral EAMG muscles and 134 out of 950 endplates (14\%) also had significantly increased AChR concentration, suggesting that the increased rapsyn expression has helped to stabilize synaptic AChR concentration. Only $5 \%$ of the endplates in the untreated muscle sections had rapsyn and $A C h R$ concentrations reaching these levels. 


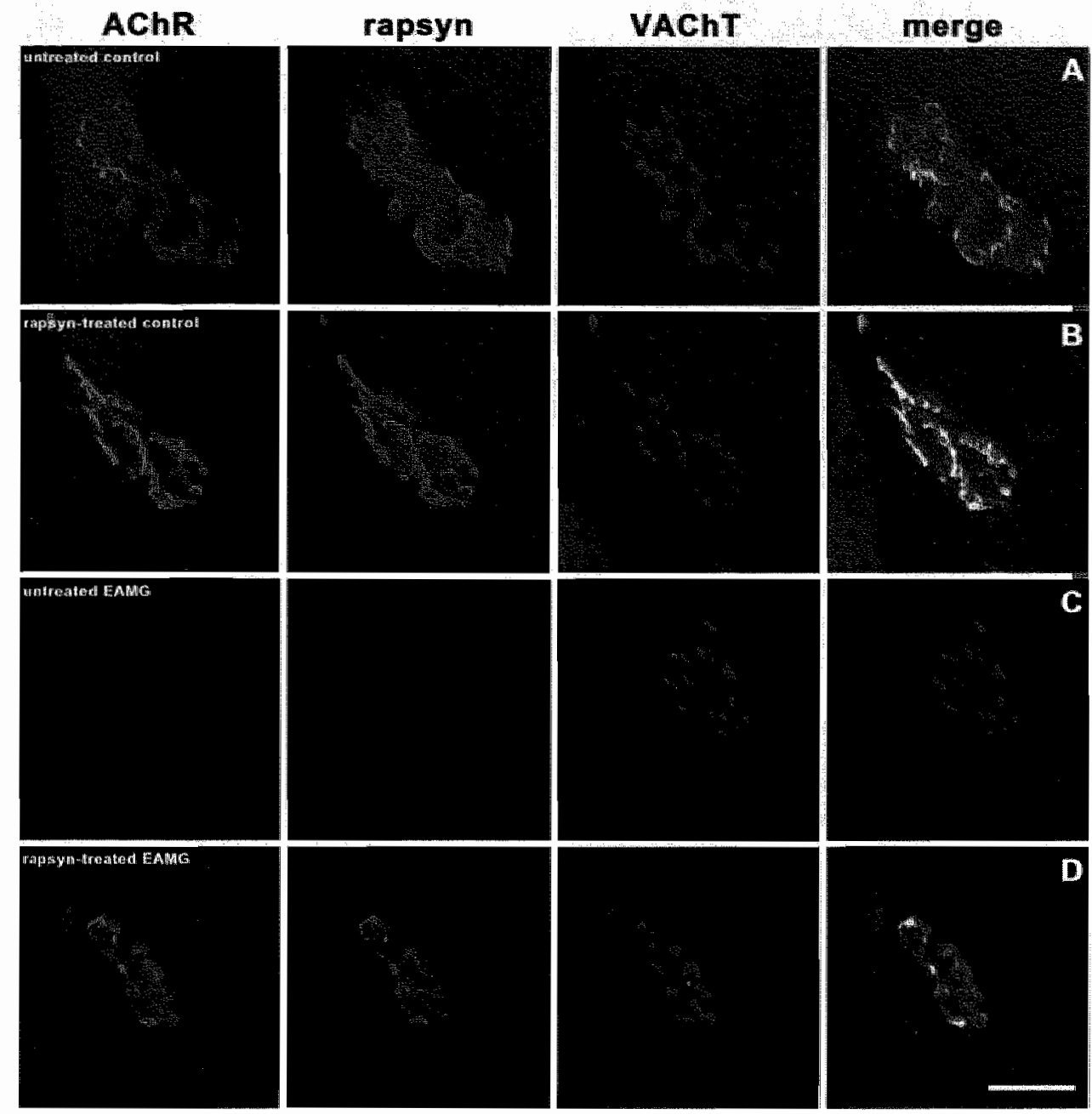

Figure 5. limmunohistocthemical analysis of the NMJ using two-photon laser scanning microscopy. AChR is stained red, rapsyn is stained green and WAChT is statned blue; merge on the right. A nomal endplate in an untreated control musicle shows co-localisation of AChR and rapsyn in the postsynaptic membrane and the adjacem presynaptic VAChT staining (A). Rapsyn-treated endplate of a control animal (B). Endplate in an untreated EAMG muscle with reduced staining of AChR and rapsyn (C). Transfected endplate in a rapsyn-treated EAMG muscle with increased AChR and rapsyn (D). Scale bar is $20 \mu \mathrm{m}$.

\section{Rapsyn overexpression does not prevent complement deposition at the neuromuscular junction}

At all endplates of tAChR-immunized animals the AChR co-localized with membrane attack complex (MAC) (not shown), consistent with a complement-dependent degradation of AChRs. Complement deposition was independent of rapsyn-tranfection. Therefore, the increased total $A C h R$ levels of rapsyn-transfected muscles can not be explained by reduced complement activation. In control muscles MAC staining was absent as expected. 


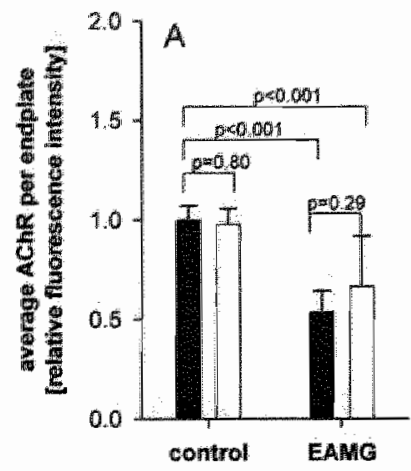

untreated fapsyn-treated

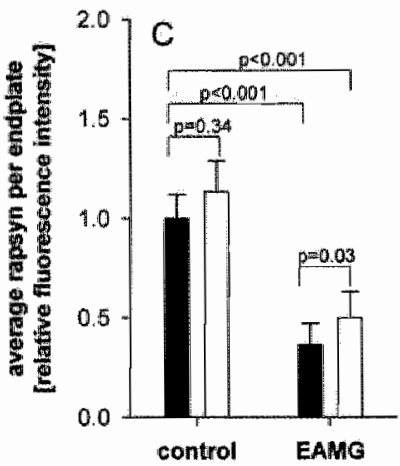

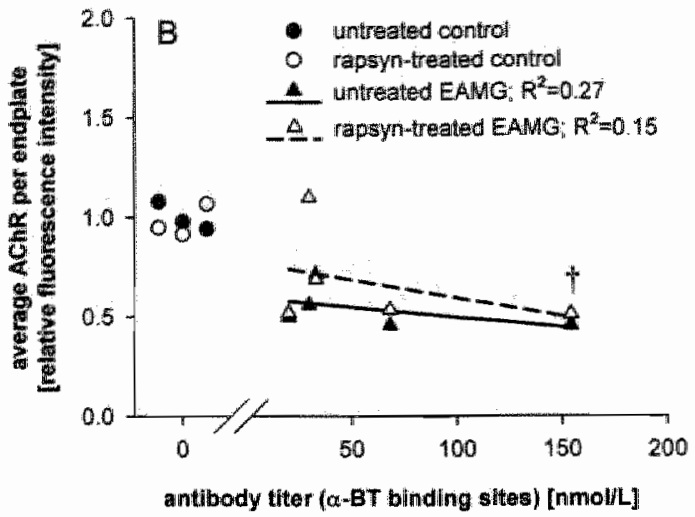

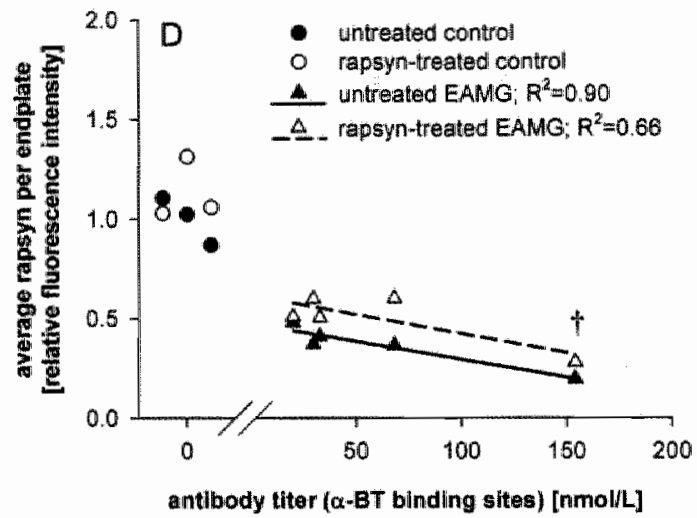

Figure 6. Measurement of fluorescence intensities of $A C h R$ (A and $B$ ) and rapsyn (C and D) relative to VAChT staining at 120 endplates per muscle. Staining intensities are normalized to the average intensities of untireated control undplates. The average staining intensities of endplates of identically treated muscles are shown in $A$ and $C_{y}$ and the average staining intensities in individual muscles are platted over the antibody titer in $B$ and $B$. The average rapsyn staining was significantly increased at endplates of EAMG animals (C), but this did not lead to a slignificant increase of average symaptic $A C h \mathbb{R}$ ( $A$ ) in the same animals. There was a strong negätive correlation between symaptic rapsyn concentration and antibody titer (D), but only a weak correlatilon for AChR and antibody titer. The data points below the cross belong to an animal with a clinical score of $+t$.

\section{Rapsyn overexpression does not improve neuromuscular transmission in EAMG rats}

To study if the increased amount of membrane AChR had an effect on the neuromuscular transmission, we measured the compound muscle action potential upon repetitive nerve stimulation in 3 control and 3. EAMG rats. In control rats, no decrement of the muscle compound action potential was found in untreated and rapsyn-treated muscles, even after injection of 3 or $6 \mu \mathrm{g}$ curare. In EAMG animals without curare injection, decrement was found only in the rapsyn-treated muscle of one rat. The contrallateral muscle 
Table 1. Morphometric analysis of endplates"

\begin{tabular}{|c|c|c|c|c|c|}
\hline & Regions Analysed & Nerwe Bouton Area [um'] & $\begin{array}{c}\text { Presynaptic Membrene } \\
\text { Length [um] }\end{array}$ & $\begin{array}{l}\text { Postiynaptic Nembrane } \\
\text { Length [an] }\end{array}$ & 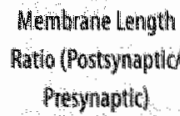 \\
\hline $\begin{array}{l}\text { untreated } \\
\text { contrat }\end{array}$ & 76 & $5.9 \pm 1.8$ & $5.8 \pm 1.8$ & $24.7 \pm 11.7$ & $45 \pm 0.9$ \\
\hline $\begin{array}{l}\text { rapsyn-treated } \\
\text { contral }\end{array}$ & 65 & $53 \pm 2.6$ & $4.9 \pm 1.3$ & $20.1 \pm 7.8$ & $4.0 \pm 0.3$ \\
\hline $\begin{array}{l}\text { Linfreated } \\
\text { EAMG }\end{array}$ & 57 & $5.0 \pm 2.3$ & $5.2 \pm 1.1$ & $12.7 \pm 3.0^{\circ}$ & $2.5 \pm 0.3^{6}$ \\
\hline $\begin{array}{c}\text { rapsyn-treated } \\
\text { EAMG }\end{array}$ & 24 & $6.9 \pm 4.6$ & $5.5 \pm 1.7$ & $8.1 \pm 2.2$ & $1.5 \pm 0.02$ \\
\hline
\end{tabular}

* Mean \pm SD of 3 muscles for each condition

a reglion here refers to an area of one synaptic bouton and the adjacent postsynaptic membrane

significantly different from untreated control endplates $(p<0.05)$ and significantly different from rapsyntreated EAMG endplates ( $\mathrm{p}<0.05)$

significantly different from all other conditions ( $p<0.05$ )

other differences in this table are not significant $(p>0.05)$

showed only decrement after injection of $3 \mu g$ curare. In the other two EAMG animals, decrement was induced bilaterally by injection of $3 \mu \mathrm{g}$ curare.

\section{Rapsyn overexpression reduces the postsynaptic membrane length in EAMG rats}

To study, therefore, how the rapsyn-overexpression affected the ultrastructure of the endplates in the rapsyn-treated EAMG muscles, we performed electron microscopic observations and morphometric analysis in the same 3 control and 3 EAMG rats. Rapsyn treatment did not appear to alter the structure of the neuromuscular junction in control muscles (Figure $7 \mathrm{~A}, \mathrm{~B}$ and Table 1) which showed normal postsynaptic folds in all examined regions ( 76 endplate regions of untreated control muscles and 65 regions of rapsyn-treated control muscles). The postsynaptic membrane was severely damaged in untreated muscles of EAMG rats (Figure $7 \mathrm{C}$ ) and even more so in the contralateral rapsyn-treated muscles (Figure 7D). In the untreated muscles of EAMG rats all 57 endplate regions analyzed had reduced postsynaptic folds or even a complete loss of postsynaptic folding. The average length of the postsynaptic membrane was significantly reduced compared to normal endplates (Table 1). In the contralateral rapsyn-treated EAMG muscles, all 24 regions analyzed showed a complete destruction of the postsynaptic folding. Moreover, fragmented subsynaptic nuclei (Figure 7F) were observed more frequently than in untreated EAMG endplates. The length of the postsynaptic membrane in rapsyn-treated endplates of EAMG rats was significantly shorter compared to the contralateral untreated muscles, or to control muscles $(\mathrm{p}<0.05$, Table 1$)$. 

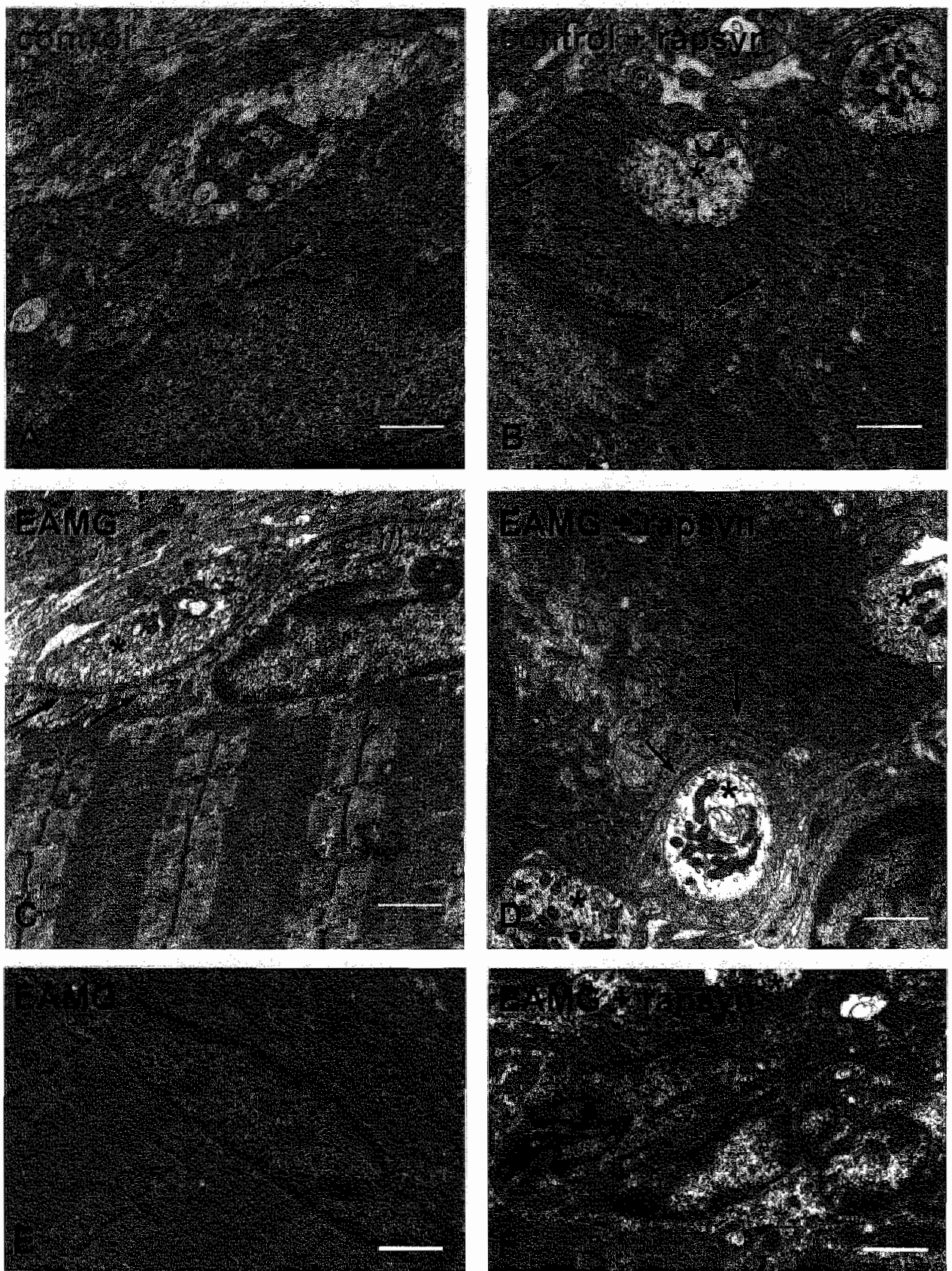

Figure 7. Electron microscopic examinations of the postsynaptic folds of motor endplates. NMJ in an untreated (A) and rapsyn-treated (B) tibialis anterior muscle. Damaged postsynaptic membrane without folds in an untreated tibialis anterior muscle (C) and in a rapsyn-treated tibialis anterior muscle (D) of an EAMG animal. Normal subsynaptic nucleus in an untreated EAMG muscle (E). Fragmented subsynaptic nucleus in a rapsymtreated EAMG muscle (F). Arrows indicate the postsynaptic membranes; stars indicate the nerve terminals; scale bars are I $\mu \mathrm{m}$. 


\section{Discussion}

Anti-AChR antibodies are the cause of the postsynaptic nembrane destruction in most myasthenia gravis patients, but the anti-AChR antibody titer is not the only factor determining disease severity. Previous observations suggested that intrinsic differences in the expression of postsynaptic proteins are correlated to resistance against EAMG (Hoedemaekers et al., 1998) and wt was demonstrated that transfecting the AChR anchoring protein rapsyn alone is sufficient to prevent disease symptoms in susceptible animals (Losen et al, 2005). Here, we investigated the effect of rapsyn transfection in ongoing chronic EAMG. The results show an increase of total membrane AChR in rapsyn-transfected muscles of chronic EAMG animals, in despite of high titers of ant $-\mathrm{ACl} \mathrm{R}$ antibodies. The increased $A C h R$ was localized mainly extrasynaptically, where it was bound in rapsyn-AChR-antibody aggregates. At the ultrastructural level, most postsynaptic regions in rapsyn-treated muscles showed increased damage of the postsynaptic membrane.

In rapsyn-treated muscles of control animals the amount of $A C h R$ was increased by $43 \%$ after two weeks, as measured by RIA of total muscle membrane extracts. The additional AChR was partly localized at endplates, as previously reported (Losen et al, 2005). Moreover, we observed extrasynaptic rapsyn-AChR aggregates in some fibers, which were not present in the contralateral untreated muscles. Rapsyn overexpression also increased the $A C h R$ levels in ongoing chronic EAMG. The AChR concentration in rapsyn-treated muscles was increased by $87 \%$ compared to the contralateral untreated muscles and was not significantly different from normal muscles. In rapsyn-treated muscles of EAMG rats, the AChR was found to be increased at NMTs of transfected fibers, but significantly increased $A C h R$ levels were only found in approximately $10 \%$ of the endplates. Confocal microscopy observations were consistent with these results showing that transfected fibers with high rapsyn expression near the endplate region, led to the normalization of $A C h \mathbb{R}$ levels. However, only a small number of endplates with normal AChR levels could be found. It is possible that these endplates were not yet completely destructed at the time of electroporation and were subsequently stabilized because of the rapsyn transfection. $\mathrm{A}$ high number of extrasynaptic AChR-rapsyn aggregates were also found in these fibers. Therefore, the extrasynaptic aggregates probably account for most of the increased AChRs in these muscles. Interestingly, a large proportion of the additional AChR was found to be bound to antibodies in vivo, suggesting that rapsyn mediated the transport of extrasynaptic intracellular AChR to the cell membrane. It is unlikely that this effect is caused by binding of circulating antibodies to epitopes of intracellular AChR because this phenomenon does not occur if isolated muscles are used for the muscle membrane extraction in contrast to total limb extraction (Verschuuren et al., 1992).

The analysis of immunohistochemical staining suggested that this extrasynaptic AChR

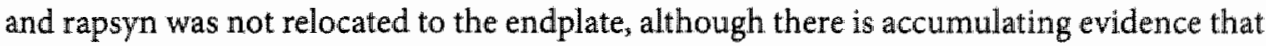
these proteins can be actively transported individually to the NMJ after ectopic expression in transfected or transgenic muscle fibers (Gensler et al, 2001; Gervasio and Phillips, 2005; Marangi et al., 2001; Ono et al., 2004). It is likely that extrasynaptic aggregates of AChR 
and rapsyn that are bound to autoantibodies can not be transported and this mechanism is responsible for the low levels of rapsyn and $A C h R$ observed at endplates of rapsyn-treated muscles and possibly also of untreated EAMG muscles. Trapping of AChR-rapsyn aggregates might aggravate the relatively low expression of rapsyn versus the AChR in EAMG (Asher et al., 1993). The possibility that impaired rapsyn trafficking plays a role in EAMG is also supported by results of Kishi and colleagues (Kishi et al. 2005), who observed that rapsyn mRNA expression is only increased 1.6 times at the NMJ compared to other muscle areas, whereas other synaptic proteins reached up to 80 fold increased synaptic expression. Interestingly, the synaptic enrichment of rapsyn $m R N A$ is age dependant in rats (Moscoso et al., 1995), thus paralleling the age related resistance against EAMG (Hoedemaekers et al., 1998). Therefore, it will be interesting to study if the enhancement of synaptic rapsyn expression helps to stabilize the AChR in EAMG more efficiently compared to the rapsyn expression in the whole muscle fiber.

The quantitative analysis of the ultrastructure showed that the increase of AChR and rapsyn levels in the muscle membrane had a detrimental effect on the postsynaptic folding. Moreover, fragmented subsynaptic nuclei were found in rapsyn-treated EAMG muscles. Since we found an increased binding of antibodies to AChR in rapsyn-transfected muscles, it is possible that an increased complement induced degradation of the postsynaptic membrane caused this effect. This support the hypothesis that rapsyn increases the clustering of $A C h R$ in the synaptic and extrasynaptic membrane and thereby making the $A C h R$ accessible to auto-antibodies and inhibiting its trafficking to the NMI from perisynaptic areas. It has been suggested that the reduced $A C h R$ density after passive transfer EAMG limits further damage to the postsynaptic membrane after subsequent immunization with anti-AChR antibodies (Corey et al., 1987). Our results are compatible with this mechanism and indicate that increased expression of $A C h R$ and rapsyn in ongoing EAMG enhances antibody binding and membrane damage. Consequently, rapsyn alone is not sufficient to anchor the AChR efficiently in the membrane when the neuromuscular junction is already damaged. We observed that apart from the AChR and rapsyn also utrophin levels are reduced in chronic EAMG. Loss of utrophin has also been reported for endplates of $\mathrm{MG}$ patients with anti-AChR antibodies (Ito et al., 1996; Slater at al, 1997). The loss of utrophin at the NMJ might restrict the anchoring of AChR-rapsyn clusters to the cytoskeleton. The difficulty to restore the NMI structure is illustrated by the fact that in passive transfer EAMG the postsynaptic membrane is still significantly reduced 54 days after injection of anti- $\mathrm{ACh}$ antibodies (Engel et al, 1979). In chronic EAMG, the postsynaptic membrane is continuously degraded and a large number of proteins have to be continuously replaced. Upregulation of rapsyn increases the AChR concentration in the disease, but this AChR is not sufficiently stabilized at the endplate. Therefore, a better understanding of rapsyn interaction with other proteins in the NMJ may reveal new factors that can modulate the clinical severity in myasthenia gravis. Taken together, our data help to understand the role of rapsyn in the disease and indicate that for a possible treatment of chronic EAMG muscles, rapsyn overexpression has to be targeted specifically to the endplate region and requires 
other postsynaptic proteins, including utrophin, for anchoring the AChR Moreover the results presented here show that rapsyn expression determines the susceptibillyty to EAMG and possibly MG in a complex way: high rapsyn expression anchors the AChR and prevents antibody and complement induced damage to the postsynaptic membrane but when the postsynaptic membrane is already damaged, high rapsyn expression increases membrane damage.

\section{Acknowledgements}

We are very grateful to Marc van Zandvoort and Wim Engels (Department of Biophysics, Universiteit Maastricht, The Netherlands) for their help with two photon confocal microscopy. We would also like to thank Marjo van de Waarenburg, B. Machiels and H. P. J. Steinbusch for their excellent technical assistance. This work was supported by grants from the Prinses Beatrix Fonds (MAR03-0115), LAssociation Française contre les Myopathies and the project grant (QLG3-CT-2001-00225) of the European Community program "quality of life and management of living resources". The BioRad TPLSM was obtained by grant no. 902-16-276 from the Medical Section of the Dutch Scientific Organization (NWO).

\section{References}

Asher O, Kues WA, Witzemann V, Tzartos SI, Fuchs S, Souroujon MC. Increased gene expression of acetylcholine receptor and myogenic factors in passively transferred experimental autoimmune myasthenia gravis. I Immunol 1993; 151: 6442-50.

Bloch RJ, Froehner SC. "The relationship of the postsymaptic $43 \mathrm{~K}$ protein to acetylcholine receptors in receptor clusters isolated from cultured rat myotubes. J Cell Biol 1987; 104: 645 -54 .

Corey AL, Richman DP, Agius MA, Wollmann RL. Refractoriness to a second episode of experimental nyyasthenia gravis. Correlation with AChR concentration and morphologic appearance of the postsynaptic membrane. J Immunol 1987; 138: 3269-75.

De Baets M, Stassen MH. The role of antibodies in myasthenila gravis. I Neurol Sci 2002; 202: 5i-11.

Engel AG, Sakakibara H, Sahashi K, Lindstrom IM, Lambert EH, Lennon WA. Passively transferred experimental autoimmune myasthenia gravis. Sequential and quantitative study of the motor end-plate fine structure and ultrastructural localization of immune complexes (IgG and C3), and of the acetylcholine receptor. Neurology 1979; 29:179-88.

Engel AG, Tsujihata $M$, Lambert $\mathrm{EH}_{0}$ Lindstrom JM, Lennon VA. Experimental autoimmune myasthenia gravis: a sequential and quantitative study of the neuromuscular junction uiltrastructure and electrophysiologic correlations. I Neuropathol Exp Neural 1976a; 35: 569-87.

Engel $A G$, T'sujihata M, Lindstrom JM, Lennon VA. The motor end plate in myasthenia gravis and in experimental autoimmune myasthenia gravis. A quantitative ultrastructural study. Ann N Y Acad Sei 1976b; 274: $60-79$.

Gautam M, Noakes PG, Mudd J. Nichol M, Chu GC, Sanes JR, et al. Failure of postsynaptic specialization to develop at neuromuscular junctions of rapsyn-deficient mice. Nature 1995; 377: 232-6. 
Genter S, Sander A, Komgrem $A$, Traina $G$, Giese $G$, Witzemann V. Assembly and clustering of acetylcholine recptors containing GFP-Iagged epsilon or gamma subunitsu selective targeting to the neuromuscular junction in vivo. Eur Biochem $2001 ; 268 ; 2209-17$.

Gervasio OL, Phillips WD. Increased ratio of rapsyn to ACh receptior stabilizes postsynaptic receptors at the mouse neuromuscular synapse I Physhol $2005,562: 673-85$.

Glass D), Bowen DC, Stitt TN, Kadziejewski C, Bruno J, Ryan TE, et al. Agrin acts via a MuSK receptor complex. Cell 1996; $85: 513-23$.

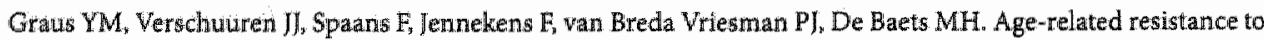
experimental autoimmune myasthenia gravis in tats. I Immunol 1993; 150; 4093-103.

Hoch W, MoConville $]_{*}$ Helms 5 , Newsom-Davis J, Melms A, Vincent A. Auto-antibodies to the receptor tyrosine Kinase MuSK in patients with myasthen id grawis without acetylcholine receptor antibodies. Nat Med 2001; 7: 365-8.

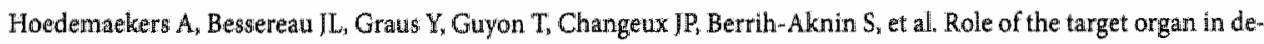
termining susceptibility to experimental autotmmune myasthenia gravis. J Neuroimmunol 1998; 89: 131-41.

Hoedemaekers A, Graus $Y$, Beijleveld L, van Breda Vriesman P, De Baets M. Macrophage infiltration at the neu. romuscular junction does not contribute to $\mathrm{AChR}$ loss and age-related resistance to EAMG. J Neuroimmunol $1997 \mathrm{a} ; 75 ; 147-55$

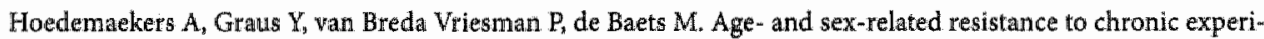
mental autoimmune myasthenia gravis (EAMG) in Brown Norway tats. Clin Exp Immunol 1997b: 107: $189-97$

Hoedemaekers A, Verschumren JJ, Spans F, Graus XF, Riemersma S, van Breda Vriesman PJ et al. Age-related susceptibility to experimental autoimmune myasthenia gravis: immunological and electrophysiological aspects. Muscle Nerve 1997c; 20: 1091-101.

Ito H, Yoshimura T, Satoh A, Takino H, Tsujthata M, Nagataki S. Immunohistochemücal study of utrophin and dystrophin at the motor end-plate in myasthenia gravis. Acta Neuropathol (Berl) 1996; 92: 14-8.

Kimura J. Electrodiagnosis in diseases of nerve and muscle: principles and practice. Oxford: Oxford University Press, 2001 .

Kishi M, Kunmer 'TT, Eglen SI, Sanes JR. LL5beta: a regulator of postsynaptic differentiation identified in a screen for symaptically enriched transcripts at the neuromuscular junction. / Cell Biol 2005; 169: 355-66.

Lennon VA, Lindstrom M, Seybold ME. Experimental autoimmune myasthenia: A madel of myasthenia gravis in rats and guinea pigs. I Exp Med 1975; 141: 1365-75.

Lindstron JM, Lennon VA, Seybold ME, Whittingham S. Experimental autoimmune myasthenia gravis and myasthenia gravis: biochemical and immunochemical aspects. Ann N Y Acad Sci 1976; 274: 254-74.

Losen M, Stassen MH, Martinez-Martinez P, Machiels BM, Duimel H, Frederik $\mathrm{P}_{\mathrm{i}}$ et al. Increased expression of rapsyn in muscles prevents acetylcholine receptor loss in experimental autoimmune myasthenia gravis. Brain 2005; $128: 232 \%-37$.

Marangi $P A$, Forsayeth IR, Mittaud $P_{s}$ Erb-Vogth $S_{3}$ Blake DJ, Monansard $M$, et al. Acetylcholine receptors are require for agrin-induced clustering of postsynaptic proteins. Embo J 2001; 20: 7060 73 .

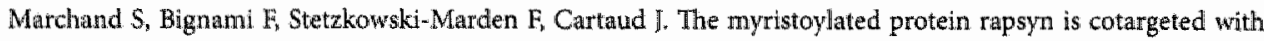
the ricotinic acefylcholine receptor to the postsynaptic menbrane via the exocytic pathway: I Neurosci $2000 ; 20: 521-8$.

Mir $\mathbb{L M}$, Bureau MF, Gehl I, Rangara R, Rouy D, Caillaud JM, et al. High-efficiency gene transfer into skeleta] muscle mediated by electic pulses. Proc Natl Acad Sci U S 1999; $96: 4262-7$. 


\section{Chapter 4}

\section{Experimental Autoimmune Myasthenia Gravis in Rhesus Monkeys}

Mario Losen and Marc H. De Baets 


\section{Introduction}

Myasthenia gravis (MG) is an antibody-mediated neurological disease affecting the neuromuscular junction. The understanding of the autoimmune disease MG has greatly profited from the corresponding animal model experimental autoimmune myasthenia gravis (EAMG). Here we will discuss the role of non-human primate studies in the development of therapies for the treatment of $M G$.

\section{The use of non-human primates in immunological research}

The phylogenetic proximity between higher primate species and humans is reflected by a high degree of immunological similarity (Bontrop et al., 1999; Bontrop et al., 1995). Nonhuman primates are therefore important experimental models for immune-based diseases in the human population. Non-human primate disease models are becoming important for the preclinical development and efficacy evalluation of new therapies, in particular those based on biological molecules, which by their high specificity cannot be tested in rodents.

The conditions under which non-human primates can be used in biomedical research are strictly regulated. The law on animal experimentation prescribes the qualifications of experimenters and the conditions under which experiments in primates can be considered. In general terms, non-human primates can only be used for in vivo experimentation where no alternative experimental possibilities exist, such as in vitro test systems, equivalent disease models in rats and mice or the patients themselves. Moreover, higher non-human primate species should not be used when the same information can be obtained in primate species that have a lower ranking on the evolutionary ladder.

An increasingly important area for non-human primate disease models is the safety and efficacy testing of biotechnology-based therapies (Bach et al, 1993; Jonker, 1990; Kennedy et al., 1997; Wierda et al., 2001). The minimal requirement to a valid animal model for safety-testing of a new drug is its sensitivity to the drug's specific pharmacological functions. As most biological therapies are only reactive in primates, non-human primates provide the most useful disease models for this purpose. The phylogenetic proximity between humans and non-human primates translates into a high degree of immunological similarity which is of importance for the research on fundamental patho-physiological processes in immune-based diseases.

In biological terms the species 'man' (Homo sapiens) together with the gorilla (Gorilla gorilla), chimpanzee (Pan troglodytes) and Bonobo (Pan paniscus) form the family of Hominidae. The evolutionary linkage of humans and chimpanzees, which is estimated at about 5 million years, is reflected by a high degree of genetic similarity; both species share $98.4 \%$ identity of their genome. This holds also true for highly polymorphic genomic regions, such as the major histocompatibility complex (MHC) and T-cell receptor (TCR) gene families (Bontrop et all, 1999; Bontrop et al, 1995). Chimpanzees are therefore a potentially important species as model of autoimmune diseases in the human population. Indeed, spondylo-arthropathies and neurological disorders in chimpanzees reminiscent of 
autommune disorders in the human population have been described (Alford and Satterfield, 1995; Rothschild and Woods, 1992; Rothschild and Woods, 1996). Ethical constraints, however, prohibit the induction of the experimental autoimmune disease models that are current in rodents and lower ranked primate species.

At present excellent autoimmune disease models exist in Old and New World monkeys. For the study of myasthenia gravis, experimentally induced disease models have been developed using rhesus monkeys (Macaca mulatta).

\section{Myasthenia gravis in humans}

$M G$ is a disorder of neuromuscular transmission, in most cases of an autoimmune or:gin with a few exceptions of genetic causes. $M G$ is characterised by muscle weakness and fatigue that can be restricted to the eye muscles, but generally affects all skeletal muscles. Initial symptoms comprise ocular motor disturbances, ptosis (drooping of the eyelids) or diplopia, oropharyngeal muscle weakness, difficulty chewing, swallowing, or taiking, and limb weakness (Drachman, 1994). The course of the disease is variable but usually progressive. Untreated MG can be lethal, e.g. when respiratory failure occurs. The autoantibodies in $85 \%$ of the patients are directed against the acetylcholine receptor $(\mathrm{AChR})$, whereas $10 \%$ have antibodies against the muscle-specific kinase (MuSK) (Hoch et al, 2001). "The cause of $\mathrm{MG}$ in a small percentage of patients is yet unknown. Currently available treatments for MG are reduction of the antibody titres in the patients' sera by immunosuppression and in. acute cases, by plasmapheresis or absorption of immunoglobulins using tryptophan-coated. collumns. However, immunosuppression has serious side effects. Plasmapheresis only improves the patients' conditions for a few weeks and requires intensive medical attendance. Hence, plasmapheresis is only indicated in a myasthenic crisis.

\section{Spontaneous autoimmune myasthenia gravis in animals}

MG spontaneously occurs in cats and dogs (Dau et al., 1979; Joseph et al., 1988; Shelton, 2002; Uchida et al ${ }_{*}$ 2002). Similar to the human disease, thymic abnormalities are seen and circulating anti-AChR antibodies can be detected by radioimmunoassay (Dau et al., 1979; Joseph et al., 1988). The immunological mechanisms are probably identical to human $M G$. To our knowledge, spontaneously occurring MG has not been described in non-human primates, which might be due to al low disease incidence.

\section{Experimental autoimmune myasthenia gravis}

The first convincing data suggesting that $M G$ has an autoimmune cause were reported by Patrick and Lindstrom in 1973, who he found that immunisation with $\mathrm{AChR}$ from electric eel (Electrophorus electricus) or pacific electric ray (Torpedo californica) evoked MG-like symptoms in rabbits (Patrick and Lindstrom, 1973). This model was named "experimen- 
tal atuoimmune myasthenia gravis" (EAMG). Since then, MG has been modelled in a a range of species, including guinea pigs, rats, mice, pigs and rhesus monkeys (Berman and Patrick, 1980, De Haes et al, 2003; Lennon et al, 1975; Tarrab-Hazdai et al, 1975; ToroGoyco et al, 1986). MG-like disease can be induced by antibodies which cross-react with the animals' own AChR. Anti-AChR antibodies damage the neuromuscular synapse by increased internalsation of the AChR (antigenic modulation) and by triggering complement activation. The antibodies alone are both essential and sufficient for the induction of EAMG symptoms. So, two EAMG models can be distinguished: (1) chronic EAMG, caused by immunisation with $A C h R$, as described above and (2) passive transfer EAMG, induced by injection of isolated anti-AChR antibodies.

\section{Chronic EAMG}

In chronic EAMG, many immunological aspects of $M G$ can be studied, such as AChR processing, APC presentation, the role of MHC, T-cell subsets and cytokines as well as many other issues in human $M G$ that are not directly related to the immune system. The availability of recombinant, mutant and transgenic mice strains offers the possibility to study the involvement of a wide range of proteins, including human imnunoglobulins (HuMAbMice) (Stassen et al., 2003). Susceptibility to EAMG depends strongly on the mouse strain used (Christadoss et al., 2000; Fuchs et al., 1976). In the most susceptible strains, 50-90\% of the animals develop disease symptoms, whereas others are completely resistant (Christadoss et al., 2000). Also, the severity of disease symptoms is variable. After immunisation of mice with $30 \mu \mathrm{g}$ Torpedo $A C h R$ ( $t A C h R$ ), the anti-tAChR serum titres are variable, ranging from $1-5 \mathrm{mg} / \mathrm{mL}$ for most cases. The proportion of these antibodies cross-reacting with the mouse AChR was found to be 0.2-2 \% (Berman and Patrick, 1980).

\section{Chronic EAMG in monkeys}

The first reports on EAMG in non-human primates dates back to 1975 (Tarrab-Hazdai et al, 1975). Rhesus monkeys (Macaca mulatta) immunised with four doses of 100-200 $\mathrm{gg}$ tAChR in CFA administered intradermally with 3 week intervals were found to develop typical MG signs. Initially, they observed "fatigue, hypo-activity, anorexia and weight loss", and subsequently "extreme flaccid paralysis of the limbs and trunk, sinking of the head and severe difficulties in breathing" (Figure la).

Electromyography showed a decremental response of the compound muscle action potentials during repetitive nerve stimulation, confirming impaired neuromuscular transmission. This hallmark symptom is used for diagnosis of MG in humans (Drachman, 1994). After injection of $67 \mu \mathrm{g} / \mathrm{kg}$ of the acetylcholine-esterase inhibitor Neostigmine bromide, the symptoms disappeared within $10 \mathrm{~min}$ (Figure 1 b), but this improvement only lasted for about $4 \mathrm{~h}$. 

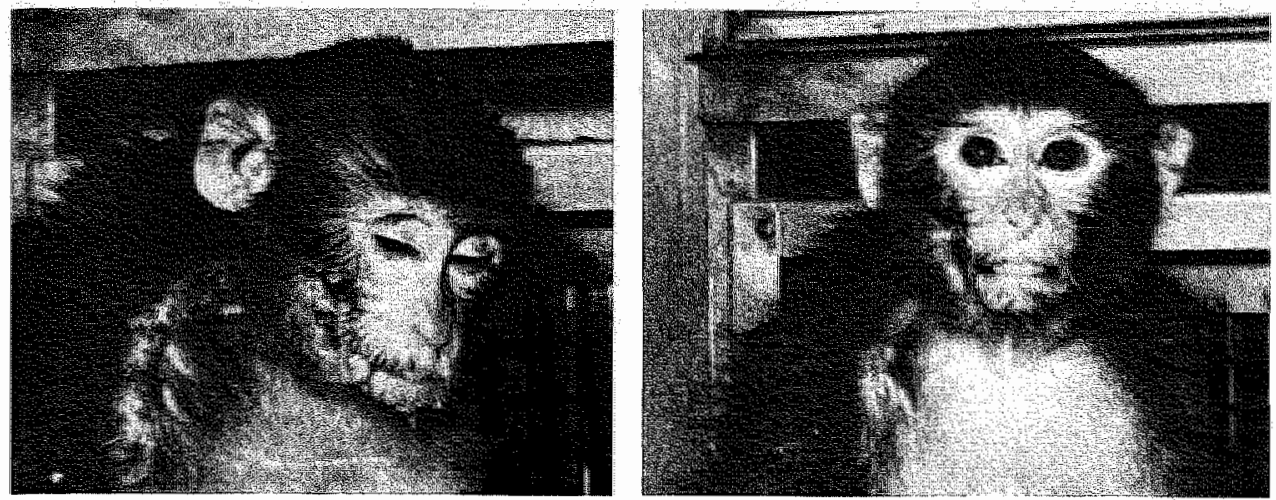

Figure 1. Experimental autoimmume myasthenia gravis in the rhesus monkey. Neositigmine bromide treatment of myasthenic monkey after active immunisation with Torpedo AChR. Left 5 min after injection off 67 $\mu \mathrm{g} / \mathrm{kg}$ neostigmine bromide (note the typical myasthenic drooping of the eyelids). Right: 10 min after injec tion of neostigmine bromide (Tarrab-Hazdai et al., courtesy of Sara Fuchs).

A similar study was performed by Toro-Goyco in 1986, in ten adult rhesus monkeys (Toro-Goyco et al., 1986). Four monkeys received a total dose of $240 \mu \mathrm{g}$ tAChR in CFA, divided into three equal doses with intervals of 14 days. Three monkeys developed severe clinical signs of MG and died of respiratory failure. One monkey died earlier without clinical signs, but was found to have anti-tAChR antibodies and muscle inflammation. Five animals that were immunised with a total dose of $60 \mu \mathrm{g}$ tAChR failed to develop EAMG signs, but anti-tAChR antibodies with titres up to $200 \mu \mathrm{g} / \mathrm{mL}$ were detected and persisted for more than one year. Antibody titres above $800 \mu \mathrm{g} / \mathrm{mL}$ were found to cause MG symptoms; three animals that died of EAMG had titres between 1 and $2 \mathrm{mg} / \mathrm{mL}$. These anti-tAChR antibody titres are very high compared to human $M G$ patient sera, where maximally $0.24 \mu \mathrm{g} / \mathrm{mL}$ are found (Somnier, 1993; Tindall et al., 1981), the total. IgG concentration in the serum beeing between 5 and $16 \mathrm{mg} / \mathrm{mL}$. However, the amount of antibodies cross-reacting with primate receptor was not determined in that study. Assuming that the degree of cross-reactivity is the same as observed in rodents, $(0.2-2 \%)$, the anti-primate $A C h R$ titre would be $4-40 \mu \mathrm{g} / \mathrm{mL}$.

The clinical observations clearly demonstrate the similarity of EAMG in rhesus monkeys and MG in humans. Active immunisation of non-human primates could again become rel evant when new MG therapies acting upstream of the antibody attack will become available.

\section{Passive transfer EAMG}

Toyka et al. have transferred purified IgG from MG patients to mice, which subsequently developed MG symptoms (Toyka et al., 1975). This passive transfer EAMG model is relevant for $M G$ in order to study the effector phase of the disease. Injection of antibodies against the main immunogenic region (MIR), or against the acetylcholine (ACh) binding sites of the AChR in experimental animals, induces EAMG within hours or days. "The source of antibodies can be serum of MG patients, serum from chronic EAMG animals or 
monoclonal antibodies (mAbs) produced in cell culture (Richman et al., 1980). Antibodies against the $\mathrm{ACh}$ binding sites induce a carare-like effect within 15-30 minutes (Balass et al, 1993). These antibodies block the ligand-induced opening of the ion channels.

Antibodies against the MIR of the human AChR are the most prominent cause of MG. In passive transfer EAMG, anti-MIR antibodies induce clinical signs within 8-48 hours depending on the dose and their affinity. The immunopathological mechanisms are antigenic modulation (Tzartos et al., 1985) and complement-mediated focal lysis of the postsynaptic membrane (Fazelkas et al., 1986; Lennon et al., 1978).

\section{Passive transfer EAMG in rhesus monkeys}

Transfer of IgG, purified from pooled. MG patients' sera, into rhesus monkeys ( $1 \mathrm{~g}$ IgG per $\mathrm{kg}$ body weight, on three consecutive days) leads to reversible MG symptoms (Heiniger and Toyka, personal communication). We are now in the process of characterising this model in order to be able to study therapeutic proteins in the future.

Two monkeys were injected with $0.8 \mathrm{~g} \mathrm{IgG}$ of pooled $\mathrm{MG}$ serum per $\mathrm{kg}$ of body weight on 3 consecutive days. In both monkeys a decrement of compound muscle action potential was found after repetitive nerve stimulation on day 8 (Table 1). Onlly one monkey developed clinical signs of MG starting on day 5 and lasting for one week. Serum titres against human AChR ranged from $22.8 \mu \mathrm{g} / \mathrm{mL}$ after last IgG injection on day 3 to $7.8 \mu \mathrm{g} / \mathrm{mL}$ on day 9 after the last injection.

Two more monkeys were injected with $0.5 \mathrm{mg} / \mathrm{kg}$ and $1 \mathrm{mg} / \mathrm{kg}$ of the recombinant antibody IgGL -637 , respectively, on 3 consecutive days. This human antibody binds to the human $\mathrm{AChR}$ specifically. It was selected as a Fab from a phage display library made from the thymus of a MG patient (Graus et al., 1997), and subsequently reconstructed to a full. IgGl. The antibody is produced in CHO cells as a fully human antibody, with some differences in glycosylation. Due to the IgGl properties the antibody is still pathogenic. The concentrations used are based on immunohistochemical staining of neuromuscular junctions in rhesus monkey muscle using serial dilutions of $\operatorname{lgG1-637}$ and MG patient sera.

None of the animals injected with IgG1-637 developed signs of MG but the monkey injected with the higher dose showed decrement on day 8 . This suggests that the antibody is actually binding to the rhesus AChR. In the future, higher doses of IgG1-637 will be tested to validate the pathogenic properties of the antibody.

Table 1. Effect of pasisive transfer EAMG in rhesus monkeys

\begin{tabular}{|c|c|c|c|}
\hline animal & antibody & dinical signs: & decrement \\
\hline 1 & pooled MG GgG:0.8 g//kg on 3 dlays & + & + \\
\hline 2 & pooled MG $\lg 6.0 .8 \mathrm{~g} / \mathrm{kg}$ on 3 days & - & + \\
\hline 3 & $\lg 61-6370.5 \mathrm{mg} / \mathrm{kg}$ on 3 days & - & - \\
\hline 4 & $1 \mathrm{~g} 61 \mathrm{um} 6371.0 \mathrm{mg} / \mathrm{kg}$ on 3 days & - & + \\
\hline
\end{tabular}




\section{Diagnosis and clinical management of EAMG}

Diagnosis of EAMG in rhesus monkeys can be performed much in the same way as in humans. (EA)MG can be diagnosed by: (1) Clinical presentation: wreakness of muscles after repetitive use, resulting in symptons such as ptosis, dysphagia, facial muscle weakness, generalised weakness and fatigue, weight loss and reluctance to move (Toro-Goyco et al. 1986); (2) analysis of anti-AChR and anti-MuSK antibodies by radioimmunoassay; (3) electromyography (EMG) showing a decremental response of the compound muscle action potential upon repetitive nerve stimulation (Pachner and Kantor, 1982) or single fibre EMG showing increased jitter (Verschuuren et al., 1990); (4) amelioration of symptoms following administration of acetylcholine esterase inhibitor (Neostigmine-test). In the chronic EAMG models for rhesus monkeys, immunisation with high doses of tAChR can be fatal due to respiratory failure. Neostigmine treatment was found unsuccessful in these cases (Toro-Goyco et al., 1986) and treatments with other drugs have not been reported.

Unlike in the chronic model, the MG symptoms in passively transferred EAMG are reversible. Mild symptoms can be treated with ACh esterase inhibitors. Occurrence of severe symptoms with respiratory problems can be avoided by carefully assessing antibody properties in vitro, using the abovementioned concentrations of polyclonal IgG from MG patient sera and $\operatorname{IgG1}-637$ as a reference.

\section{Myasthenia gravis therapies using the passive transfer EAMG model}

Generally, rhesus monkeys are used for effectivity testing of therapeutics that interact specifically with primate proteins. The humans and rhesus monkey AChR structure share a high sequence homology, but the alpha subunit of the human AChR contains an additional exon (MacLennan et al., 1993). Moreover, the character of pathogenic antibodies. in MG patients varies widely, ranging from the functional inhibition of $A C h$ binding to anti-MuSK antibodies. Thus, therapeutics tested in rhesus monkeys do not necessarily lead to the same results in (all) human MG patients.

At the level of the antibody attack, two approaches are possible to specifically prevent antibody binding to the AChR: (1) Absorbtion of anti-MIR antibodies. The efficacy of antibody adsorption was demonstrated in a passive transfer model with rhesus monkeys (Ferguson et al., 1995). They injected $10.5 \mu \mathrm{g} / \mathrm{kg}$ radioactive labelled $\mathrm{mAb} 35$, which is a rat anti-MIR antibody. This antibody could subsequently be depleted from the serum using a bi-specific reagent, which consisted of the tAChR crosslinked to an antibody against primate E complement receptor (anti-CR1). The $\mathrm{mAb} 35$ bound to the $\mathrm{ACh} R$-anti-CRI and was depleted from the circulation by the liver. A major risk of this system in a therapeutic application is the possible exacerbation of the autoimmune-response due to an additional response against the auto-antigen part of the bi-specific reagent. So far, this possibility has not been ruled out. (2) Protection of the MIR by antibody fragments (competition of antibodies for receptor). The alternative approach, using competitive antibody-fragments does not require $A C h R$ fragments and is therefore safer. A competitive recombinant antibody that 
protects the MIR is a promising agent to treat a myasthenic crisis. In a mouse model, Fabs have been demonstrated to protect the AChR against the intact antibodies (Papanastasiou et al., 2000; Toyka et al., 1980). However, these cannot be used for an application in patients, because rat antibody fragments will evoke a neutralising immune response. Moreover, Fabs antibody fragments have a short half life in vivo. A potential safety concern is that $F(a b)_{2}$ fragments are bivalent and still have some pathogenic potential by means of antigenic mod. ulation and increased internalisation of the AChR.

A protective agent should combine the following properties: high affinity for $A C h R$, long half-life, non-immunogenic, no complement activation and no cross-linking of AChRs. In order to obtain a molecule with these properties, we havecloned the human antibody IgG4-637 that does not activate complement. This recombinant antibody will be tested in vivo for its deficiency to cause EAMG. Since the IgG1-637 binds to primate AChR only, the passive transfer model with rhesus monkeys will be used. If this is successful, the next experiment will be the treatment of the disease with $\operatorname{IgG4-637}$ after injection of MG patient sera.

\section{References}

Alford $\mathrm{PL}$, Satterfield WC. Paralytic illiness resembling inflammatory polyradiculoneuropathy in a chimpanzee. I Am Vet Med Assoc 1995; 207: 83-5.

Bach JF, Fracchia GIN, Chatenoud L. Safety and efficacy of therapeutic monoclonal antibodies in clinical therapy. Irmmunol Today 1993; 14: 421-5.

Balass M, Heldman Y, Cabilly S, Giwol D, Katchalski-Katzir E, Fuchs S. Identification of a hexapeptide that mimics a conformation-dependent binding site of acetylcholine receptor by use of a plage-epitope library. Proc Natl Acad Sci U S A 1993; 90: 10638-42.

Berman PW, Patrick J. Experimental myasthenia gravis. A murine systern. J Exp Med 1980; 151: 204-23.

Bontrop RE, Otting $N$, de Groot $N G_{n}$ Doxiadis GG. Major listocompatibility complex class II polymorphisms in primates. Immunol Rev 1999; 167: 339-50.

Bontrop RE; Otting N, Sherendregt BL, Lanchbury IS. Evolution of major histocompatibility complex. polymorphisms and Th-cell receptor diversity in primates. Immunol Rev 1995; 143: 33-62.

Christados: P. Poussin M, Deng C. Animal models of myasthenia gravis. Clin Immunol 2000:94: $75-87$.

Dau $\mathrm{PC}$, Yano CS, Btinger S). Antibody to acetylcholine receptor in canine and human myasthenia gravis: differential cross-reactivity with human and rabbit receptor. Neurology 1979; 29: 1065-8.

De Haes A, Proost JH, De Baets MH, Stassen MH, Houwertjes MC, Wiercla JM. Pharmacokinetic pharmacodynamic modeling of rocuronium in case of a decreased number of acetylcholine receptors: a study in myasthenic pigs. Anesthesiology 2003; $98:$ 133-42.

Drachman DB. Myasthenia gravis. N Engl I Med 1994; 330: 1797-810.

Fazekas A, Komoly S, Bozsik B, Szobor A. Myasthenia gravis: demonstration of membrane attack complex in muscle end-plates. Clin Neuropathol 1986; 5: 78-83.

ferrguson PJ, Martin EN, Greene KL, Kulun S, Cafiso DS, Addona G, et all Antigen-based heteropolymers facilitate, via primate erythrocyte complement receptor type 1, rapid erythrocyte binding of an autoantibody and its clearance from the circulation in rhesus monkeys. I Immunol 1995; 155: 339-47. 
Fuchs $S$, Newo $D$, Tarrab-Hazdai $R$, Yar I. Strain diferences in the autommune response of mice to acetylcholine receptors. Nature $1976 ; 263: 329-30$.

Graus YT, de Baets MH, Parren PW Berrih-Aknin S, Wokke I, wan Breda Vresmail PI et al Humku anti-nicotinic acetylcholine receptor recombinant Fab fragments isolated from thymus-derived phage display libraries from myasthenia gravis patients reflect predominant specthities in serum and block the action of pathogenic serum antibodies. I Immunol 1997; 158: 1919-29.

Hoch W, McConville J, Helms S, Newsom-Davis J, Melms A. Vincent A Automantboties to the receptor tyrosine kinase MuSK in patients with myasthenia gravis without acetylicholine receptor antibodies. Nat Med 2001 ; $7: 365-8$

Jonker M. "The importance of non human primattes for predinical testing of immunosupprissive monodonal antibodies. Semin lmmunol 1990; $2: 427-36$.

Joseph R, Carrillo JM, Lennon VA. Myasthenia gravis in the cat, I Vet Intern Med 1988; 2: 75-9.

Kennedy $\mathrm{RC}_{\mathrm{y}}$ Shearer $\mathrm{MH}$, Hildebrand W. Nonhuman primate models to evaluate vaccine safaty and immunogenicity. Vaccine 1997; 15:903-8.

Lennon VA, Lindstrom 『M, Seybold ME. Experimental autoimmune myasthenia: A nodel of myasthenia gravis in rats and guinea pigs. I Exp Med 1975; 141: 1365 \% 75.

Lennon VA, Seybald $\mathrm{ME}_{\text {, }}$ indstrom IM, Cochrane $\mathrm{C}$, Ulevitch $\mathrm{R}$. Role of complement un the pathogenesis of experimentiat autoinmune myasthenia gravis. J Exp Med 1978; 147:973-83.

Maclennan $C$, Beeson $D$, Vincent $A$, Newsom-Davis $]$. Human nicotinic acetylcholine receptor alphansubunit isofornns: origins and expression. Nucleic Acids Res 1993; $21: 5463-7$.

Pachner $\mathrm{AR}_{3}$ Kantor FS. Nerve stimulation test in murine experimental autoimmune myasthenia gravis. Ann Neurol 1982; 11: $48-52$.

Papanastasiou D, Poulas K, Kokia A, Tzartos SI, Prevention of passively transferred experimental autoimmune myasthenia gravis by Fab tragments of monoclonal. antibodies directed against the main inmunogenic region of the acetylcholine receptor. ] Neuroimmunol 2000; 104: 124-32.

Patrick J, Lindstrom J. Autoimmune response to acetylcholine receptor. Science $1973 ; 180: 871-2$.

Richman DP, Gomez CM, Berman PW, Burres SA, Fitch FW, Arnazon BG. Monoclonal anti-acetylcholine receptor antibodies can cause experimental myasthenia. Nature $1980 ; 286: 738-9$.

Rothschild BM, Woods R. Spondyloarthropathy as an Old World phenomenon. Semin Artluritis Rheum 1992 ; $21: 306-16$.

Rothschild BM, Woods RJ. Inflammatory arthritis in Pongo. J Med Primatol 1996; 25: 414-8.

Shelton GD. Myastheria gravis and disorders of neuromuscular transmission. Wet Clin North Am Small Anim Pract 2002; 32: 189-206, vii.

Somnier FE. Clinical implementation of anti-acetylcholine receptor antibodies. I Neurol Neurosurg Psychially 1993: $56: 496-504$

Stasen MH, Meng F, Melgert E, Machiels BM, Im $5 H_{3}$ Fuchs $S$, et al Experimental autoimmune myasthenia gravis in mice expressing human immunoglobulin loci. I Nearoimmunol 2003; 135: 56-61.

Tarrab-Hazdai R, Aharonov A, Siman I, Fuchs $\$$, Abramsky $O$. Experimental autoimmune myasthenia induced in monkeys by purified acetylcholine receptor. Nature $1975 ; 256: 128-30$.

Tindall RS, Kent M, Wells L. A rapid immunoadsorbent adioimmunoassay for antimacetylcholine receptor antibody. I Immunol Methods 1981; $45: 1-14$. 


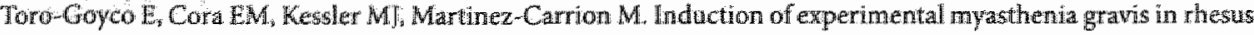
monkeys a model for the study of the human disease. P R Health Sci J 1986; 5: 13-8.

Toyka KV, Brachman DB, Pestronk $A, K a O$ L. Myasthenia gravis: passive transfer from man to mouse Science $1975: 190: 397-9$

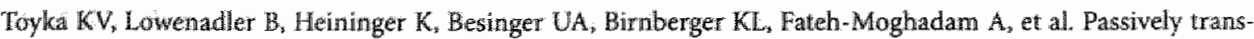
ferred myasthenia gravis: protection of mouse endplates by Fab fragments from human myasthenic IgG. I Neurol Newrosurg Psychiatry $1980,4,3: 836-40$

Tzartos $\$]_{3}$ sophianos $\mathrm{D}$, Efthimiadis $A$. Role of the main immunogenic tegion of acetylcholine receptor in my asthenia gravis. An Fab monoclonal antibody protects against antigenic modulation by human sera. J Immunol 1985; 134: 2343-9.

Uchida K, Awamura $Y$, Natcamura $T$, Yamaguchi $R$, Tateyama $S$. Thymoma and multiple thymic cysts in a dog with acguirred myasthenia gravis. J Vet Med Sci 2002; 64: 637-40.

Verschuuren JI, Spaans $\mathrm{F}$, De Baets MH. Single fiber electromyography in experimental autoinmune myasthenia grawis. Muscle Nerwe 1990; 13: 485-92.

Wierda D, Smith HW Zwickl CM. Immunogenicity of biopharmaceuticals in laboratory animals. Toxicology $2001 ; 158: 71-4$ 


\section{Chapter 5}

\section{Passive transfer of a human IgG4 anti-AChR antibody}

prevents myasthenia gravis in rhesus monkeys

Mario Losen, Pilar Martfnez-Martínez, Bert A. 't Hart, Herbert P.M. Brok, Frank Spaans,

Hans Duimel, Peter Frederik, Jan J.G.M. Verschuaren, Gert van Dijk, Klaus V. Toyka,

Efrosini Fostieri, Socrates J. Tzartos, Sonia Berrih-Aknin, Eugène Bosmans, Mischa Houtkamp, Luus Wiegman, Tom Vink, Ywo Graus, Janine Schuurman, Paul W.H.I. Parren and Marc H. De Baets. 


\section{Abstract}

Auto-antibodies against the nicotinic acetylcholine receptor of the skeletal muscle are the prime cause of myasthenia gravis. The human antigen fragment Fab 637, which binds specifically to the human acetylcholine receptor (AChR), protects against loss of AChRs by competitively blocking the binding of pathogenic myasthenia gravis autoantibodies in witro. Fab 637 was used for the recombinant production and functional analysis of whole human IgG1 637 and IgG4 637 which both bind to the human AChR with high affinity. lmmunohistochemical studies showed that the antibodies cross-reacted with muscle AChR of rhesus monkeys, but not of lower primates. IgGI 637 bound and activated complement, whiereas IgG4 637 did not. Both recombinant antibodies were bivalent and induced degradation of AChR of TE671 cells by antigenic modulation. The pathogenicity of the recombinant antibodies was tested in a passive transfer model in rhesus monkeys. Doses of 5 and $15 \mathrm{mg} / \mathrm{kg}$ of IgG1 637 induced muscle weakness and decrement of the muscle compound action potential upon repetitive nerve stimulation. IgG4 637 , in contrast, did not have any pathogenic effect when injected at the dose of $15 \mathrm{mg} / \mathrm{kg}$ suggesting that complement binding and activation is essential for induction of the disease. We therefore tested whether the IgG4 637 anti-AChR antibody could protect against the disease. When monkeys received the non-pathogenic IgG4 637 prior to challenge with the myasthenia-gravis-inducing IgG1 637, the induction of both muscle weakness and clinical symptoms was completely prevented. The results indicate that $\operatorname{IgGl}$ anti-AChR antibodies are sufficient to induce muscle weakness and IgG4 can prevent the pathogenic effects of IgGI autoantibodies in non human primates. "These studies suggest a novel approach for the development of a treatment of patients suffering from myasthenia gravis.

\section{Introduction}

Myasthenia gravis (MG) is an autoimmune disease characterized by muscular weakness and fatigue. The most common cause of $M G$ is the presence of auto-antibodies against the nicotinic acetylcholine receptor (AChR) of the skeletal muscle. Muscle $A C h R$ form an ion channel with five subunits, $\alpha 1, \gamma$ (replaced by $\varepsilon$ in adult), $\alpha 1, \delta$ and $\beta 1$, located at high density in the postsymaptic membrane of the neuromuscullar junction (NM)) (Fertuck and Salpeter, 1976; Gu and Hall, 1988). Anti-AChR auto-antibodies induce a loss of AChR, leading to an impaired neuromuscular transmission. The AChR loss can be demonstrated by low-frequency repetitive nerve stimulation (RNS) resulting in a decremental response of the compound muscle action potential (CMAP) (Oh, 1988).

Anti-AChR antibodies of all IgG subclasses have been found in MG patients, but IgG1 is the only isotype that is always present (Rodgaard et al., 1987). Pathogenic mechanisms may indude damaging the NMJ by focal lysis of the postsynaptic membrane due to complement activation, cross-linking of AChRs, leading to increased degradation and internalization of receptors ('antigenic modulation') (Heinemann et al., 1977; Kao and Drachman, 1977), inhibition of or ion channel function after prior stimulation (Bufler 
et al, 1996; Lang et al., 1988) and blocking of the ACh binding site (Almon et al.s 1974; Gomez and Richman, 1983). The extracellular domain of the al subunit contains the main immunogenic region (MIR), to which a major part of the pathogenic EAMG antibodies and possibly also a major part of MG auto-antibodies is directed (Tzartos et al., 1991; Tzartos and Lindstrom, 1980; Tzartos et al., 1982). Anti-MIR antibodies are also very effective in antigenic modulation.

The effects of auto-antibodies directed to the proteins of the NM) can be studied in an animal model of $\mathrm{MG}$, termed experimental autoimmune myasthenia gravis (EAMG). In the original experimental model, which led to the discovery of the cause of $M G$, rabbits were immunized with the AChR from the electric organ of Electrophorus electricus (chronic EAMG) (Patrick and Lindstrom, 1973). Subsequently, the similarity to human MG was demonstrated by immunising rhesus monkeys (Macaca mulatta) with repeated doses of Torpedo calfornica AChR (Tarrab-Hazdai et al., 1975). In a rodent chronic EAMG model, a sub-population of antibodies against the Torpedo or Electrophorus AChR that cross-react with the AChR of immunized animal were identified as the cause of the damage to the $\mathrm{NMJ}$, leading to a chronic disease (Lindstrom et al, 1976b). Sera from $\mathrm{MG}$ patients (Toyka et al., 1975; Toyka et al., 1977), AChR-immunized animais (Lindstrom et al., 1976a) and monoclonal anti-AChR antibodies (Tzartos and Lindstrom, 1980) have been shown to induce $M G$ symptoms for days to weeks when injected in naive animals (passive transfer EAMG).

Several antigen specific immunotherapy approaches have been tested in EAMG models, including anti-idiotypic Abs (Souroujon et al., 1986; Verschuuren et al, 1991), a competitor peptide that binds to a pathogenic anti-AChR mAb (Luo et al, 2001), induction of tolerance by oral administration of AChR (Wang et al., 1993) or AChR-derived synthetic peptides (Im et al., 2001), and elimination of AChR-specific T cells by genetically engineered antigen-presenting cells (Wu et al., 2001). To date, none of these therapeutic approaches have led to an efficient and feasible MG therapy. An alternative approach may be the direct blocking of the auto-antibody bindling sites on the AChR. The ideal blocking molecule should have a high affinity to the MIR of the AChR, without crosslinking and in activating the AChR nor activating complement. These criteria are met by antigen binding fragments (Fabs) as obtained from digestion of anti-AChR antibodies, and have been successfully used to prevent passive transfer EAMG in rats and mice (Loutrari et al, 1992; Papanastasiou et al., 2000; Richman et al., 1998; Toyka et al., 1980). Human anti-human AChR Fabs have been cloned and their ability to block MG serum Abs explored in recent years (Farrar et al., 1997; Fostieri et al., 2005; Graus et al., 1997; Protopapadakis et al., 2005; Rey et al., 2000; Stassen et al., 2003b; Zeidel et al., 1995). The MG patient-derived, anti-MIR Fab 637 protects against loss of AChR by MG sera in vitro, without interference with ion channel function (Graus et al., 1997). However, the short half life of monovalent Fab mo].ecules makes it unsuitable for treatment of patients. For the use in humans, the properties of the IgG4 antibody isotype fulfill the aforementioned prerequisites combined with a high 
in vivo stability. IgG4 has a half life similar to $\operatorname{lgGl}$, does not activate complement and is functionally monovalent in wivo (Aaberse and Schuurman, 2002; Schuurman et al., 1999). However, recombinant IgG4 produced in vitro is bivalent (Schuurman et al., 1999) and can potentially cause antigenic modulation. The role of IgG4 in the immune system is not fully understood. IgG4 antibodies may inhibit the inflammatory effects of other antibodies after frequent antigen exposure, for example during desensitization with allergens such as dust mite proteins (Nouri-Aria et al., 2004), immunity against phospholipase A2 after repeated bee stings (Aalberse et al, 1983, Nakagawa and Miyamoto, 1985) or in parasite infections (Garraud al., 2003; Vercelli et al., 1998). In a few cases IgG4 autoantibodies have been associated with autoimmune diseases, such as pemphigus (Rauterberg et al., 1988; Rock et al., 1989) autoimmune pancreatitis (Kamisawa et al., 2003) and MG with antibodies against the muscle specific kinase (MuSK) (Hoch et al, 2001). IgG4 anti-AChR antibodies have also been found in $40 \%$ of MG patients, but the IgG4 titers were one order of magnitude lower than those of IgG1, and IgG4 levels did not have a significant correlation with the severity of the disease (Rodgaard et al., 1987). The human IgG1 isotype is widely accepted as the most effective at recruiting the immune system against pathogens and to cause most pathogenic effects in antibody mediated autoimmune diseases, via the same mechanisms. This is because the Fc region of IgG1 interacts strongly with all types of FcyR on lymphoid and myeloid effectors and is a strong complement activator (Cragg et al., 1999; van Dijk and van de Winkel, 2001). Antibodies of the IgG4 isotype, which interact weakly with these receptors, are generally considered to have a neutralizing function and do not recruit immune effectors, thereby reducing antibody-mediated hypersensitivity reactions.

In this study we produced the human IgGl 637 and IgG4 637 isotypes from Fab 637 and analysed their properties both in witro and in vivo. The affinity for the AChR and the ability to bind complement and to cause antigenic modulation was measured in vitro. To test the hypothesized pathogenicity of $\operatorname{IgG1} 637$ and the neutralizing function of IgG4 637 in vivo, we used a passive transfer MG model with rhesus monkeys, because the antibodies based on the human Fab 637 solely cross-react with macaque AChR. The experiments are the first step towards the development of an antigen-specific IgG4 immunotherapy in MG patients.

\section{Methods}

Cloning and production of antibodies. IgGI 637: The light chain and the Fd coding sequences of Fab 637 (Graus et al., 1997) were cloned from the pComb3 bacterial expression vector to the mammalian vector pIgGl, which contains the sequence for the constant regions of human IgGl (Stassen et al, 2003a). First, the Fd sequence was cloned using Xhol and BstEII and subsequently XbaI and SacI were used for the light chain sequence. In pIgG1-637, the expression of each chain was regulated by a human cytomegalovirus promoter/enhancer (hCMV P/E) element, the secretion of the $H$ chain by mouse $H$ chain leader peptide and

the secretion of the L chain by a mouse k leader peptide. IgGI 637 flag. A flag tag was added to the carboxy-terminus of the IgG1 637 heavy chain for competition experiments. A frag- 
ment of 3268 bp containing the stop codon of the HC was subcloned from plgG1-637 to pBluescribe IIKS+ (Stratagene) using XhoI and Sall resulting in the plasmid pBS-HC. A Nhell restriction site replacing the stop codon was inserted by mutagenesis using the priner" ON-Nhel-mutm 5'-CTC TCC CTG TCT CCG GGT AAG CTA GCG CGA CGG CCG

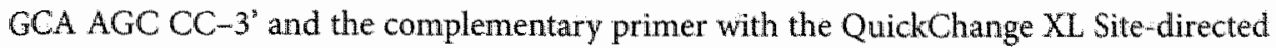
Mutagenesis Kit (Stratagene, La Jolla, CA, USA), according to the manufacturer's manual, resulting in the vector $\mathrm{PBS}-\mathrm{HC}-\mathrm{Nhel}$. Subsequently, the region between the stop codon and the Sall site of $\mathrm{pBS}-\mathrm{HC}$ was amplified by PCR using the forward primer ON-NheI-Flag 5"AAA GCT AGC GGA CTA CAA GGA CGA CGA TGA CAA GTG AGT GCG ACG GCC GGC AAG-3' and the reverse primer 5"- ATA CCG TCG ACG CTC TCC Cl'T ATG $C-3$. The primer $\mathrm{ON}-\mathrm{Nh}$ - Flag added a Nhel site and the coding sequence of the flag tag (DYKDDDDK) followed by a stop codon to the PCR product. The fragment was purified and cloned into pBS-HC-NheI with Nhel and SalI resulting in the plasmid pBS-HC-NheIflag. Finally, the XhoI-SgrAI fragment from pBS-HC-NheI-flag was subcloned into pIgG1637. IgG4 637: The genomic constant region of human IgG4 was cloned into the pEE6.4 vector (Lonza Biologics) resulting in the vector pTomG4. The VH coding region of Fab 637 was amplified from pIgG1-637 by PCR, using the primers MGHCexfor 5 ' GAT AAG CTT GCC GCC ACC ATG GAA TGG AGC TGG GTC TTT CT 3' and MGHCexrev 5' GAT' CGT CTC GGG CCC TTG GTG GAG GCC GAT GAG 3; introducing suitable restriction sites for cloning into pTomG4 and an ideal Kozak sequence. The fragment was cloned using HindIII and Esp3I resulting in the plasmid pTomG4MG. The complete VH coding region of $\mathrm{p}$ TomG4MG was sequenced. The VL coding region of Fab 637 was amplified from plgG1-637 by PCR, using the primers MGLCexfor 5 ' GAT'AAG CTT GCC GCC ACC ATG GGT GIT CCC ACT CAG GTC CT 3' and RACElambdal $5^{\circ}$ CCA GTG TGG CCT TGT TGG CT 'T GAA G 3', introducing HindIII and AvrII restriction sites and an ideal Kozak sequence. The resulting fragment was cloned using these enzymes into the vector pConLam2 (Lonza Biologics), encoding the constant region of the human lambda light chain. A clone was isolated and the complete VL coding region was sequenced. The construct was named pConLamMG. Cell culture. CHO-KI (ATCC Number CCL-61) was cultured in HAM's F12 (Gibco/Invitrogen), supplemented with 10\% fetal bovine serum (Bodinco, Alkmaar, The Netherlands). Cells were transfected with Lipofectin (Gibco/Invitrogen), according to the manufacturer's manual. Transfected cells were adapted to suspension culture. A monoclonal stable cell line was obtained by selection with $50 \mu \mathrm{M}$ MSX and limiting dilution. The highest producer was selected for large-scale production. A 10-liter hollow fiber culture yielded $558 \mathrm{mg}$ protein G-purified IgG1 637. For the production of IgG4 637, pTomG4MG and pConlamMG were cotransfected into CHO-K1SV cells (Lonza Biologics) and stable clones were selected after selection with $50 \mu$ M MSX. A $5 \mathrm{~L}$ spinner batch culture yielded $900 \mathrm{mg}$, protein A purified, IgG4 637. For the transient production of IgG1 637, HEK 293 Freestyle cells (Invitrogen) were transiently transfected using 293Fectin (Invitrogen) with the plgG1-637 vector, according to the instructions of the manufacturer. The antibodies were purified using Prot $\mathrm{G}$ sepharose (for IgG1) or protein A (for IgG4). 
Antibody analysis. Human IgG in culture supernatants were measured by ELUSA. Microtiter plates (Microlon, Greiner) were coated over-night with goat $\mathbb{F}\left(a^{\prime}\right) 2$ anti human IgG FC (Cappel, 56961), blocked with 5\% non fat dry milk in PBS and incubated with serial dilutions of culture supernatant or purified antibodies. The human IgG was detected with goat anti-human IgG FC PO (Jackson ImmunoResearch, 109035008) and analysed with TMB $\left(3,3-5,5^{3}\right.$-tetra-methyl benzidine). For the detection of the Flag-tag, plates were coated and incubated with samples as above and analysed with mouse anti Flag M2 (Stratagene), biotinylated goat anti mouse (Jackson ImmunoReserach, 115065146), avidin-biotincomplex (avidin solution and peroxidase-biotin solution, both diluted 1:1000, Vectastain ABC-kit, vector) and TMB.

The antibodies used for the animal studies were evaluated for their purity, the presence of aggregates and appearance on SDS-PAGE. The IgGI and IgG4 637 antibodies used showed no unexpected products when evaluated on SDS-PAGE. Evaluation of the IgG1 637 on non-reducing SDS-PAGE showed the appearance of a major band of $170 \mathrm{kDa}$; evaluation of the IgG4 637 on non-reducing SDS-PAGE showed as expected, the presence of a major band of intact IgG (158 kDa) and of $\operatorname{IgG} 4$ half-molecules (73 kDa). Batches of IgG were tested in HP-SEC and showed to be least $94 \%$ monomeric. Endotoxin levels of all batches used were below $0.1 \mathrm{EU} / \mathrm{mg} \mathrm{IgG}$.

The complement binding of IgG1 637, IgG4 637 and of polyclonal isotype controls were tested by ELISA. Microtiter plates were coated overnight at room temperature (RT) with the test antibodies serially diluted from $10 \mu \mathrm{g} / \mathrm{mL}$ to $0.15 \mu \mathrm{g} / \mathrm{mL}$ in PBS. For the CIq ELISA, wells were blocked with C1q buffer (PBS supplemented with $0.1 \%$ w/v gelatin and $0.05 \% \mathrm{v} / \mathrm{v}$ Tween -20 ). This blocking buffer was also used for all subsequent washing and incubation steps. The wells were incubated with $2 \mu \mathrm{g} / \mathrm{mL}$ human C1q (Quidel, San Diego, CA, USA, A400) (100 $\mathrm{L} /$ well, $\left.37^{\circ} \mathrm{C}, 1 \mathrm{~h}\right)$, subsequently with rabbit anti-human

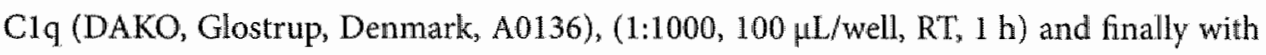
HRP-conjugated swine anti-rabbit IgG-Fe (DAKO P0399) diluted (1:2500, $100 \mu \mathrm{l} / \mathrm{well}$, $\mathrm{RT}, \mathbb{1} \mathrm{l}$ ). For the $\mathrm{C} 3 \mathrm{~b}$ and C4b ELISA's, the wells were blocked with PBS/BSA buffer (PBS supplemented with 1\% BSA). For all subsequent washing steps PBST [PBS supplemented with $0.05 \%(\mathrm{v} / \mathrm{v})$ tween-20] was used. Plates were incubated with pooled human serum dilluted $1 / 33(\mathrm{v} / \mathrm{v})$ in 'TBS buffer (15 mM Tris pH 7.45, $145 \mathrm{mM} \mathrm{NaCl}, 10 \mathrm{mM} \mathrm{CaCl} 2,5 \mathrm{mM}$ $\mathrm{MgCl} 2,0.2 \% \mathrm{BSA}_{x} 1 \mathrm{~h}$ at $37^{\circ} \mathrm{C}$ ) and subsequently with either mouse anti-human $\mathrm{C} 3 \mathrm{~b}$, (DAKO, clone HAV3-4, M0836), diluted in PBST/BSA (PBST supplemented with 0.1\% BSA), (1:100, $100 \mu \mathrm{L} /$ well, R"T, 1 h) or mouse anti-human C4b (Brunschwig, Basel, Switzerland, Hyb 162-02) diluted in PBST/BSA (1:2000, $100 \mu \mathrm{l} /$ well, RT, $1 \mathrm{~h}$ ). Wells were then incubated with HRP -conjugated rabbit anti-mouse IgG (Jackson Immuno Research, Westgrove, USA, 315-035-046), diluted in PBST with 0.1\% BSA (100 $\mu \mathrm{L} /$ well, 1 hr RT). All assays were developed with freshly prepared ABTS solution (1 mg/mL; ABTS: 2,2'-azinobis (3-ethylbenzthiazoline-6-sulfonic acid]) at RT in the dark for 30 minutes.

The competition of IgG4 637 and IgG1 637 with IgGI 637 flag was measured by radioimmunoassay using AChR from TE671 cell membrane extract. Approximately $2 \mathrm{nmol}$ 
of AChR was labeled with an excess of ${ }^{125} \mathrm{IBT}$ and incubated for $8 \mathrm{~h}$ at $4^{\circ} \mathrm{C}$ with 5 nmol IgGI 637 flag and different concentrations of $\mathrm{IgG1} 637$ or $\mathrm{IgG} 4637$ in a total volume of $75 \mu \mathrm{L}$. To each sample $2 \mu \mathrm{g}$ mouse anti-FLAG M2 (Sigma) and $1 \mu \mathrm{L}$ normal mouse serum (co-precipitant) were added and incubated at for another $12 \mathrm{~h}$. The mouse antibodies were precipitated with $100 \mu \mathrm{L}$ preabsorbed goat serum containing polyclonal goat anti mouse antibodies for $4 \mathrm{~h}$ at $4^{\circ} \mathrm{C}$. The preabsorbed goat serum was prepared by incubation with normal human serum $(1: 1(\mathrm{v} / \mathrm{v}))$ at $37^{\circ} \mathrm{C}$ for $2 \mathrm{~h}$ and cleared by centrifugation and filtersterilization. The samples were then centrifuged and processed as described above.

The specificity of IgG1 637, IgG1 637 flag and IgG4 637 was determined by radioimmunoassay (Lindstrom et al., 1981), using human AChR (prepared from the human TE671 rhabdomyosarcoma cell line (Luther et al., 1989)), Torpedo AChR (prepared from Torpedo californica) or hybrid AChR (human al subunit and Torpedo $\beta, \gamma$ and $\delta$ subunits Loutrari et al., 1997)). The AChR was labeled with ${ }^{125} I$-labeled $\alpha$-bungarotoxin (Amersham) and incubated with different sample concentrations and human serum as co-precipitant. Human IgG was precipitated by incubation with polyclonal goat anti-human Ig, for $4 \mathrm{~h}$ at $4^{\circ} \mathrm{C}$ and centrifugation at $15000 \mathrm{~g}$ for $10 \mathrm{~min}$. The pellets were washed twice with $0.5 \%$ Triton $\mathrm{X}-100$ PBS and measured in a $\gamma$-counter.

The binding of IgG1 637 to AChR expressed on TE671 or MITC cells (Wakkach et al., 1999) was measured by flow cytometry (FACSCalibur, Becton Dickinson Immunocytometry Systems, Mountain View, CA, USA). Trypsinized cells were incubated with sample antibody and subsequently writh FITC-conjugated goat anti-human Ig. "The signal was amplified by EAS Kit (Flow Amp Systems, Cleveland, USA). For detection of IgG1 637 binding, to cytoplasmic $\mathrm{AChR}$, cells were permeabilized with paraformaldehyde solution ( $2 \%$ ).

Antigenic modulation of AChR. The enhanced degradation of surface AChR of TE671 cells by antibodies was measured by incubating confluent cells with serial dilutions of IgG1 637, IgG4 637 and human intravenous immunoglobulin (IVIg) (Gammaquin; Sanquin, Amsterdam, The Netherlands). Cells were incubated for 3 hours with antibodies diluted in DMEM supplemented with $40 \mu \mathrm{M}$ cycloheximide. The remaining $A C h R$ was labeled with an excess of ${ }^{125}$ I-labeled a-bungarotoxin in the same medium without antibod. ies for one hour and washed 3 times with PBS. Nonspecific binding was measured by incubating cells with unlabelled $\alpha$-bungarotoxin prior to incubation with ${ }^{125} I$ - $\alpha$-bungarotoxin.

Passive transfer MG in rhesus monkeys. Animal experiments were performed at the Biomedical Primate Research Center (Rijswijk, "The Netherlands) with permission of the Committee on Animal. Welfare (DEC), according to Dutch governmental rules. Female Macaca mulatta monkeys between 3 and $4 \mathrm{~kg}$ were injected with 3 escalating doses of antibody on consecutive days. The effect of IgG1 637 was initially tested at a dose of $0.5 \mathrm{mg} /$ $\mathrm{kg} / \mathrm{day}$ and $1 \mathrm{mg} / \mathrm{kg} / \mathrm{day}$ and subsequently $1.7 \mathrm{mg} / \mathrm{kg} / \mathrm{day}$ and $5 \mathrm{mg} / \mathrm{kg} / \mathrm{day}$ (Table II). The effect of $\operatorname{lgG} 4637$ was tested alone or in combination with $\operatorname{IgG1} 637: 5 \mathrm{mg} / \mathrm{kg} / \mathrm{day}$ of IgG4637 or IVIg (Gammaquin) were injected in the mornings on 3 consecutive days (total dose $15 \mathrm{mg} / \mathrm{kg}$ ). Six hours after the first injection, animals received a second injection consisting of $1.7 \mathrm{mg} / \mathrm{kg} / \mathrm{day}$ IgGI 637 or IVIg on each of the three experimental days 
(5 mg/kg total dose). The IVIg did not contain ant antibodies against AChR and was only given to control for any events potentially related to the injection of human antibody. Blood samples were taken on different days and analyzed for anti-human $A C h R$ titer using the Acetylcholine Receptor Autoantibody RRA Kit (IBL, Hamburg, Germany). Each animal was used for an experiment only once to avoid the effect of a primate anti-human antibody response. In total 14 monkeys were used which all survived the experiments and were not sacrificed for analysis.

Electromyography. For EMG measurements, the monkeys were anesthetized with $10 \mathrm{mg} / \mathrm{kg}$ ketamine. Decrement of compound muscle action potential (CMAP) was measured in the extensor digitorum brevis upon stimulation of the peroneal nerve below the fibular head. Recording and stimulation were performed with surface electrodes. The reference ellectrode was placed distal to the active recording electrode at a distance of 3 to $4 \mathrm{~cm}$. Using single stimuli of $0.1 \mathrm{~ms}$ duration and gradulally increasing intensity, the current strength was determined at which a maximal CMAP amplitude was reached. To detect a decremental response, 10 stimuli with a 20 to $30 \%$ higher stimulus strength were given at 2, 3 and $5 \mathrm{~Hz}$. Test results had to be reproducible and were considered positive when both the amplitude and the area of the negative peak op the CMAP showed a decrement of at least $10 \%(\mathrm{Oh}, 1988)$.

Immunohistochemistry Biopsies of intercostal muscle were taken on day 7 after the first injection of human immunoglobulins. The biopsies were frozen immediately on melting isopentane and stored at $-80^{\circ} \mathrm{C}$. Muscle biopsies were cut at $10 \mu \mathrm{m}$ on a cryostat, fixated in acetone (10 min at RT), blocked for endogenous peroxidase and blocked for unspecific binding to Fc-receptors using 10\% normal goat serum. To visualize the IgG1 637 and IgG4 637 in the intercostal muscle of rhesus monkeys, cryosections were incubated with mouse antibodies directed against human IgGl allotype Gml(a) (HP6184) and against human IgG4 (MH164-4). These antibodies showed no cross-reactivity with endogenous IgG of rhesus monkey and hence gave a low background in immunohistochemistry on monkey tissue. Subsequently, sections were incubated with FITC conjugated goat-anti-mouse IgG (GaM IgG-FITC) (1:100; $30 \mathrm{~min}$ ) and peroxidase conjugated sheep-anti-FITC (Sh-antiFITC-PO) (1:100; $30 \mathrm{~min})$. Peroxidase activity was visualized with amino-ethyl-carbazole (AEC; red color) and nuclei were stained with hematoxylin (blue). To confirm the localization of AChR in the rhesus muscle end-plates, cryosections were stained with Alexa 495 conjugated bungarotoxin (1:300,30 min).

Electron microscopy. Intercostal muscle biopsy fragments of $3 \mathrm{~mm}$ diameter were submerged in fixation buffer ( $2.5 \%$ glutaraldehyde in $0.1 \mathrm{M}$ phosphate buffer $\mathrm{pH}=7.4$ ) and postfixed with $1 \%$ osmiumtetroxide in $0.1 \mathrm{M}$ phosphate buffer, $\mathrm{pH} 7.4$, dehydrated through a graded ethanol series and embedded in epoxy resin (Glycid ether 100, Serva, Heidelberg, Germany). Endplates were located in toluidine blue-stained semi-thin sections. Ultra-thin sections from selected areas were contrasted with uranyl acetate and lead citrate and analyzed with a Philips CM 100 electron microscope. 


\section{Results}

\section{Production of human anti-AChR antibodies of the $\lg \mathrm{G} 1$ and $\lg \mathrm{G} 4$ subclasses.}

Fab 637 is an antibody Fab fragment binding to the main immunogenic region (MTR) of the human $A C h R$, which was previously isolated from a phage display library prepared from the thymus of an MG patient (Graus et al., 1997). For the production of the corresponding whole antibodies IgG1 637 and IgG4 637, the coding sequences for Fab 637 were subcloned from the original bacterial $\mathrm{pComb3} \mathrm{H}$ expression vector into mammalian expression vectors containing the coding sequences for human IgG1 and IgG4 constant heavy chain regions. These vectors were then transfected into CHO or HEK 293 cells leading to the secretion of the antibodies to the culture medium. In addition, the recombinant antibody IgGI 637 flag was produced, in which a Flag-tag was added to the C termini of the IgG1 637 heavy chains.

\section{The MG autoantibody IgG1 637 binds specifically to the AChR a1-subunit of human and ma- caque muscle and to the AChR of human myoid cells.}

To determine whether the specificity of IgGl 637 was the same as its parental Fab 637, several tests were performed (summarized in Table I):

Table I. Specificity of IgG1 637

\begin{tabular}{|c|c|}
\hline AChR source & Ig 61637 binding \\
\hline Forpedio AChR (Lindstrom et al., 1981) & $-($ RIA, Fïgure 1D) \\
\hline human AChR peptide a1-210 (Psaridi-Linardaki et all.2002) & - (ARA, Figure 1B) \\
\hline humban a Torpedo BV AChR (Loutrari et al., 1997) & $+\left(R \mid A_{v}\right.$ Figure $\left.1 C\right)$ \\
\hline human fetal AChis from IE extract (Luther et al., 1989 ) & $+\left(R i A_{0}\right.$ Figure $1 A$ and 3$)$ \\
\hline human fetal AChe in fe cells (Luther et al, 1989 ) & $+\left(\right.$ FACS, Figune $2 A_{r} B$ and $\left.C\right)$ \\
\hline human AChR in MIC cells (Wakkach et al. ${ }_{.,}$1999) & $+\left(\mathrm{FACS}_{\mathrm{s}}\right.$ Figure 2D) \\
\hline adult AChR in human endplate & $+(\mathrm{HC}$, not shown) \\
\hline addill AChR in Macaco mulotra endplate & $+(1 H C$, Figulire B) \\
\hline adult AChR in Macaca fascicularis endplate & $+(1 \mathrm{HC})$ (Graus et a \\
\hline adult AChR in Cawith rong jochus endplate & $-[\mathrm{HO}]$ \\
\hline rat AChR & - (RIA] (Graus et all, 1997) \\
\hline
\end{tabular}

The specificity of Fab 637 for the human a subunit of the AChR was preserved in the full size IgG1 637: It bound to recombinant human/Torpedo hybrid AChR (Figure 1C), with the human $a 1$ and the Torpedo $\beta \gamma \delta$ subunits, but did not bind to the wildtype Torpedo AChR (Figure 1D). IgG1 637 also did not bind to the recombinant human al-210 peptide (Figure 1B), confirming the specificity for a conformational epitope. Other than the human 

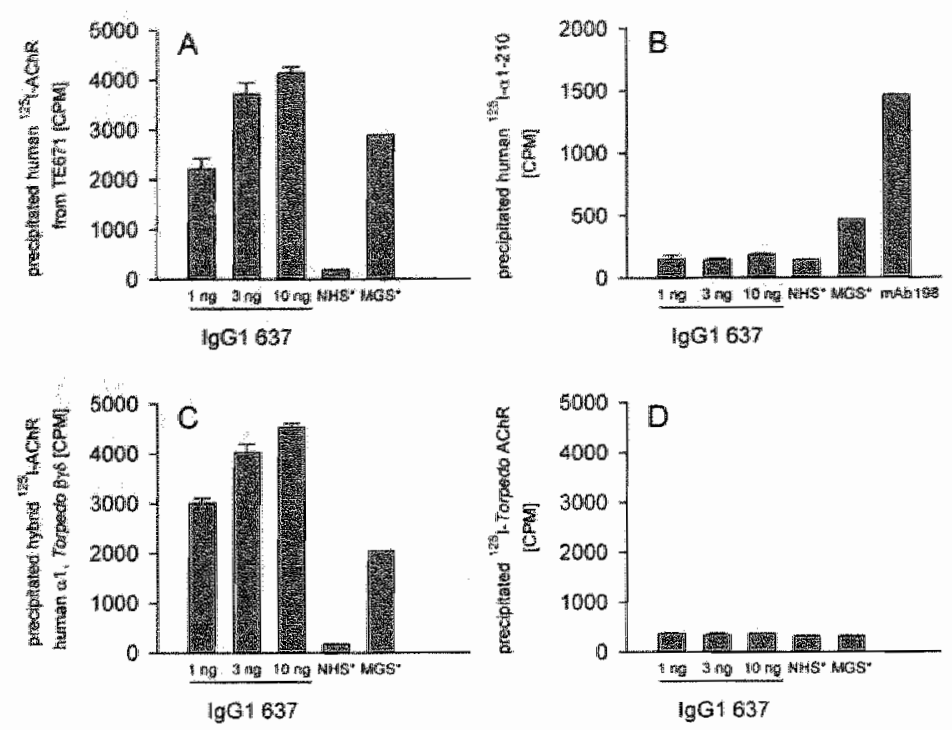

Figure 1. Specificities of the human anti-AChR mAb ligG1637 as determined by radioimmunoassay. (A) IgG1 637 binds to the human AChR from membrane extracts from TE671 cells. (B) There was no binding to the recombinant peptide a $1-210$, representing the extracellular part of the alpha subunit of the human $A C h R$. (C) IgG1637 binds to a recombinant hybrid AChR from membrane extracts of cells expressing the human a1 and the Torpedo $\beta y \delta$ subunits. (D) lgG1 637 does not bind to the wildtype Torpedo AChR. Alsa control MG sera did not crossreact with Torpedo AChR. "NHS - normal human serum; *MGS - serum from myasthenia gravis patients.

AChR, IgG1 637 bound to AChR of rhesus monkeys (Figure 8C), but not of common marmoset monkeys, as found by immunohistochemical staining of primate muscles (Table I).

FACS analysis was used to study antibody binding to intact AChR of cells. The results confirmed that $\operatorname{IgG} \Perp 637$ bound to surface expressed (Figure $2 \mathrm{~A}, \mathrm{~B}$ ) and intracellular fetal AChR of the rhabdomyosarcoma cell line TE671 cells (Figure 2C). Moreover, we tested antibody binding to the thymic myoid cell line MITC (Wakkach et al., 1999) (Figure 2D), because myoid cells express AChR and are possibly involved in the pathogenesis of MG (Shiono et al., 2003). The FACS data suggest that IgG1 637 indeed binds to thymic AChR which supports the hypothesis that the AChR of myoid cells is an autoantigen in $\mathrm{MG}$.

\section{Recombinant $\lg G 1$ and $\lg \mathrm{G} 4$ anti-AChR autoantibodies have the same kinetic properties (apparent affinity and cross-linking) but differ in complement activation.}

The affinities of IgGl 637 and IgG4 637 to human muscle AChR from TE671 cells were compared by a radioimmunoassay (Figure $3 \mathrm{~A}$ ). The results indicate that both antibodies bind the human AChR with the same apparent dissociation constant of approximately $3.5 \mathrm{nM}$. 

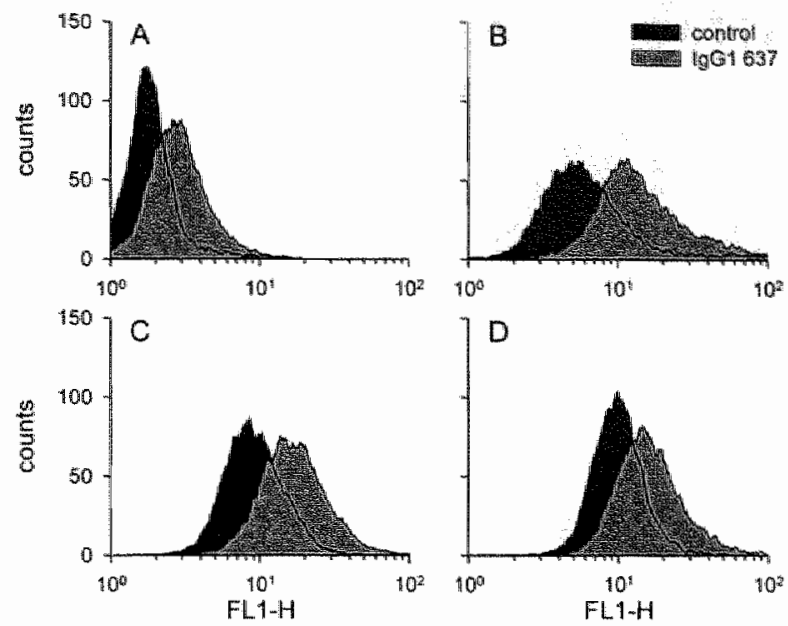

Figure 2. FACS analysis of IgG1 637. The human fetal muscle AChR expressing cell line TE671 was incubated with $\operatorname{lgG}$ 1 637. Shown are (A) the FL-1 intensities of subsequent staining with goat anti-human lg FiTC or (B) goat anti-human lg FitC followed by signal amplification using the EAS Kit. (C) shows staining of permeabilised TE671 cells. (D) The human fetal and adult muscle AChR-expressing cell line MITC was incubated with ug 1637 and stained with goat anti-human Ig FITC. Specific staining is shown in grey, black indicates the background staining.
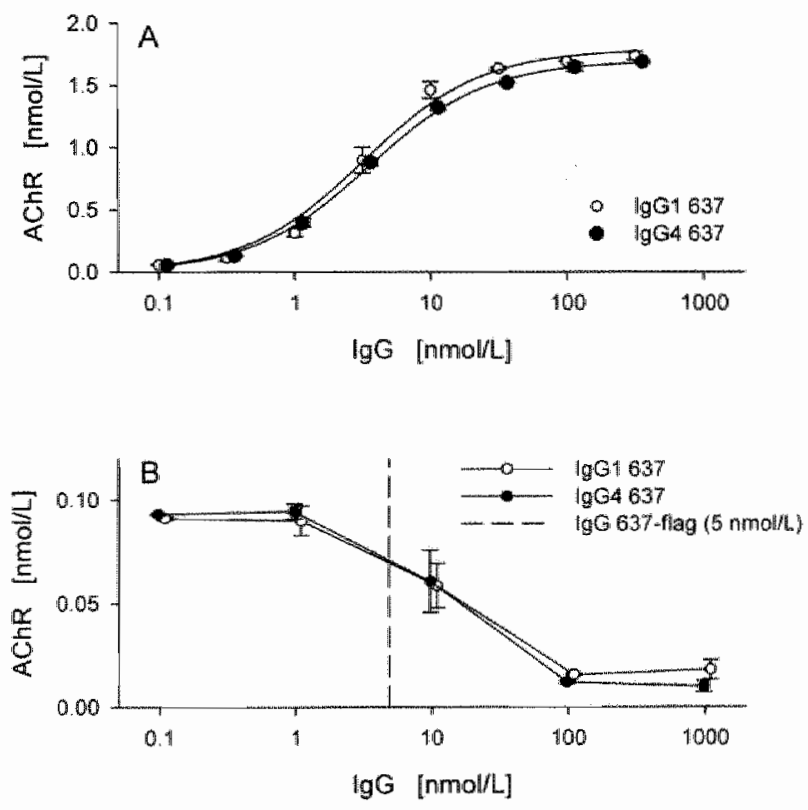

Figure 3. Binding of ligG 637 and $\operatorname{lgG} 4637$ to AChR from TE671 cells measured by radioimmunoassay. (A) Binding curves using dilutions of antibody and a fxed amount of AChR. Data were fitted to a rectangullar hyperbolla. The apparent $\mathrm{Kd}$ is $3.5 \mathrm{nmol} / \mathrm{L}$ for both antibadies. (B) competition of $\mathrm{gg} G 1637 \mathrm{with} \lg \mathrm{G} 4637$. Fixed amounts of AChR and $\operatorname{gG} 1637$ flag were incubated with competitor antibody. Flag tagged antibodies were specifically precipitated with mouse anti flag $\mathrm{mAb} M 2$. The binding of $\mathrm{lgG} 1637$ flag was reduced equally by lgG 637 and $\operatorname{lgG} 4637$, if the concentrations exceeded their $\mathrm{Kd}$. 
The ability of IgG4637 to compete with IgG1 637 was tested in an immunoprecipitation assay using IgGI 637 flag (Figure 3B). For this purpose different concentrations of IgG1 637 or IgG4 637 were pre-mixed with a fixed concentration of IgG1637 flag, which was then incubated with human AChR labelled with ${ }^{25}$ I-a-bungarotoxin. The IgG1 637 and IgG4 637 showed similar inhibition curves thus confirming that the affinities of both isotypes were similar.

The activation of complement by IgG1 637 and IgG4 637 was tested by ELISA (Figure 4). The purified IgG1 637 bound $\mathrm{Clq}$ and activated $\mathrm{C} 3$ and $\mathrm{C} 4$ as efficient as polyclonal human IgG1, whereas the IgG4 637 and the polyclonal IgG4 control did not activate complement via the classical pathway, as expected. Thus, the human IgG1 637 and IgG4637 were suitable tools for studying the role of complement in $M G$.
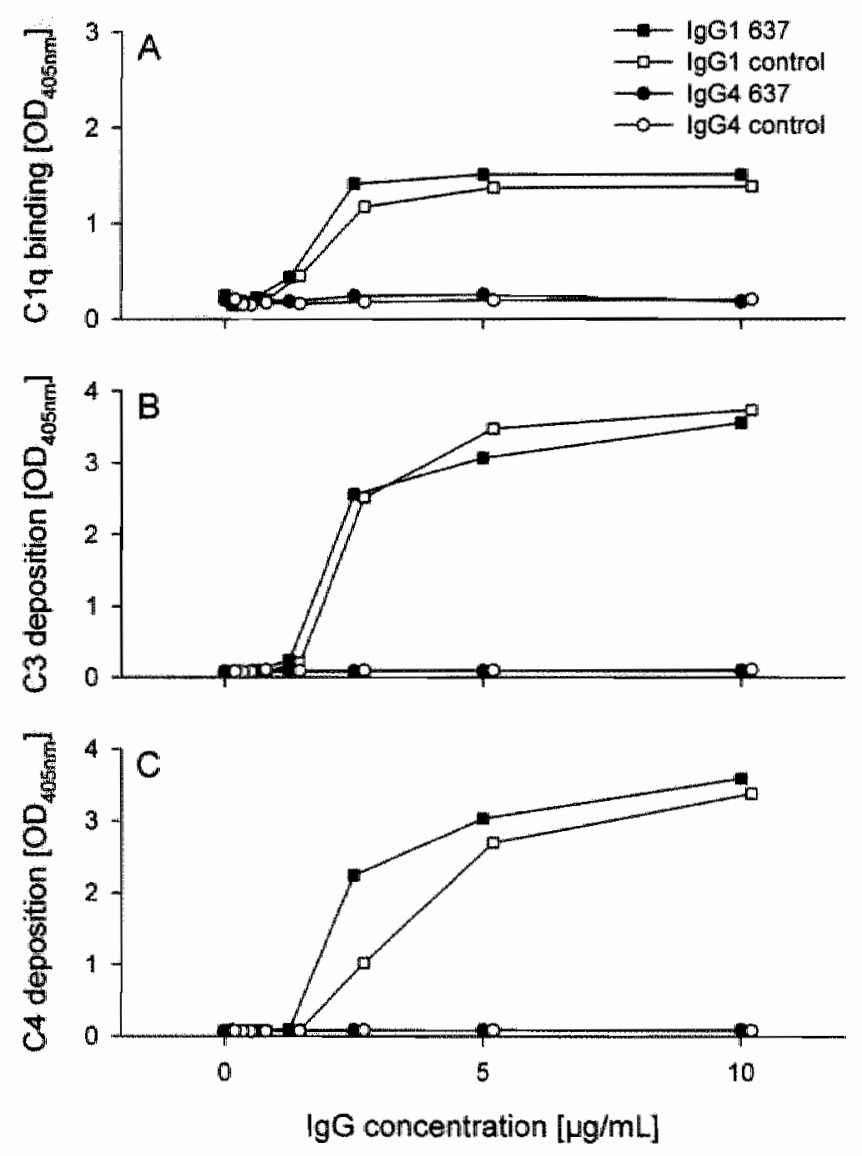

Figure 4. Clq binding and complement activation of IgG1 637 and IgG4 637. The complement activation was determined by ELISA at different stages. (A) Clq binding. (B) C3b deposition and (C) C4b deposition. Polyclonal human lgGi and lgG4 were used as controls (open symbols). 


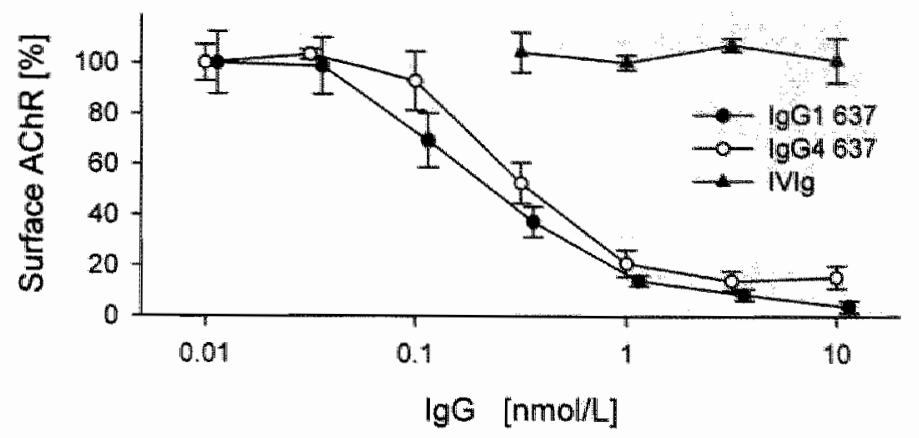

Figure 5. Antigenic modulation of surface AChR of TE671 cells. Confluent cells were incubated with cyclo. heximide and antibodies. IgG 637 and $\lg G 4637$ equally reduced surface $A C h R$ levels, while human immunoglobulin (IVIg) had no effect compared to medium without antibodies.

Because cross-linking anti-AChR antibodies can cause $A C h R$ degradation in cell cultures by antigenic modulation even in the absence of complement, we tested the effect of IgG1 637 and IgG4 637 on cultured TE671 cells. Both antibodies effectively reduced the cell surface AChR levels (Figure 5). Control immunoglobulin from human polyclonal IVIg preparations had no effect on the AChR levels. The result indicated that both $\operatorname{IgG} 637$ antibodies caused antigenic modulation by cross-linking the AChR.

\section{Recombinant human IgG1 anti-AChR autoantibodies induce MG in rhesus monkeys.}

In order to test the ability of IgG4637 to compete with IgG1 637 in vivo, first an animal model had to be established. Since IgG1 637 only binds to the human and macaque AChR (Table I), a passive transfer experiment with IgG1 637 in rhesus monkeys (Macaca mulatta) was performed. First, the binding of IgG1 637 to endplates in human and mon-

Table II. Hin vivo competition of $\|$ gG1 637 with $\| \mathrm{gG} 4637$

\begin{tabular}{|c|c|c|c|c|}
\hline number of animals & antibady & 3 doses of & $\begin{array}{l}\text { decremerital fresponse upon } \\
\text { repetitithe nerve stirnulation }\end{array}$ & dinical syusptoms \\
\hline 1 & $\lg 61637$ & $0.5 \mathrm{mg} / \mathrm{kg}$ & - & - \\
\hline$\pi$ & $\lg 61637$ & $1.0 \mathrm{mg} / \mathrm{kg}$ & + & - \\
\hline 1 & $\lg 61637$ & $5.0 \mathrm{mg} / \mathrm{kg}$ & + & + \\
\hline 4 & $\begin{array}{c}\operatorname{lWlg} \\
\lg 61637\end{array}$ & $\begin{array}{l}5.0 \mathrm{mg} / \mathrm{kg} \\
1.7 \mathrm{mg} / \mathrm{kg}\end{array}$ & $+(4 / 4)$ & $+(3 / 4)$ \\
\hline 2 & $\begin{array}{l}\lg 64637 \\
\operatorname{lWlg}\end{array}$ & $\begin{array}{l}5.0 \mathrm{mg} / \mathrm{kg} \\
1.7 \mathrm{mg} / \mathrm{kg}\end{array}$ & $-(2 / 2)$ & $=(2 / 2)$ \\
\hline 5 & $\begin{array}{l}\lg 64637 \\
\lg 61637\end{array}$ & $\begin{array}{l}5.0 \mathrm{mg} / \mathrm{kg} \\
1.7 \mathrm{mg} / \mathrm{kg}\end{array}$ & $-(5 / 5)$ & $\cdot(5 / 5)$ \\
\hline
\end{tabular}


key biopsies was analyzed by immunohistochemisty. By qualitative comparison of staining interisities, it was calculated that doses of $1.5 \mathrm{mg} / \mathrm{kg}$ or $3 \mathrm{mg} / \mathrm{kg}$ could be used safely and these doses were injected in one monkey for each concentration. Acetylcholine esterase treatment was ready to be injected in case animals developed a myasthenic crisis with respiratory problems. However, none of the treated monkeys showed clinical symptoms of MG (Table II). Seven days after the first injection, the animals were anesthetized and the neuromuscular transmission was tested by measuring the CMAP during RNS. The animal injected with $1.5 \mathrm{mg} / \mathrm{kg}$ showed no decrement, but the monkey injected with $3 \mathrm{mg} / \mathrm{kg}$ had a decremental response when tested on day 7 (data not shown), demonstrating disturbed neuromuscular transmission. Subsequently, higher doses of IgG1 637 were tested. At $5 \mathrm{mg} /$ $\mathrm{kg}$ and $15 \mathrm{mg} / \mathrm{kg}$ IgG1 637 caused clinical symptoms in all animals. Symptoms started between 1 and 5 days after the first injection and lasted up to 7 days. At the peak of the disease, animals were hypo-active, could not climb because of weakness of the limbs, hands and feet, and had difficulty eating, but no respiratory problems. This phase lasted for $2-3$ days. Three out of four animals injected with $5 \mathrm{mg} / \mathrm{kg}$ and the animal injected with $15 \mathrm{mg} / \mathrm{kg}$ IgG1 637 had a decremental response when tested 7 days after the first injection, even if their condition had already begun to improve. On average, the clinical symptoms paral. leled the antibody titer in the serum of the animals, which declined slowly after the third injection of $\operatorname{lgG} 1637$ (Figure 6).
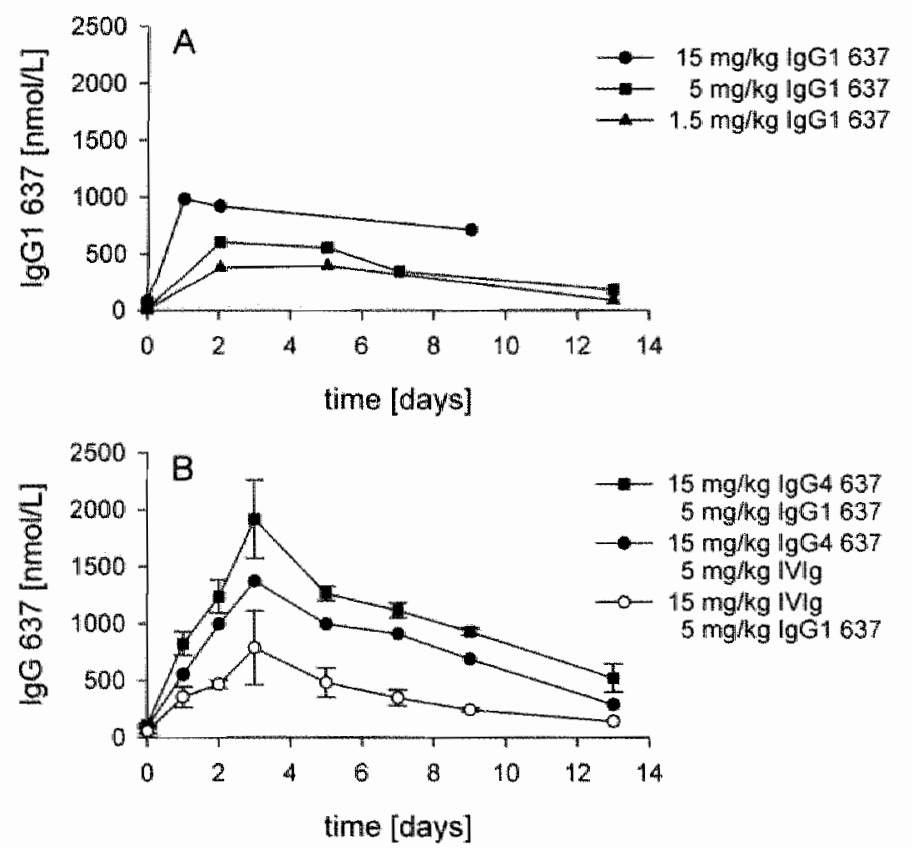

Figure 6. Anti-AChR antibody titer in the serum of thesus monkeys. (A) Anti-AChR antibody titer in moinkeys injected with different doses of $\mathrm{Ig} G 1637$ on day 0,1 and 2. (B) Average anti-AChR antibody titer ( \pm SD) in monkeys injected on day 0,1 , and 2 with $\lg G 1637(n=4), \lg G 4637(n=2)$ and $\lg G 1637+\lg G 4637+1 \mathrm{~W} / \mathrm{g}(\mathrm{n}=5)$. Additional injections of 1 ilg were given to obtain an equal total dose of human antibody. 


\section{Recombinant human lgG4 anti-AChR autoantibodies prevent MG in rhesus monkeys.}

The protective effect of $\operatorname{IgG} 4637$ was tested in vivo with experimentally nave monkeys: Animals received a total of 6 injections on 3 experimental days: In the morning of each day, animals were injected with $5 \mathrm{mg} / \mathrm{kg} /$ day $\mathrm{IgG} 4$ or control antibody (human IVIg) and six hours later they were injected with $1.7 \mathrm{mg} / \mathrm{kg} / \mathrm{day}$ lgGl 637 or control antibody (Table II). The animals $(n=4)$ receiving a total of $5 \mathrm{mg} / \mathrm{kg} \operatorname{IgGI} 637$ were clinically ill and 3 animals showed decrement on RNS at all tested stimulation frequencies (CMAP at $5 \mathrm{~Hz}$ stimulation is shown in Figure 7A). To asses whether the IgG4 637 was also pathogenic, we treated two additional animals with a high dose of this antibody (total dose $15 \mathrm{mg} / \mathrm{kg}$ ). Interestingly, these animals did not show any clinical symptoms or decrement at any stimulation frequency (Figure $7 \mathrm{~B}$ ). Animals $(n=5)$ which received IgG4 637 in the mornings and IgG1 637 six. hours later on the same experimental days, did not develop any clinical symptoms or a decremental response at any stimulation frequency (Figure $7 \mathrm{C}$ ), despite the fact that they had the highest titer of antibodies against the $A C h R$ (Figure $6 B$ ). The treatment effect is significant ( $p<0.01$ for clinical illness and $\mathrm{p}=0.048$ for decrement results; 2 -tailed Fischer's exact test).

The serum-titer of IgG1 637 and $\operatorname{IgG} 4637$ decreased more rapidly than can be expected from the normal IgG half life in humans of approximately $2 \mathbb{1}$ days. The half life of IgG1 637 in this study was approximately 5 days and the half life of combined. IgG 1637 and IgG4 637 was 6 days. This can partly be explained by the absorption of the antibodies at the endplates (Graus et al., 1993), but also by the lower stability of human IgG in macaques (Lin et al., 1999). A decreased half life of anti-AChR antibodies has been observed in neonatal MG after passive transfer from mother to child (Vincent, 1980).

Intercostal biopsies were taken after the EMG measurements from 3 rhesus monkeys; each representing an experimental group (Table II). Unfortunately, the biopsies of the animals treated with either IgG1 or IgG4 did not contain endplates. The intercostal biopsy taken from an animal treated with both $\operatorname{IgG1} 637$ and $\operatorname{IgG} 4637$ contained endplates that stained with mouse antibodies directed against respectively human IgGl and human IgG4. Hence, both IgGI 637 and $\operatorname{IgG} 4637$ were located at the neuromuscular junctions (Figure $8 \mathrm{C}, \mathrm{D}$ ). The endplates double stained intensively for both $\mathrm{ACh}$ (using abungarotoxin, Figure 8B, left) and membrane attack complex (MAC, C5b-9) (Figure 8B, middle). The same result was obtained using a staining for the complement protein $\mathrm{C} 4$ (not shown). No activated complement proteins were found on endplates of an untreated control animal (Figure 8A). Thus, IgG4 637 bound to the rhesus monkey endplates but did not completely prevent IgG1 637 binding and complement activation. However, electron microscopic analysis of the biopsies showed that the ultrastructure of postsynaptic membrane was preserved (Figure $8 \mathrm{E}$ ). The typical myasthenic destruction of the postsynaptic folding was absent, indicating that the pastsynaptic membrane tolerated some degree of complement activation. 

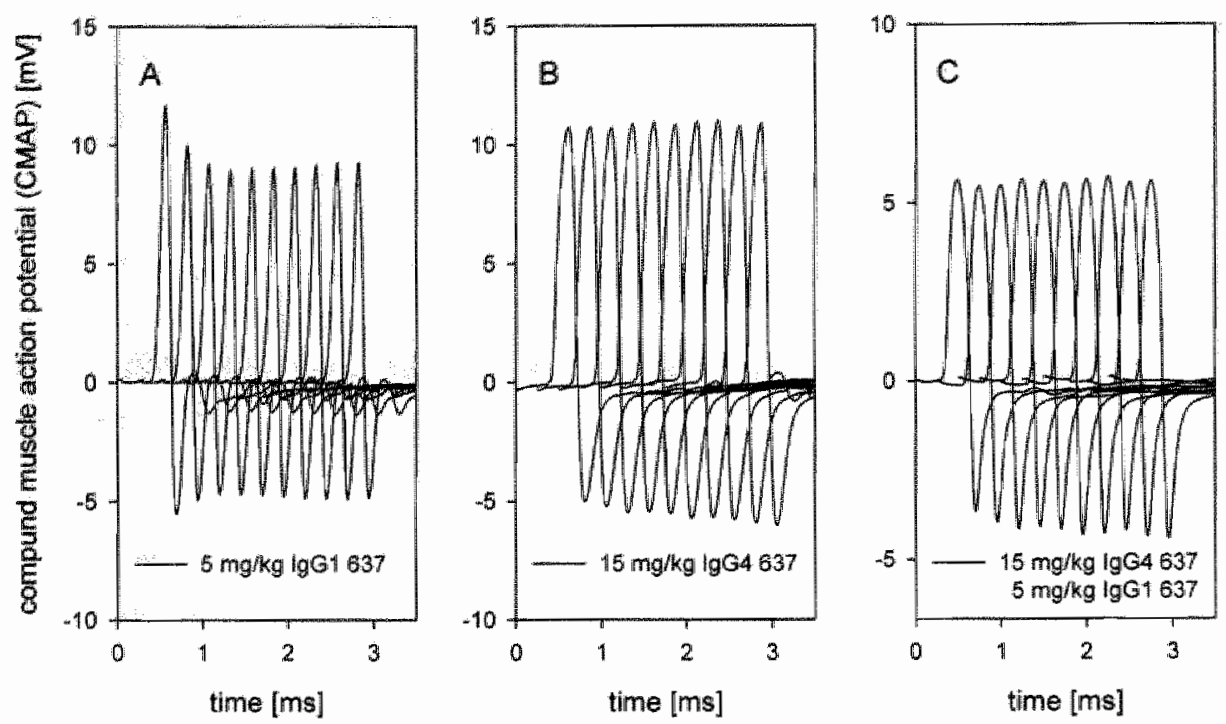

Figure 7. Compound muscle action potentials (CMAP) of the extensor digitorum brevis muscle during RNS of the peroneal nerven (A) Decremental response of the CMAP in an animal that received $5 \mathrm{mg} / \mathrm{kg} /$ day $/ \mathrm{lgG} 1637$. Both the area and the ampllitude of the negative (upward) peak decreased more than $22 \%$ at $5 \mathrm{~Hz}$. (B) There was no decremental response of the CMAP in an animal that recelved $15 \mathrm{mg} / \mathrm{kg} \mathrm{gGG}$ 637. (C) There was no decremental response of the CMAP in an animal that received $15 \mathrm{mg} / \mathrm{kg} /$ day $\mathrm{lgG} 4637$ and $5 \mathrm{mg} / \mathrm{kg} / \mathrm{day}$ lgG1 637.

Table III. Numerical data of the repetitive nerve stimulation tests shown in Figure 7

\begin{tabular}{|c|c|c|c|c|c|c|c|c|c|c|c|c|}
\hline & & $1.7 \mathrm{mg} / \mathrm{kg} /$ & ay $\lg 6163$ & & & $0 \mathrm{mg} / \mathrm{kg} / \mathrm{d}$ & ay $\lg 6463$ & & & $5.0 \mathrm{mg} / \mathrm{kg} / \mathrm{h}$ & $\begin{array}{l}\text { ay } \lg 5463 \\
\text { day } \lg 616\end{array}$ & \\
\hline & & mulation a & $5 . \mathrm{Hz}_{2}, 60.5$ & & & nulation at & $5 \mathrm{~Hz}_{2}, 53.1$ & & & mullation at & $5 \mathrm{~Hz}_{t} 62.9$ & \\
\hline $\begin{array}{l}\text { Pot: } \\
\text { No. }\end{array}$ & $\begin{array}{c}\text { Peak Amp. } \\
{[\mathrm{nnV}]}\end{array}$ & 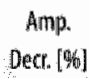 & $\begin{array}{c}\text { Ared } \\
\text { [mWms] }\end{array}$ & $\begin{array}{c}\text { Area Decr. } \\
\qquad \%]\end{array}$ & $\begin{array}{l}\text { Peak Amp. } \\
\text { [mV] }\end{array}$ & 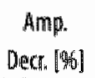 & $\begin{array}{c}\text { Area } \\
\text { [mVims]] }\end{array}$ & $\begin{array}{c}\text { Area Decr. } \\
{[\%]}\end{array}$ & $\begin{array}{l}\text { Peak Amplo. } \\
\text { [mV] }\end{array}$ & $\begin{array}{c}\text { Amp. } \\
\text { Decr. }[\%] \text {. }\end{array}$ & $\begin{array}{l}\text { Area } \\
\text { [mWms] }\end{array}$ & 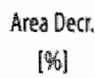 \\
\hline 1 & $11: 62$ & $a$ & 13.30 & 0 & 10.80 & 0 & 21.40 & 0 & 5.64 & 0 & 11.70 & 0 \\
\hline 2 & 9.96 & 14 & 11.20 & 16 & 10,88 & -1 & 20.90 & 2 & 5.58 & 1 & 11.10 & 5 \\
\hline 3 & $9: 17$ & 21 & 10.20 & 23 & 10.87 & -1 & 20.60 & 4 & 5.52 & 2 & 11.00 & 6 \\
\hline 4 & 8.91 & 23 & 9.97 & 25 & 10.89 & -1 & 21.00 & 2 & 5.65 & 0 & 11,40 & 3 \\
\hline 5 & 9.03 & 22 & 9.95 & 25 & 10.97 & -2 & 21.00 & 2 & 5.72 & -1 & 111.20 & 4 \\
\hline 6 & 9.02 & 22 & 9.93 & 25 & 10.95 & -1 & 20.70 & 3 & 5.61 & 1 & 11.00 & 6 \\
\hline 7 & 9.07 & 22 & 9.91 & 25 & 10.89 & -1 & 21.10 & 1 & 5.61 & 1 & 11.30 & 3 \\
\hline 8 & 9.15 & 21 & 9.97 & 25 & 11.02 & -2 & 21.00 & 2 & 5.73 & -2 & 11.40 & 3 \\
\hline 9 & 9.21 & 21 & 10,000 & 25 & 10.97 & -2 & 20.44 & 5 & 5.69 & -1 & 11.00 & 6 \\
\hline 10 & 9.28 & 20 & 10.00 & 25 & 11.04 & -2 & 20.30 & 5 & 5.60 & 1 & 11.00 & 6 \\
\hline
\end{tabular}


AChR

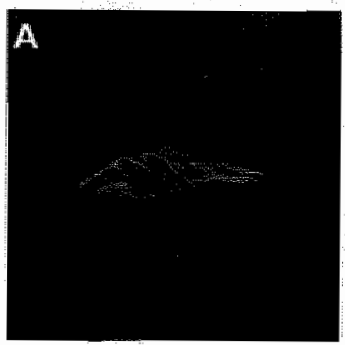

B

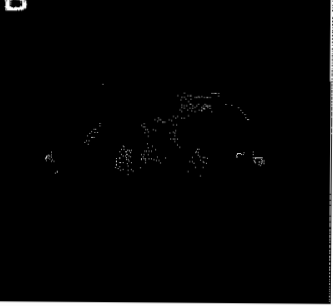

MAC
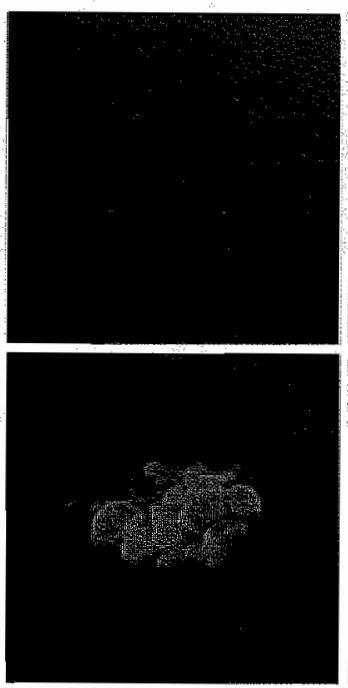

\section{merge}

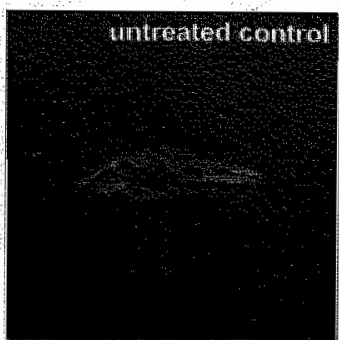

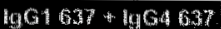

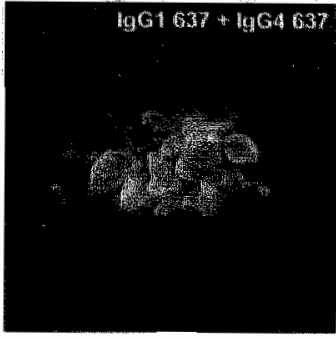

IgG4
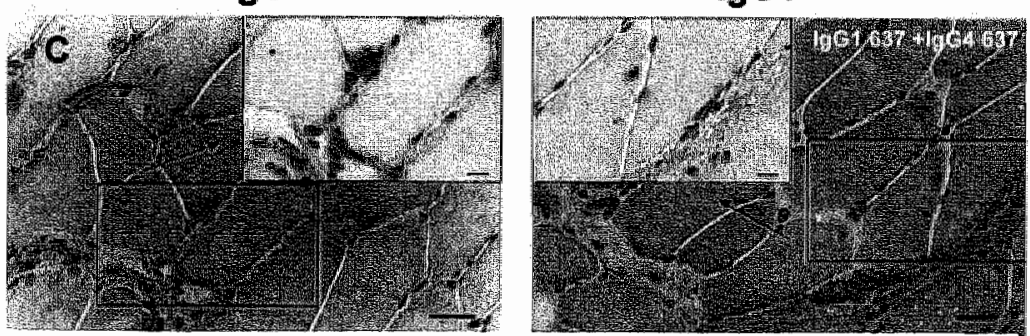

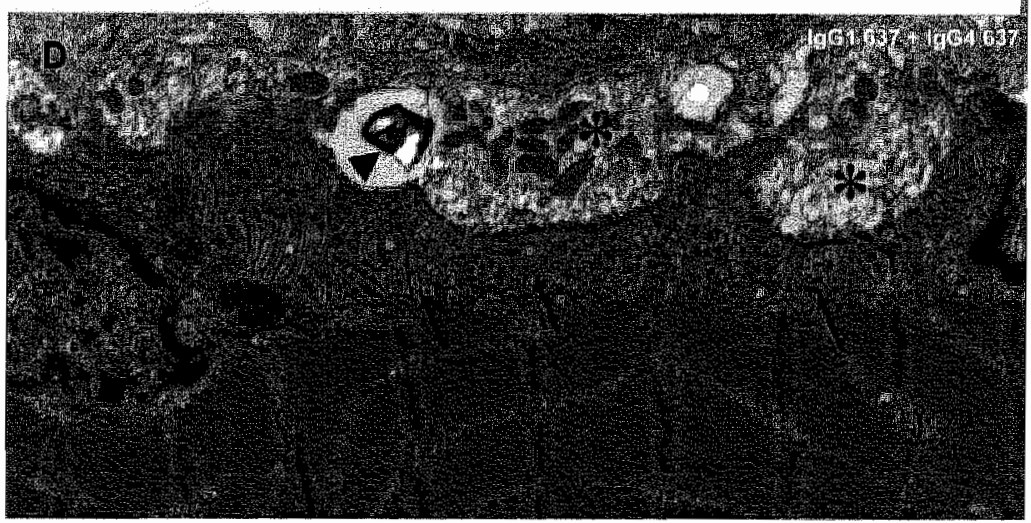

Figure 8. Analysis of endplates in an intercostals biopsy from (A) an untreated control mesus monkey and (B. D) from a rhesus monkey 7 days after the first injection of $5 \mathrm{mg} / \mathrm{kg} / \mathrm{day} / \mathrm{lgG} 4637$ and $1.7 \mathrm{mg} / \mathrm{kg} / \mathrm{day} / \mathrm{gG} 1637$. (A, B) Cryosections were double stained for the AChR using rhodamime labeled a-bungarotoxin and for the membrane attack complex of complement using mAb aE11. (C) Staining of human $\| \mathrm{gG}$ and lgG4. Both antibodles are present at the neuromuscular junctions (arrows) as detected with mouse-anti-human IgG1 (HP6 184) and mouse-anti-human IgG4 (MH164-4) respectively. Nuclei were stained with hematoxylin (blue). (D) electron micrograph of an endplate region. Arrows indicate some of the intact folds postsynaptic memu* brane, the asterisk shows the nerve terminal and the arrowhead indicates a preparatory artifact. 


\section{Discussion}

In this study we produced human IgGI and IgG4 anti-human AChR auto-antibodies (IgG1 637 and $\operatorname{lgG4637)~and~characterized~them~in~vitro~and~in~a~rhesus~monkey~model.~}$ Our principal finding was that IgG4 can prevent the pathogenic effect of IgGI in a passive transfer MG model. The ability of IgG4 637 to block IgG1 637 binding to the AChR in vivo was confirmed in a competition experiment with a $3: 1$ excess of $\operatorname{IgG} 4637$ : when 5 monkeys were injected with a high dose of $\mathrm{lgG} 4637(15 \mathrm{mg} / \mathrm{kg})$ prior to a pathogenic dose of $\operatorname{lgGI} 637(5 \mathrm{mg} / \mathrm{kg})$ no disease symptoms were observed. Both antibodies bound to the NMI, but intensive staining of AChR was found, in spite of the presence of activated complement. Similarly, an $3: 1$ excess of protective $F\left(a b^{3}\right)_{2}$ fragments competing with pathogenic IgG was also effective in preventing disease symptoms in a passive transfer EAMG model in rats (Richman et al., 1998),

Both antibodies bind to the a-subunit of the human muscle-AChR. In a previous report it has been shown that Fab 637 competes with monoclonal antibodies and patient sera for binding to the MIR (Graus et al., 1997) without interfering with the ion channel function. Compared to Fab fragments, full size antibodies have a higher avidity because of their bivalent binding. Moreover, there is a higher chance of steric hindrance of autoantibody binding to nearby binding sites on the AChR that would not be affected by Fab fragments. Indeed, it has been suggested that anti-MIR antibodies can also prevent binding of antibodies to other AChR epitopes (Lennon and Griesmann, 1989).

The apparent dissociation constant of both IgGI 637 and IgG4 637 for human AChR was determined to be $3.5 \mathrm{nmol} / \mathrm{L}$ as measured by RIA using AChR-rich cell membrane extracts. Fab 637 was isolated from a patient with an anti AChR-antibody titer of $24 \mathrm{nmol} / \mathrm{L}$ (Graus et al., 1997). Most MG patients with anti-AChR antibodies have titers higher than. $5 \mathrm{nmol} / \mathrm{L}$ (Rodgaard et al., 1987). Based on an average total amount of $20 \mathrm{nmol}$ of AChR (Vincent, 1980) and $10 \mathrm{~L}$ extracellular fluid, IgG 637 could potentially bind to a large proportion of muscle AChR at physiologic concentrations in MG patients.

IgG1 637 and IgG4 637 caused degradation of surface AChR in TE671 cells to the same extend. This suggested that both antibodies were capable of crosslinking the AChR. Approximately $50 \%$ of the surface $A C h R$ was degraded by antigenic modulation at a concentration of $0.32 \mathrm{nM} \operatorname{lgGl} 637$ or $\operatorname{lgG} 4637$. Therefore, antigenic modulation cannot explain the differences in pathogenicity of the antibodies.

As expected from previous work, $\operatorname{IgG} 4637$ did not bind and activate complement. The ability to compete with IgG1 637 was confirmed by a radioimmunoassay using IgG1 637 Alag (Figure $3 B$ ). In order to test the competition and the potential protection of AChR in vivo, the crossreactivity of IgGl 637 with the AChR of other species was tested. which suggested that these experiments could only be performed in macaques. One advantage of using a non-human primate model is the similarity to the human immune system. In this respect it is interesting to note that the presence of all four IgG subclasses has been demonstrated at the genomic and RNA level with around $90 \%$ homology to their human counterparts (Scinicariello et al., 2004), while 3 subclasses have been identified at 
the protein level in rhesus macaques (Calvas et al, 1999; Martin, 1982; Shearer et al., 1999). Little is known about effector functions of the macaque antibodies, but has been shown that the amino acids essential for complement binding in human IgG are preserved in all macaque IgG subclasses (Calvas et al., 1999; Scinicariello et al., 2004). Our results suggest that IgG4 637 binds to endplates of rhesus monkeys in vivo, but does not impair neuro. muscular transmission when injected at a dose of $15 \mathrm{mg} / \mathrm{kg}$. Conversely, IgG1 637 impairs neuromuscular transmission at a dose of $3 \mathrm{mg} / \mathrm{kg}$ and causes muscle weakness at a dose of $5 \mathrm{mg} / \mathrm{kg}$ or $15 \mathrm{mg} / \mathrm{kg}$. This suggests that the function of the human $\mathbb{I g G}$ subclasses $\mathbb{1}$ and 4 with respect to complement fixation are maintained in the rhesus monkey.

Taken together the results rebut the possibility that IgG4 anti MIR auto-antibodies aggravate $\mathrm{MG}$, but support the hypothesis that IgG4 can reduce immune mediated reactions caused by IgGl of the same antigen specificity in chronic diseases. Moreover they support the complement hypothesis of MG (Richman et al., 1998). The concern was not substantiated in this passive transfer MG study that recombinantly produced IgG4 [which is bivalent as opposed to its in viwo counterpart (Aalberse et al., 1999; van der Zee et al., 1986)] could be pathogenic due to crosslinking and antigenic modulation of the $A C h R$ (see Figure 5). This also suggests that antigenic modulation is probably not the primary pathogenic mechanism of IgG1 637. Antigenic modulation has also been shown to be insufficient to cause muscle weakness in passive transfer studies of antibodies from EAMG with mice and rats (Berman and Heinemann, 1984; Lennon et al., 1978), but it is noteworthy that in contrast to humans and non-human primates the safety margin of neuromuscular transmission is considerably larger in rodents and thus overt weakness is much less likely to occur. Further experiments will be necessary to test the efficacy of long term IgG4 immunotherapy in this passive transfer model of MG or in EAMG. Concerns have been raised with respect to effector functions of $\operatorname{IgG4}$, because it mediated antibody-dependent cellular cytotoxicity (ADCC) in some individuals (Greenwood et al., 1993). However, it has been suggested that ADCC is not responsible for the destruction of the NMI in EAMG induced by anti-AChR antibodies, firstly, because depletion of mononuclear cells does not prevent the disease (Hoedemaekers et al, 1997) and secondly, because anti-AChR receptor antibodies are not pathogenic in the absence of complement (Lennon et al., 1978; Tuzun et al., 2003). Moreover, mononuclear cells are not always observed at the NMJ in muscle biopsies from anti-AChR antibody-positive MG patients (Engel et al., 1976; Maselli et al., 1991).

Future studies might investigate three potential mechanisms by which $\operatorname{IgG} 4637$ could reduce the immune-response against the $A C h R$ in $M G$ patients. Initially, it could block binding of pathogenic IgGl anti-AChR autoantibodies at the NMI and reduce complement-mediated damage, as we have shown in this study. Moreover, it has been proposed that immune complexes containing breakdown products of the postsynaptic membrane

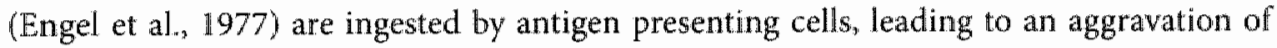
EAMG (Tuzun et al., 2004). This in turn may stimulate the APCs to attract other inflammatory (mononuclear) cells and to stimulate $\mathrm{T}$ cells. IgG subclasses display substantial differences in their capacity for lymphocyte activation and this strongly depends on FC 
receptor binding on phagocytic cells (Nimmerjahn and Ravetch 2005 ). A long term treatment with $\operatorname{lgG} 4637$ could lead to a reduced uptake of immune complexes by macrophages and B-cells; because IgG4 interacts only weakly with Fc receptors. Finally, IgG4 637 could possibly also protect $\mathrm{AChR}$-expressing thymic cells from which the myoid cell line MITC was derived (Figure 2 ). The subsequent reduction in antigen presentation might exert an immuno-modulatory effect.

In another human autoimmune disease, polyradiculoneuritis of the Guillain-Barre type, the neuromuscular blocking action of intrinsic antibodies to gangliosides can be inhibited by polyclonal IgG as used in commercially available therapeutic IgG preparations. It has been speculated that in this condition naturally occurring low-affinity and low-titer antibodies to glycoconjugates as contained in the normal donor derived IgG preparations could sterically hinder the blocking antibodies (Buchwald et al., 2002). The present work is a major advance in proving that this type of mechanism does exist, at least in MG. The concept of blocking IgG4 auto-antibodies in MG could be helpful for the understanding of other antibody mediated autoimmune diseases or allergies (type I-III hypersensitivities), which could possibly lead to targeted immunotherapies.

\section{Acknowledgements}

We are very grateful to $D r$. M. Stassen for the fruitful discussions about protective recombinant antibodies and to Dr. P. Kreczmanski, Dr. Y. Temel and Dr. G. Brascamp for taking the intercostal biopsies. We would also like to thank M. van de Waarenburg, Dr. B. Machiels. and L. van Geest for their excellent technical assistance. This work was supported by grants from the Prinses Beatrix Fonds, LAssociation Française contre les Myopathies, a Marie Curie Fellowship and the project grant "QLG3-CT-2001-00225" of the European Community program "quality of life and management of living resources". Conflicting financial interests: This study has been financially supported by Genmab BV, Utrecht, The Netherlands. The results of this study have been filed in a patent application.

\section{References}

Aalberse RC, Schuurnan I. IgG4 breaking the rules. Immunology $2002 ; 105 ; 9-19$.

Aalberse RC, Schuturman J, van Ree R. The apparent monovalency of human IgG4 is due to bispecificity. Int Arch Allergy Immurwol 1999; 118:187 9 .

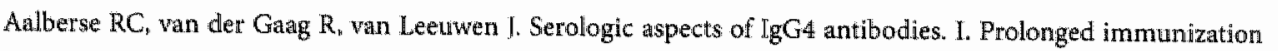
results in an IgG4-restricted response. J Immunol 1983; 130: 722-6.

Almon RR, Andrew CG, Appel SH. Serum globulin in myasthenia gravis: inhibition of alpha-bungarotoxin binding to acetylcholine receptors. Science $1974 ; 186: 55-7$.

Berman PW, Heinemann SF. Antigenic modulation of junctional acetylcholine receptor is not sufficient to account for the development of myasthenia gravis in receptor immunized mice. I Immunol 1984; 132: 711-7.

Buchwald B, Ahangari R, Weishaupt A, Toyka KV. Intravenous immunoglobulins neutralize blocking antibodies in Guillain-Barre syndrome. Ann Neurol 2002; 51: 673-80. 
Bufler J, Kahlert S, Tzartos S, Toyka KV, Maelicke A, Franke C. Actwation and blockade of mouse musde nicotinic channels by antibodies directed against the binding site of the acetylcholine receptor. I Physiol $1996 ; 492$ $(\mathbb{P}+1): 107-14$.

Calvas P, Apoil P, Fortenfant $F$, Roubinet $\mathbb{F}$, Andris I, Capra $D$, et al Characterization of the three mmunoglobulin G subclasses of macaques. Scand I Imminol 1999; 49:595-610.

Cragg MS, French RR, Glennie MJ. Signaling antibodies in cancer therapy. Curr Opin Inmuinol 1999:11:541-7.

Engel $\mathrm{AG}_{0}$ Lambert $\mathrm{EH}$, Howard FM. Inmune complexes (IgG and C3) at the motor end-plate in myasthenia gravis: ultrastructural and light microscopic localization and electrophysiologic correlations: Mayo Clin Proc 1977; $52: 267-80$.

Engel $A G$, Tsujihata $M$, Lambert $E H$, Lindstrom JM, Lennon. WA. Experimental autoimmune myigsthenia gravis: a sequential and quantitative study of the neuromuscular junction ultrastructure and alectrophysiologic correlations. J Neuropathol Exp Neirol 1976; 35:569-87.

Farran I, Portolano S, Willcox N, Vincent A, Jacobson L, Newsom-Davis J, et al. Diverse Fab specific for acetylcholine receptor epitopes from a myasthenia gravis thymus combinatorial library. Int Immunol 1997; 9: $1311-8$.

Fertuck HC, Salpeter MM. Quantitation of junctional and extrajunctional acetylcholine receptors by electron microscope autoradiography after ${ }^{25: 5-a l p h a-b u n g a r o t o x i n ~ b i n d i n g ~ a t ~ m o u s e ~ n e u r o m u s c u l a r ~ j u n c t i o n s . ~] ~}$ Cell Biol 1976; 69: 144-58.

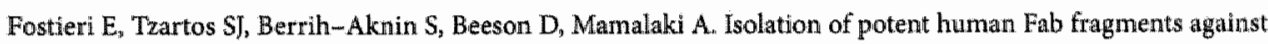
a novel highly immunogenic region on human muscle acetylcholine receptor which protect the receptor from myasthenic autoantibodies. Eur J Immunol 2005; 35:632-43.

Garraud $O$, Perraut R, Riveau G, Nutman TB. Class and subclass selection in parasite-specific antibody responses. Trends Parasitol 2003; 19:300-4.

Gomez $C M$, Richman DP. Anti-acetylcholine receptor antibodies directed against the alpha-bungarotoxin binding site induce a unique form of experimental myasthenia. Proc Natl Acad Sci U S A 1983; 80: 4089 93.

Graus YF, de Baets MH, Parren PW, Berrih-Aknin S, Wokke J, van Breda Vriesman PI, et al. Human anti-nicotinic acetylcholine receptor recombinant Fab fragments isolated from thymus-derived phage display libraries from myasthenia gravis patients reflect predominant specificities in serum and block the action of pathogenic serum antibodies. IImmunol 1997; 158: 1919-29.

Graus YM, Verschuuren J), Spaans F, Jennekens F, van Breda Vriesman PJ, De Baets MH. Age-melated resistance to experimental autoimmune myasthenia gravis in rats. I Immunol 1993; 150:4093-103.

Greenwood $\mathrm{J}_{\text {: }} \mathrm{Clark} \mathrm{M}$, Waldmann $\mathrm{H}$. Structural motifs involved in human IgG antibody eftector functions. Eur Jimmunol 1993; 23: 1098-104.

Gu $Y_{*}$ Hall $Z$ W. Immunological evidence for a change in subunits of the acetylcholine receptor in developing and denervated rat muscle. Neuron 1988; 1:117-25.

Heinemann $S_{4}$ Bevan $S_{3}$ Kullberg R, Lindstrom J, Rice I. Modulation of acetylcholine recepton by antibody against the receptor. Proc Nat Acad Sci U 5 A 1977; 74: 3090 4.

Hoch W, McConville J, Helms S, Newsom-Dawis J, Melms A, Vincent A. Auto-antibodies to the receptor tyrositne kinase MuSK in patients with myasthenia gravis without acetylcholine teceptor antibodies. Nat Med 2001: 7: 365-8. 


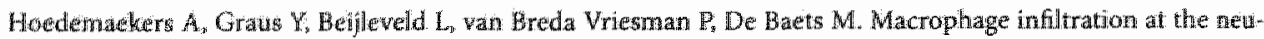
romuscular junction does not contribute to AChR loss and age-related resistance to EAMG. I Neurommanol 1997: $75: 147-55$.

Im $3 \mathrm{H}_{3}$ Harchan D, Mait PK, Raveh L, Souroujon MC. Fuchs S. Suppression of experimental myasthenia grevils, a Be cell-nediated antoimmune disease, by blockate of IL-18. Faseb $12001 ; 15: 2140-8$.

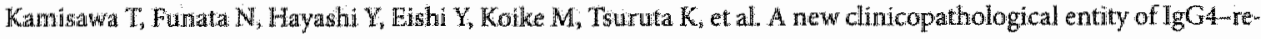
lated autoimmine disease. I Castroenterol 2003; 38: 982-4

Ka0 1, Drachman DB. Myasthenic immunoglobulin accelerates acetylchol ine receptor degradation. Science 197\%; $196.527 \cdots 9$

Lang $B$, Richardson $G$, Rees ), Vincent A, Newsom-Davis J. Plasma from myasthenia grawis patients reduces acetylcholine receptor agonist induced Nat fux into TE671 cell line. J Neuroimmunal 1988; 19: 141-8.

Lemnon $Y A$, Griesmann $G E$. Evidence against acetylcholine receptor having a main immunogenic region as target for autoartibodies in myasthenia gravis. Neurology 1989; 39: 1069-76.

Lennon WA, Seybold ME, Lindstrom JM, Cochrane C, Ulevitch R. Role of complement in the pathogenesis of experimental autoinmune myasthenia gravis. I Exp Med 1978; 147: 973-83.

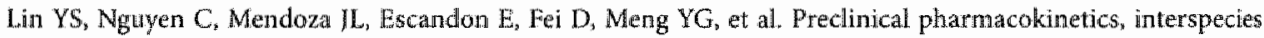
scaling, and tissue distribution of a humanized monoclonal antibody against wascular endothelial growth factor. J Pharmacol Exp Ther 1999; 288; $371-8$.

Lindstrom I, Einarson B, Tzartos S. Production and assay of antibodies to acetylcholine receptors. Methods Erzymol 1981:74 Pl C: 432-60.

Lindstrom IN, Lngel AG, Seybold ME, Lennon VA, Lambert EH " Pathological mechanisms in experimental antoimmune myasthenia gravis. II. Passive transfer of experimental autoimmune myasthenia gravis in rats writh anti-acetylcholine recepotr antibodies. J Exp Med 1976a; 144: 739-53.

Lindstrom JM, Lennon VA, Seyboid ME, Whittingham S. Experimental autoimmune myasthenia gravis and mymsthenia gravis: biochemical and immunochemical aspects. Ann $N$ Y Acad Sci 1976b; 274: 254-74.

Loutrari H, Kokla A, Trakas N, Tzartos S). Expression of human-Torpedo hybrid acetyllcholine receptor (AChR) for analysing the subunit specificity of antibodies in sera from patients with myasthenia gravis (MG). Clin Exp Immunol 1997; 109:538-46.

Loutrari H. Kolda A, Tzartos $\$$. Passive transfer of experimental myasthenia gravis via antigenic modulation of acelyticholine receptor. Eur / Immunol 1992: 22:2449-52.

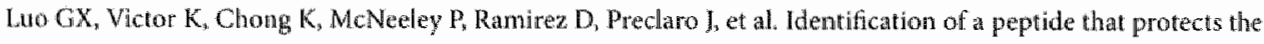
human acetylcholine receptor against antigentic modulation. IInmunol Methods 2001; 251: 177-86.

Luther MA, Schoepfer $\mathrm{R}$, Whiting $\mathrm{P}$, Casey $\mathrm{B}$, Blatt $\mathrm{Y}_{2}$ Montal $\mathrm{M} \mathrm{S}_{3}$ et al. A muscle acetylcholine receptor is ex.pressed in the human cerebellar medulloblastoma cell line "TE671. INeurosci 1989; 9: 1082-96.

Martin LN. Chromatographic fractionation of thesus monkey (Macaca mulatta) IgG sulbclasses using deae cellulose and protein A-sepharose. I Immunol Methods 1982; 50; 319-29.

Maselli RA, Richman DP, Wollmann RL. Intlanmation at the neunomuscular junction in myasthenia gravis. Neurology $1991 ; 41: 1497-504$.

Nakagawa T, Miyamoto T. The role of JgG4 as blocking antibodies in asthmatics and in bee keepers. Int Arch Allengy Appl Immunol 1985; 77: 204-5. 


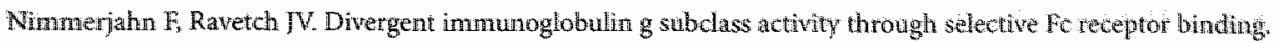
Science $2005 ; 310: 1.510-2$

Wouri-Aria $\mathrm{KT}_{3}$ Wachholz PA, Francis $\mathrm{N}$, Jacobson MR, Walker SM, Wilcock LK, et al Grass pollen immuno-

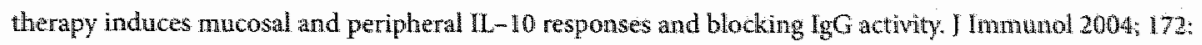
$3252-9$.

Oh Sj. Electromyography : neuromuscular transmission studies. Baltimore; London: Willians \& Wilkins, 1988.

Papanastasiou D, Poulas K, Kokla A, Tzartos SI. Prevention of passively transferred experimental autoimmune myasthenia gravis by Fab fragments of monoclonal antbodies directed against the main immunogenic region of the acetylcholine receptor. T Neuroimmunol 2000; 104: 124-32.

Patrick J, Lindstrom J. Autoimmune response to acetylcholine receptor. Science $1973 ; 180: 871-2$.

Protopapadakis E, Kokla A, Tzartos SI, Mamalaki A. Isolation and dxaracterization of human anti-acetylyoline receptor monoclonal antibodies from transgenic mice expressing human ummunoglobulin loci. Fur J IImmunol 2005; $35: 1960-1968$.

Psaridi-Linardaki L, Mamalaki A, Remoundos M, Tzartos SI. Expression of soluble ligand ... and antibody-binding extracellular domain of human muscle acetylcholine receptor alpha subunit in yeast Pichia pastoris. Role of glycosylation in alpha-bungarotoxim binding. I Biol Chem 2002; 277; 26980-6.

Rauterberg AD, Kohl PK, Hartschuh W, Rauterberg EW. [Complement-independent bulla tormation in penphigus vulgaris by IgC4]. Hautarzt 1988; 39:426-9.

Rey Ex, Zeidel M, Rhine C, Tami J, Krolick K, Fischbach M, et al. Characterization of human anti-acetylcholine receptor momoclonal autoantibodies from the peripheral blood of a myasthenia gravis patient using combinatorial libraries. Clin Immunol 2000; 96: $269-79$.

Richman DP, Agius MA, Kirvan CA, Gomez CM, Fairclough RH, Dupont BL, et al. Antibody effector mechanisms in myasthenia gravis. The complement hypothesis. Ann N Y Acad Sci 1998; 84:1:450-65.

Rock B, Martins CR, Theofilopoulos AN, Balderas RS, Anhalt G), Labib RS, et al. The pathogenic effect of IgG4 autoantibodies in endemic pemphigus foliaceus (fogo selvagem). N Engl I Med 1989; 320: 1463-9.

Rodgard A, Nielsen $\mathrm{IC}$, Djurup R, Somnier F, Gammeltoft S. Acetylcholine receptor antibody in myasthenia gravis: predominance of $\lg$ G subclasses 1 and 3 . Clin Exp Immunol 1987; 67: 82 8.

Schumman J, Van Ree R, Pendok GJ, Van Doom HR. Tan KY, Aalberse RC. Normal human immunoglobulin G4 is bispecific: it has two different antigen-combining sites. Inmunology 1999; 97:693-8.

Scinicariello E. Engleman CW, Jayashankar $\mathbb{L}_{-s}$ McClure HM, Atanasio R. Rhesus macaque antibody moleculcs: sequences and heterogeneity of alpha and gamma constant regions. Immunology $2004 ; 1: 1: 66-74$.

Shearer MH, Dark RD, Chodosh 1 , Kennedy RC. Comparison and characterization of immunoglobulin Gx subclasses among primate species. Clin Diagn Lab Immunol 1999;6:953-8.

Shiono $\mathrm{H}$, Roxanis $\mathrm{I}$, Zhang W, Sims GP, Meager A, lacobson LW, et al. Scemarios for antoimmunization of T and $\mathbb{B}$ cells in myasthenia gravis. Ann $\mathbb{N}$ Y Acad Sci 2003; $998: 237-56$.

Souroujon MC, Pachner AR, Fuchs $S$. The treatment of passively transferred experimental myasthenia with ant idiolypic antibodies. Neurology 1986; $36: 622-5$.

Stassen MH, Machiels BM, Fostieri E, Tzartos SJ, Berrih-Aknin S, Bosmans E, et al. Characterization of a fully human IgGI reconstructed from an anti-AChR Fab. Ann N Y Acad Sci 2003a; 998*399-400.

Stassen MH, Meng F, Melgert E, Machiels BM. Im SH, Fuchs S, ett al. Experimental autommume myasthenia grawis in mice expressing human uimmunoglobulin loci. I Neuroimmunol $2003 \mathrm{~b}_{\mathrm{s}}$ 135: $56 \cdots 61$. 
Tarrab-Hadal R, Aharonow A, Silman 1 . Fuchs $\$$, Abramsky O Experimental autommune myasthenia incuced in nonkeys by purtited acetylcholine receptor. Nature 1975; 256:128-30.

Toyka KV, Brachman DB. Pestronk A, Kao I. Myasthenia gravis: pasisiwe transfer from man to mouse. Science $1975,190: 397-9$.

Toyka KV, Drachman DB, Griffin DE, Pegtrorik A, Winkelstein JA, Fishbeck KH, et al Myasthenia gravis. Study af humoral immune mechanisms by pasive transfer to mice. N Engl J Med 1977; 296:125-31.

Toyka KV, Lowenadler $B$, Heininger $K$, Besinger UA, Birnberger $K L$, Fateh-Moghadam A, et al Passiwely trans ferted myasthenia gravis protection of mouse endplates by Fab fragments from human myasthenic IgG. ) Meurol Neurosurg Psychiatry 1980; $43: 836-40$.

Tuzun $\mathrm{E}_{\mathrm{r}}$ Scott BG, Goluszko $\mathrm{E}$, Higgs $\$$, Christadoss $\mathrm{P}$. Genetic evidence for involvement of classical complement pathway in induction of experimental autoimmune myasthenia gravis. ] Immunol 2003; 171:3847-54.

Tuzun E, Scott BG, Yang $H$, Wu B, Goluszko E, Guigneaux M, t al. Circulating immune complexes augment severity of antibody-mediated myasthenia gravis in lypogammaglobulinemic RIIIS/T mice. I Immunol $2004: 172: 5743-52$.

Tzartos S), Cung MT, Demange $\mathrm{P}$, Loutrari $\mathrm{H}_{3}$ Mamalaki $\mathrm{A}$, Marraud $\mathrm{M}$, et al. "The main immunogenic region (MIR) of the nicotinic acetylcholine receptor and the anti-MIR antibodies. Mol Neurobiol 1991; 5: 1-29.

Tzartos SJ, Lindstrom JM. Monoclonal antibodies used to probe acetylcholine receptor structure: localization of the main immunogenic region and detection of similarities between subunits "Proc Natl Acad Sci U SA $1980 ; 77: 755-9$

Tzartos SJ, Seybold ME, Lindstrom IM. Specificities of antibodies to acetylcholine receptors in sera from myasthenia gravis patients measured by monoclonal antibodies. Proc Natl Acad Sci U S A 1982; 79: 188-92.

van der Zee IS, van Swieten $P_{n}$ Aalberse RC. Serologic aspects of IgG4 antibodies. II. IgG4 antibodies form small, nonprecipitating immune complexes due to functional monowalency. / Immunol 1986; 137: $3566-71$.

van Dijk MA, van de Winkel JG. Human antibodies as next generation therapeutics. Curr Opin Chem Biol 2001; 5: $368-74$.

Vercelli D, De Monte L, Monticelli S, Di Bartolo C, Agresti A. To E or not to E? Can an IL-4-induced B cell choose between IgE and IgG4? Int Arch Allergy Immunol 1998; 116: $1-4$.

Verschuuren JI, Graus YM, Tzartos SI, wan Breda Vriesman PJ, De Baets MH. Paratope- and framework-related cross-reactive ldiotopes on anti-acetylcholine receptor antibodies. J Immunol 1991; 146:941 -8.

Vincent A. Immunology of acetylcholine receptors in relation to myasthenia gravis. Physiol Rev 1980;60:756824

Wakkach A, Poea $S_{0}$ Chastre E, Gespach C. Lecerf F, De La Porte S, et al. Establishment of t hurnan thymic myoid cell line. Phenotypic and functional characteristics. An J Pathol 1999; 155: 1229-40.

Wang ZY, Quia I, Link H. Suppression of experimental autoim mune myasthenia gravis by oral administration of acetylicholine receptor. I Neurommunol 1993; 44: $209-14$.

Wu JM, Wu B, Miagkov A, Adams RN, Drachman DB. Specific immunotherapy of experimental myasthenia grawis in witro: the "guided missile" strategy. Cell Immunol 2001: 208: 137-47.

Zeidel M, Rey E, Tami I, Fischbach M, Sanz I. Genetic and functional characterization of human autoantibodies using combinatorial phage display librartes. Ann N Y Acad Sci 1995; 764: 559-64. 


\section{Chapter 6}

\section{The features of myasthenia gravis with autoantibodies to MuSK}

Dragana Lavrnic, Mario Losen, Ana Vujic, Marc H. De Baets, Ljiljana I. Hajdukovic,

Vidosava Stojanovic, Rajko Trikic, Petar Djukic and Slobodan Apostolski

J Neurol Neurosurg Psychiatry. 2005; 76(8):1099-102 


\section{Abstract}

Objectives: To determine if myasthenia gravis (MG) with antibodies to MuSK is a distinct subgroup of seronegative $\mathrm{MG}$.

Methods: We assayed antibodies to muscle specific tyrosine kinase (MuSK) in $55 \mathrm{MG}$ patients who had no antibodies to acetylcholine receptors and looked for the specific phenotype, comparing clinical features of anti-MuSK positive and anti-MuSK negative $\mathrm{MG}$ patients.

Results: MG with anti-MuSK antibodies was characterised by a striking prevalence of female patients ( 15 women, two men). Age at onset ranged from 22 to 52 years, with $70.6 \%$ of patients presenting at $<40$ years of age. The majority of patients $(82.4 \%)$ had prevalent involvement of facial and bulbar muscles. One third of them did not respond well to anticholinesterase drugs. Steroid immunosuppression was effective in eight patients (44.4\%). Nine patients underwent thymectomy; six of these had no thymus pathology, while three had a hyperplastic thymus. At the end of the observation period, six (35.3\%) patients were in remission, five (29.4\%) improved, four (23.6\%) did not change, and two (11.7\%) had died.

Conclusions: MG patients with antibodies to MuSK have characteristic clinical features that are different from features of the remaining seronegative MG patients. This emphasises the predictive value of anti-MuSK antibody analysis in seronegative MG patients.

\section{Introduction}

Myasthenia gravis (MG) is an autoimmune disorder of the skeletal muscles characterised by a decrease in the number of available acetylcholine receptors ( $A C h R$ ). Approximately $80-90 \%$ of patients have detectable serum anti-AChR antibodies, ${ }^{1}$ but there is conclusive evidence that patients without these antibodies also have an antibody mediated disorder. ${ }^{2}$ Recently, it was shown that approximately $40-50 \%$ of seronegative MG (SNMG) patients have antibodies to a surface membrane enzyme, muscle specific tyrosine kinase (MuSK), which is responsible for agrin induced $A C h R$ clustering at the postsynaptic membrane." A specific clinical phenotype of SNMG has been described in patients with anti-MuSK antibodies, ${ }^{4}$ but these antibodies have been also detected in seropositive MG with or without thymoma." We analysed the clinical, electrophysiological, pharmacological, and pathological features in 17 SNMG patients with anti-MuSK antibodies. 


\section{Methods}

More than 1200 patients with MG have been treated at the Institute of Neurology, Clinical Centre of Serbia, in Belgrade. In all, the diagnosis of MG was based on a typical clinical pattern, a positive neostigmine and/or edrophonium test and decreasing response on repetitive nerve stimulation (performed on the facial nerve-nasal muscle and axillary nerve-deltoid muscle systems) and/or increased jitter in single fibre EMG. During the past 2 years anti-AChR antibodies were analysed in the sera of 276 patients: 221 (80.1\%) patients were positive and 55 (19.9\%) were negative. Recently, we assayed anti-MuSK antibodies in all seronegative MG patients; 40 women and 15 men. At the time these patients were diagnosed and treated we were unable to perform either anti-AChR Ab or anti-MuSK Ab analyses, thus we did not know the patients' antibody status. Consequently, all the patients were treated according to the clinical form of the disease, regardless of the presence of antibodies.

The severity of MG was examined at the peak of the disease and graded by using the modified Osserman's scale and the modified Besinger's standardised clinical score. ${ }^{6}$ Four degrees of muscle weakness were determined. Full muscle strength was graded 0 , fatigability 1 , moderate to severe weakness 2 , and total loss of function 3 . The severity of the disease was expressed as the mean disability score (MDS), which was obtained by adding the scores for each of the nine examined muscle groups (extraocular, jaw, facial, bulbar, neck, respiratory, proximal and distal upper limb muscles, and proximal lower limb muscles). In every patient, the MDS was determined at the peak of disease (MDS1) and at the end of the follow up period (MDS2). The outcome was expressed by the mean disability score quotient (MDSQ), which was obtained by MDSQ = MDS2/MDS1. An MDSQ between 0.5 and 1 indicated no improwement, while values between 0 and 0.5 were considered as improvement. Patient was considered to be in remission when MDSQ was 0. Pharmacological remission was defined as the absence of any myasthenic signs with the therapy, and complete remission the absence of any myasthenic signs without any therapy.

The clinical characteristics of anti-MuSK positive patients were compared with those of anti-MuSK negative SNMG patients. All patients have been followed longitudinally for 3-10 years.

The concentration of anti-AChR antibodies was measured by a standard radioimmunoassay method using $A C h R$ radioimmunoassay kits (CIS Biointernational). Anti-MuSK antibodies were detected using the MuSK Ab Assay kit (RSR Ltd, Cardiff, UK), according to the manufacturer's protocol. Briefly, $5 \mu \mathrm{L}$ of serum was incubated with $50 \mu \mathrm{L}$ of ${ }^{125} \mathrm{I}-\mathrm{MuSK}$ (extracellular domains) for 16 hours. Human antibodies were immunoprecipitated for 2 hours, centrifuged, and the washed pellets were measured in a gamma counter.

Statistical analysis. For descriptive presentation of numerical and attributive variables, absolute and relative frequencies were calculated. The numerical variables are presented as means (SD). The statistical evaluation of the data was performed using SPSS ver sion 10.0 for Windows. A $\chi^{2}$ test with Yates's correction was used to compare the frequency distribution of different gradations of categorical variables in the group of patients with 
and without anti-MuSK antibodies, and also to compare the difference in the frequency distribution of the same variable between these two groups of patients. Values of $\mathrm{p}<0.05$ were considered to be statistically significant.

\section{Results}

Among the 55 SNMG patients, 17 were anti-MuSK antibody positive (30.9\%) and 38 $(69.1 \%)$ were anti-MuSK Ab negative. In the group of patients with anti-MuSK antibodies there was a striking prevalence of females. Fifteen patients were women ( $88.2 \%)$ and only two $(11.8 \%$ ) were men (female:male ratio $7.5: 1)\left(\chi^{2}\right.$ with Yates's correction $=8.4$, d.f. $=1$, $\mathrm{p}<0.01)$. Among anti-MuSK negative SNMG patients there was also a female preponderance ( 24 females $(63.2 \%$ ) and 14 males ( $36.8 \%$ ); female:male ratio $1.7: 1$ ), but without statistical significance ( $\chi^{2}$ with Yates's correction $=2.2$, d.f. $=1, p>0.05$ ). However, the difference in sex distribution between anti-MuSK positive and anti-MuSK negative patients was of no statistical significance ( $\chi^{2}$ with Yates's correction $\left.=2.4, \mathrm{~d} . \mathrm{f}=1, \mathrm{p}>0.05\right)$. The age at onset of the anti-MuSK positive MG patients ranged from 22 to 52 years (mean (SD (35.6 (10.3) years); in 12 of 17 patients ( $70.6 \%$ ), onset of the disease was before the age of 40 years. The age at onset of the disease in anti-MuSK negative patients was similar: 38.3 (12.8) years (range 14 to 63 years).

In nine of 17 anti-MuSK positive MG patients (52.9\%), the first symptoms of the disease were oculobulbar, in three (17.6\%) bulbar, in two (11.8\%) ocular, and in the remaining three $(17.6 \%)$ generalised $(2=7.3$, d.f. $=3, p>0.05)$. At the peak of the disease, 14 antiMuSK positive patients ( $82.4 \%$ ) had a severe generalised form of MG (IIB), and only three patients $(17.6 \%)$ had the mild generalised form (ILA) of the disease (Figure 1). The majority of the patients shared a similar pattern of muscle weakness, with prevalent involvement of facial and bulbar muscles (in 14 of 17 patients; 82.4\%). Six patients (35.3\%) developed

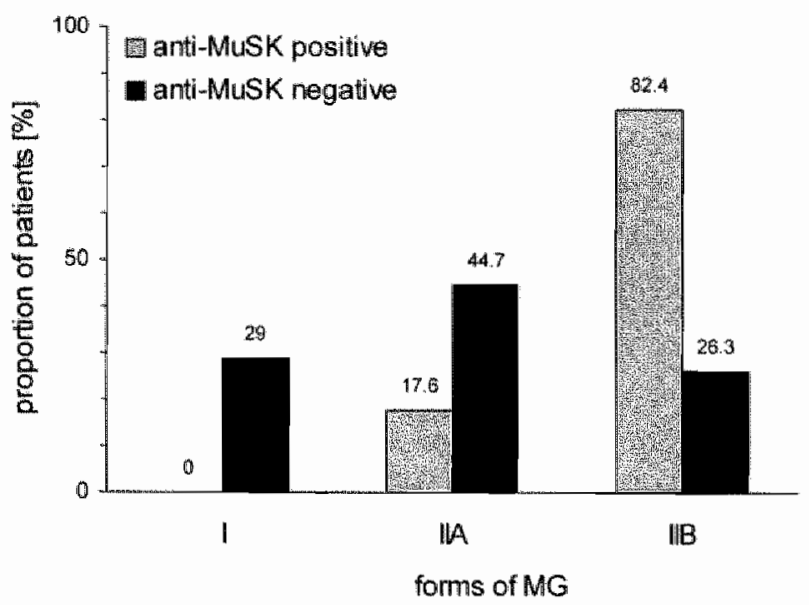

Figure 1. MG forms (according to Ossermans' classification) in SNMG patients. $p<0.01$. 
respiratory muscle weakness and underwent endotracheal intubation with artificial ventilation. In anti-MuSK negative SNMG patients, 11 patients (29\%) had the ocular form (1) at the peak of the disease, $17(44.7 \%)$ had the mild generalised form (IIA), and 10 (26.3\%) had the severe generalised form (IIB), according to Osserman's classification (Figure 1). By contrast, anti-MuSK positive patients had more severe forms of the disease $(2=15.8$, d. $f=2$, $\mathrm{p}<0.01)$. In the chronic phase of the disease, three anti-MuSK positive patients $(17.6 \%) \mathrm{de}-$ veloped severe atrophy of facial and lingual muscles, while the same feature was observed in only one anti-MuSK negative patient $(2.6 \%)\left(\chi^{2}\right.$ with Yates's correction $=2.15$, d.f. $=1$, p $>0.05$ ). Despite the fact that all patients had generalised muscle weakness, a decreasing response on repetitive nerve stimulation was registered in only eight anti-MuSK positive patients (47.1\%) (Figure 2). Single fibre EMG was performed in 11 patients and was positive in all (100\%). In anti-MuSK negative SNMG patients, decreasing response on repetitive nerve stimulation was registered in 23 of 27 patients $(85.2 \%)$ with generalised forms of the disease (Figure 2). The sensitivity of repetitive nerve stimulation was significantly lower in anti-MuSK positive patients ( $\chi^{2}$ with Yates's correction $=5.6, \mathrm{~d} . \mathrm{f}_{\mathrm{n}}=1, \mathrm{p}<0.05$ ).

The effect of neostigmine or edrophonium was negative or equivocal in five anti-MuSK positive patients (29.4\%). Five patients (29.4\%) had hypersensitive response to usual single dose of pyridostigmine ( $60 \mathrm{mg}$ ) (Figure 2). This hypersensitivity was manifested by severe muscle fasciculations, especially in the ocular and facial muscles, blurred vision, hypersalivation, and abdominal cramps. In anti-MuSK negative SNMG patients, hypersensitive response to pyridostigmine was observed in only one patient $(2.6 \%)\left(\chi^{2}\right.$ with Yates's correction $=6.0, \mathrm{~d} . \mathrm{f} .=1, \mathrm{p}<0.05$ ) (Figure 2). Owing to hypersensitive response to pyridostigmine, all these patients were treated with very small, divided doses of $10-30 \mathrm{mg}$ at every 4-8 hours. The remaining patients received pyridostigmine as doses of $60 \mathrm{mg}$ every $4-8$ hours. In addition to anticholinesterase drugs, all anti-MuSK positive and 35 anti-MuSK negative (92\%) patients were also treated with corticosteroid drugs (average dose $60 \mathrm{mg}$ of pred-

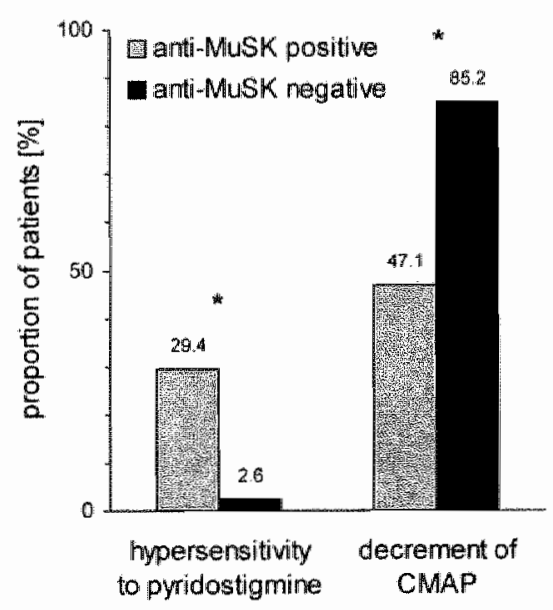

Figure 2. Hypersensitivity to pyridostigmine and decremental response of the compound muscle action potential after repetitive nerve stimulation in SNMG patients. $p<0,05$. 
nisone on alternate days). In the patients who did not respond well to the treatment with anticholinesterases and corticosteroids, additional immunosuppressive drugs were added. Azathioprine was administered in five anti-MuSK positive $(29.4 \%)$ and 14 anti-MuSK negative (36.8\%) patients. Three anti-MuSK positive (17.6\%) and one anti-MuSK negative $(2.6 \%)$ patient were treated with cyclosporine $A$, and one anti-MuSK positive patient (5.9\%) was treated with mycophenolate mofetil. In the group of eight anti-MuSK positive patients successfully treated with corticosteroid drugs, two were in complete remission, four were in pharmacological remission, and two improved significantly. In the group of anti-MUSK positive patients treated with a combination of immunosuppressive drugs, two patients had to be treated additionally with intravenous immunoglobulin and four with plasma exchange because of an acute exacerbation and respiratory crisis. Among them, five patients improved but one died at 56 years of age, 8 years after the onset of the disease. Thymectomy was performed in nine of 17 (52.9\%) anti-MuSK positive MG patients. The criteria for thymectomy were the generalised form of the disease and age $<50$ years. None of the patients had a thymoma. A hyperplastic thymus was found in three patients $(17.6 \%)$, aged 23,32 , and 48 years, and a normal or atrophic thymus gland in the remaining six. In the group of anti-MUSK negative SNMG patients, thymectomy was performed in 17 of 27 patients with generalised forms of the disease (63\%). Thymoma was found in two (11.8\%), hyperplastic thymus in seven (41.2\%), and atrophic or persistent thymus gland in eight ( $47 \%)$.

At the end of the follow up period, two anti-MuSK positive patients (11.8\%) were in complete remission, four (23.5\%) were in pharmacological remission, five (29.4\%) had improved, four (23.5\%) had an unchanged condition, and two patients had died $(2=2.2$, d.f. $=4, p>0.05$ ) (Figure 3). One patient died from a myasthenic crisis at the age of 56 years and the other, aged 24 years, from an acute intestinal necrosis. The latter patient was diagnosed 2.5 months prior to her death, and she received corticosteroids in dosage $20 \mathrm{mg}$ daily for 15

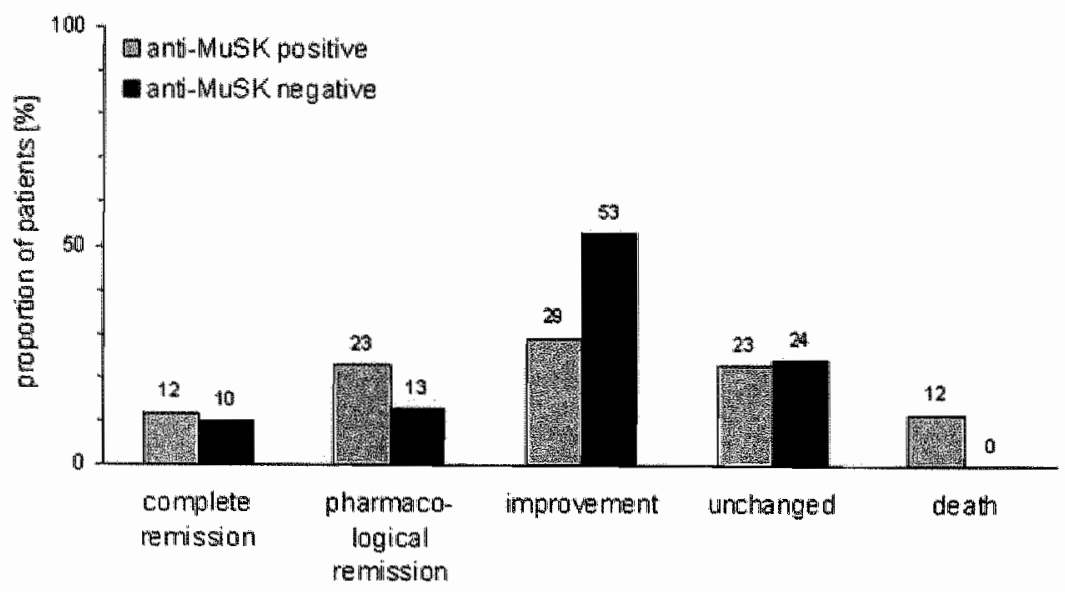

Figure 3. The outcome of MG in SNMG patients. $p>0.05$. 
days and then $40 \mathrm{mg}$ daily. She was not thymectomised. She had associated thrombophilia, which was probably the cause of the lethal acute intestinal necrosis. Among the thymectomised patients, two achieved complete remission, two were in pharmacological remission; one significantly improved, three had an unchanged condition, and one died. Among the anti-MuSK negative SNMG patients, at the end of the follow up period, four (10.5\%) paw tients were in complete remission, five (13.2\%) in pharmacological remission, 20 (52.6\%) had improved, and nine (23.7\%) had an unchanged condition (Figure 3 ). There was no statistically significant difference in the outcome of the disease between the anti-MuSK positive and negative patients $(2=6.8$, d.f. $=4, p>0.05)$.

We noticed associated disorders in nine (53\%) anti-MuSK positive MG patients: systemic lupus erythematosus (two patients), myoma uteri (three), allergy to penicillin (one), duodenal ulcer (one), carcinoma (one), struma nodosa (one), and thrombophilia (one), Among the SNMG anti-MuSK negative patients, associated diseases were found in seven (18.4\%) patients: hyperthyreosis (four patients), lung cancer (one), struma nodosa (one), bronchial asthma (one), and pulmonary fibrosis (one). The occurrence of associated diseases was significantly higher in the anti-MuSK positive patients ( $\chi^{2}$ with Yates's correction $=5.4, \mathrm{~d} . \mathrm{f} .=1, \mathrm{p}<0.05)$. In contrast, there was no statistically significant difference in the frequency of each disorder, either in the group of anti-MuSK positive patients or between the anti-MuSK positive and anti-MuSK negative SNMG patients $(\mathrm{p}>0.05)$.

\section{Discussion}

We detected anti-MuSK antibodies in 17 of $44(38.6 \%)$ our patients with generalised SNMG. The frequency is lower than the $40-70 \%$ frequency reported in other studies of white patients, ${ }^{3,4,7-10}$ and significantly higher than the $4 \%$ reported in Chinese patients. ${ }^{11}$ The female preponderance was the significant feature of our anti-MuSK positive MG patients, which was in accordance with the finding in other studies of white patients, ${ }^{4,} \gamma_{i}, v_{0}, 10$ but not with the study in Chinese patients, 11 nor in the study published by Zhou et al.1. In $70 \%$ of our patients, the onset of the disease was before the age of 40 years (young onset MuSK-MG). Similar results (57\%) were published by Evoli et al. ${ }^{4}$ We found predominant involvement of facial and bulbar muscles in $82.4 \%$ of patients, similar to papers reporting prevalent oculobulbar weakness in anti-MuSK positive MG patients.,3,4,9.10 The weakness of masticatory, facial, and lingual muscles characterised the majority of our patients, and in the chronic phase of the disease, some of these patients (17.6\%) developed severe atrophy of these muscles. A high frequency of respiratory crisis (35.3\%) was similar to observations reported by other groups. ${ }^{3}$ The diagnostic value of neostigmine or edrophonium test and repetitive stimulation was low in our patients. Incomplete response to anticholinesterase drugs has been reported by several groups, ${ }^{3,4,7.8}$ but we observed hypersensitive reaction to average doses of oral pyridostigmine with exacerbation of muscle weakness and cholinergic signs in almost $30 \%$ of patients. Almost half of the patients $(47 \%)$ responded to corticosteroid immunosuppression, while the others needed to be treated with a combination 
of immumosuppressive drugs and with an intermittent plasma exchange or intravenous immunoglobulin. Most papers reported no benefit from thymectomy in anti-MuSK positive MG patients, ${ }^{4,78}$ but we observed significant improvement or remission in more than a half of thymectomised patients. We also noticed better response to corticosteroid treatment in thymectomised patients. The majority had no thymus pathology, but three of nine thymectomised patients had a hyperplastic thymus. In spite of the fact that our patients with anti-MUSK antibodies and MG have specific clinical, electrophysiological, pharmacological, and pathological features, we still do not know if these antibodies are the primary cause of the disease. ${ }^{13}$

\section{Conclusions}

Our results indicate that MG with antibodies to MuSK has a different phenotype from the remaining seronegative MG. MG with anti-MuSK antibodies was characterised by a striking prevalence of females $(7.5: 1)$, severe, predominantly faciobulbar weakness $(82.4 \%)$, and more frequent occurrence of facial and lingual muscle atrophy (17.6\%). Anti-MuSK MG patients frequently develop hypersensitivity to anticholinesterase drugs $(29.4 \%)$. Thymus pathology is less common in this subgroup of patients, but thymectomy is still beneficial. Routine repetitive nerve stimulation test has a low, while single fibre EMG has very high sensitivity. The specific clinical, electrophysiological, and immunological presentation, the thymus pathology, and the therapeutic response, particularly to anticholinesterase drugs, implicate that MG with anti-MuSK antibodies is a specific subgroup of seronegative MG. This emphasises the predictive value of anti-MuSK antibody analysis in all seronegative MG patients.

\section{References}

1. Lindstrom, J.M., Acetylcholine receptors and myasthenia. Muscle Nerve, 2000, 23(4): p. 453-77.

2. Burges, J. A. Vincent, P.C. Molenaar, J. Newsom-Davis, C. Peers, and D. Wray, Passivetransfer of seronegative myasthenia gravis to mice. Muscle Nerve, 1994. 17(12): p. $1393-400$.

3.Hoch, W. I. McConville, S. Felms, J. Newsom-Davis, A. Melms, and A. Vincent, Auto-antibodies to the receptor tyrosine kinase MuSK in patients with myasthenia gravis without acetylcholine receptor antibodies. Nat Med, 2001. 7(3): p. 365-8.

4. Evoli, A., P.A. Tonali, L. Padua, M...... Monaco, F Scuderi, A.P. Batocchi, M. Marino, and E. Bartoctioni, Clinical correlates with anti-MUSK antibodies in generalized seronegative myastheria gravis. Brain, 2003. 126 (Pt 10): p. 2304-11.

5. Ohta, K., K. Shigemoto, S. Kubo, N. Maruyama, Y. Abe, N. Ueda and M.Ohta, MuSKantibodies in AChR Abseropositive MG vs AChR Ab-seronegative MG. Neurology, 2004. 62(11): p. 2132-3.

6. Besinger, U.A., K.V. Toyka, M. Homberg, K. Heininger, R. Hohlfeld, and A. Fateh-Moghadan, Myasthenia gravis: long-term correlation of binding and bungarotoxin blocking antibodies against acetylcholine receptors with changes in disease severity. Neurology, 1983. 33(10); p. 1316-21. 


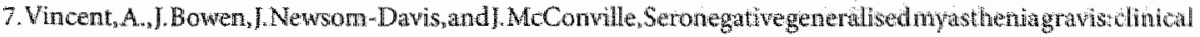
features, antibodies, and their targets. Lancet Newrol, 2003. 2(2); p.99-106.

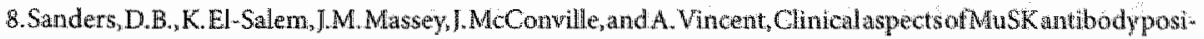
tive seronegative $\mathrm{MG}$. Neurology, 2003. 60(12). p, $1978-80$.

9. Scuderi, F. M. Marino, L.Colonna, F.Mannella, A Evoli, C. Provenzano, and E, Bartoccioni, Anti-p 110autoantibodies identify a subtype of "seronegative" myasthetia gravis with prominent oculobulbar irmolwetment. Lab Invest, 2002. 82(9): p. 1139-46.

10. McConville, J.M.E. Farrugia, D. Beeson, U. Kishore, R. Metcalfe, J Newson-Davis, and A. Vincent, Detection and characterization of MUSK antibodies in seronegative myasthenia gravis. Ann Neurol, 2004. 55(4): p. $580-4$

11. Yeh, J.H., WH. Chen, H.C. Chiw, and A. Vincent, Low frequency of MuSKantibody in generalized seronegative myasthenia gravis among Chinese. Neurology, 2004.62(11);p, $2131-2$.

12. Zhou, L. J. McConville, V. Chaudhry, R.N. Adams, R.L. Skolasky, A Vincent, and D.B. Drachman, Clinical comparison of muscle-specific tyrosine kindse (MuSK) antibody-positive and -negative myasthenic patients. Muscle Nerve, 2004. 30(1): p. 55-60.

13. Selcen, D., T. Fukuda, X.M. Shen, and A.G. Engel, Are MuSK antibodies the primary cause of nyasthenic symptoms? Neurology, $2004.62(11):$ p. 1945-50. 


\section{Summary and general discussion}

The postsynaptic membrane of the neuromuscular junction (NMJ) is a highly specialised structure that initiates muscle contraction after activation by the neurotransmitter acetylcholine. Because control of movement is vitally important, the NMJ has been subject to many independent targeted attacks in the course of evolution. Toxins against neuromuscular proteins are used both as a weapon and defence and well known examples include cobra-toxin, curare and botulinum-toxin. The similarity of symptoms caused by these toxins compared to congenital and acquired disorders of muscle movement has helped to find the cause of human diseases involving the NMJ. The first treatment for the muscle weakness of myasthenia gravis (MG) patients was the acetylcholine esterase inhibitor physostigmine, an antidote against curare (Jolly, 1895; Walker, 1934). However, treatment effects of acetylcholine esterase inhibitors are only short lasting and incomplete. Thymectomy was introduced as a treatment for $M G$ following the observation of thymic abnormalities such as thymomas and follicular hyperplasia during autopsies of MG patients (Starr, 1912). Thymectomy proved to be beneficiall for some MG patients. The discovery of Patrick and Lindstrom in 1973 that MG is caused in $85 \%$ of patients by anti-AChR antibodies attacking the NMJ led to the development of immunosuppression and plasmapheresis therapies (Patrick and Lindstrom, 1973). The latter treatments are efficacious in most $M G$ patients, including those without anti-AChR antibodies, albeit with occasional severe side effects. Intravenous immunoglobulin (IVIg) has also successfully been used to treat MG (Fateh-Moghadam et al., 1984). Unfortunately, none of the aforementioned treatments, alone or in combination, is sufficient to treat all MG patients. Particularly the treatment of $M G$ patients without anti-AChR antibodies is difficult because the pathogenesis is not well understood. The recent discovery of anti-MuSK antibodies in some, but not all patients without anti-AChR antibodies (Hoch et al., 2001), has shown that MG is a heterogeneous disease with different causes and varying responses to treatment. Hence, more efficient and specific treatments are required and the subtypes of MG need further characterisation. For the analysis of MG, not only the changes of the immune system, but also the response of the muscle is of particular interest. The molecular composition of the neuromuscullar junction determines the susceptibility to the autoantibody attack. The interaction of the $\mathrm{AChR}$ with rapsyn is pivotal for the integrity of the postsynaptic membrane. Gene therapy to increase synaptic rapsyn expression facilitated enhanced resistance and recovery of the treated muscles (Chapters 2 and 3 ). Because also adverse effects of extrasynaptic rapsyn expression in ongoing chronic EAMG were observed, further research is needed to ensure endplate specific rapsyn expression and to establish safe and efficient gene transfer protocols for muscle therapy. 
An alternative therapeutic approach that could potentially be realised with the available biotechnology on a short term is the treatment with neuroprotective recombinant human antibodies (Chapter 5). For this purpose a human IgG4 specific for the AChR was produced. Because this IgG4 can compete with pathogenic IgGl anti-AChR autoantibodles, and does not by itself damage the postsynaptic membrane, it prevented the disease in a non-human primate model.

Both aforementioned experimental therapies are only applicable to $\mathrm{MG}$ with antiAChR antibodies. MG patients with anti-MuSK antibodies have normal AChR levels (Selcen et al., 2004) and a high proportion of IgG4 autoantibodies (Hoch et al., 2001). AntiMuSK antibodies do not aftect all muscles equally, and the identification of the underlying differences between affected and resistant endplates could help to find targets for therapy. The available diagnostic method for the analysis of anti-MuSK antibodies is an important step for evaluating the efficacy of conventional therapies (Chapter 6). Moreover, it will be important to mimic the clinical effect of these antibodies in an animal model.

Chapter 1 reviews the use of experimental models for MG. Experimental autoimmune myasthenia gravis (EAMG) can be induced in a large number of animal species by active immunisation with $A C h R$, by passive transfer of anti-AChR antibodies, by autologous bone marrow transplantation and cyclosporin, or occur spontaneously. Depending on the model used, different immunological mechanisms are operational. In the active immunisation model, the $\mathrm{T}$ cell is pivotal in directing the anti-AChR antibody production towards pathogenic, that is, cross-linking and complement-fixing antibodies. Injection of anti-AChR antibodies alone suffices to induce EAMG, excluding the role of specific cellmediated immune responses in the effector phase of the disease. Aged rats are resistant to the induction of EAMG by passive transfer and active immunisation. This resistance correlates with increased amount of rapsyn and s-laminin (laminin $\alpha 1 \beta 2 \gamma 1$ ) relative to the AChR at the neuromuscular junction. In bone marrow transplantation and cyclosporin EAMG, a dysregulation of the immune system in the absence of immunisation is capable of inducing myasthenia.

Chapter 2 describes the experimental approach to study the role of the postsynaptic protein rapsyn in the stabilisation of the AChR in EAMG. Because the AChR is clustered and anchored in the postsynaptic membrane of the neuromuscular junction by rapsyn, we hypothesised that endogenous rapsyn expression may be an important determinant of AChR loss and neuromuscular transmission failure in the human disease and that upregulation of rapsyn expression could be used therapeutically. To examine frrst a potential therapeutic application of rapsyn upregulation, we induced acute EAMG in young rats by passive transfer of $\mathrm{AChR}$ antibody, $\mathrm{mAb} 35$, and used in wivo electroporation to over-express rapsyn unilaterally in one muscle. We looked at the compound muscle action potentials, at rapsyn and AChR expression by quantitative radioimmunoassay and immunofluorescence, and at the morphology of the NMJs, comparing between the electroporated and untreated muscles, as well as between control and EAMG rats. In control rats transfected 
muscle fibres had extrasynaptic rapsyn aggregates, as well as slightly increased rapsyn and AChR concentrations at the NMJ. In EAMG rats, despite deposits of the membrane at tack complex, the rapsyn-overexpressing muscles showed no decrement in the compound muscle action potentials, no loss of $A C h R$, and the majority had normal postsynaptic folds, whereas endplates of untreated muscles showed typical AChR loss and morphological damage. These data suggest that individual differences in innate rapsyn expression could be the key factor in determining disease severity, more than the concentration of circulating anti- $A C h \mathbb{R}$ antibodies.

In Chapter 3 the potential of rapsyn overexpression as a therapy for ongoing chronic EAMG is investigated. Despite of the presence of high titres of anti-AChR antibodies, the rapsyn overexpression resulted in an increase of total membrane $A C h R$ levels in ongoing chronic EAMG compared with the contralateral untreated muscles. This increased AChR was localised at endplates and in extrasynaptic membrane aggregates, and bound by rat anti-AChR antibodies. Endplates in transfected fibres of EAMG muscles with a high level of rapsyn recovered from $A C h R$ loss and were comparable to endplates from healthy animals, but the average AChR level at the neuromuscular junctions was not changed compared to the contralateral untreated muscles. At the ultrastructural level, a large number of endplates in rapsyn-treated muscles had an increased damage of the postsynaptic membrane. The results suggest that extrasynaptic rapsyn might interfere with synapse recovery in EAMG and possibly MG, while endplate-specific upregulation of rapsyn expression might be therapeutically helpful.

Chapter 4 reviews the role of non-human primate studies in the development of therapies for the treatment of MG. The phylogenetic proximity between higher primate species and humans is reflected by a high degree of immunological similarity. Non-human primates are therefore important experimental models for immune-based diseases in the human population. Non-human primate disease models are becoming increasingly important for the preclinical development and efficacy evaluation of new therapies, in particular those based on biological molecules, which by their high specificity cannot be tested in rodents. For the research of $M G$, active immunisation with Torpedo $A C h \mathbb{R}$ and passive transfer of human autoantibodies have been used. Active immunisation causes a severe disease and can lead to a high rate of respiratory faulure and death. Per contra, passive immunisation leads to milder and reversible MG symptoms and is therefore more suitable for the study of antibody effects on the neuromuscular junction.

Chapter 5 describes a therapeutic approach for the protection of the $A C h \mathbb{R}$ against a monoclonal human auto-antibody in rhesus monkeys. The human antigen binding fragment Fab 637, which is specific for the human acetylcholine receptor (AChR), protects against loss of $A C h R s$ by blocking the binding of pathogenic $M G$ autoantibodies in vitro. Fab 637 was used for the production and analysis of whole human IgG1 637 and IgG4 637. These antibodies bound to the human AChR with high affinity. Immunohistochemical. studies showed that the antibodies cross-reacted with the muscle AChR of rhesus mon- 
keys, but not from lower primates. IgG1 637 bound and activated complement, whereas IgG4 637 did not. Both recombinant antibodies were bivalent and induced degradation of AChR of TE671 cells by antigenic modulation. The pathogenicity of the antibodies was tested in a passive transfer experimental MG model with rhesus monkeys. IgGI 637 induced muscle weakness and a decremental response of the muscle compound action potential after repetitive nerve stimulation. IgG4 637 did not have any pathogenic effect when injected, even at high concentrations tested. Thus, cross-linking of the AChR is not sufficient and complement binding is essential for induction of the disease. The induction of the disease was prevented in animals that received IgG4 637 prior to the challenge with IgG1 637 . The results indicate that IgG1 anti-AChR antibodies are sufficient to induce muscle weakness and IgG4 can prevent the pathogenic effects of IgGl autoantibodies in non human primates. These studies suggest a novel approach for the development of a treatment of patients suffering from $M G$.

In Chapter 6 the effect of anti-MuSK antibodies on the clinical manifestation of seronegative patients were studied by comparing clinical features of anti-MuSK positive and anti-MuSK negative MG patients. The aim was to determine if myasthenia gravis (MG) with antibodies to MuSK is a distinct subgroup of seronegative MG. Antibodies to muscle specific tyrosine kinase (MuSK) were assayed in $55 \mathrm{MG}$ patients who had no antibodies to acetylcholine receptors. MG with anti-MuSK antibodies was characterised by a striking prevalence of female patients ( 15 women, two men). Age at onset ranged from 22 to 52 years, with $70.6 \%$ of patients presenting at $<40$ years of age. The majority of patients (82.4\%) had prevalent involvement of facial and bulbar muscles. One third of them did not respond well to anticholinesterase drugs. Steroid immunosuppression was effective in eight patients (44.,4\%). Nine patients underwent thymectomy; six of these had no thymus pathology, while three had a hyperplastic thymus. At the end of the observation period, six (35.3\%) patients were in remission, five $(29.4 \%)$ improved, four $(23.6 \%)$ did not change, and two (11.7\%) had died. Hence, MG patients with antibodies to MuSK have characteristic clinical features that are different from those of the remaining seronegative $\mathrm{MG}$ patients. This emphasises the predictive value of anti-MuSK antibody analysis in seronegative MG patients.

The disease mechanisms in MG with anti-MuSK antibodies are not yet understood. One of the key questions is if complement mediated damage is also involved in this disease. The high titres of IgG4 anti-MuSK autoantibodies (McConville et al., 2004) and the intact morphology of endplates found in biopsies (Selcen et al., 2004) suggest that the effector mechanism is entirely different from $M G$ with anti-AChR antibodies. It would be of great value to isolate monoclonal anti-MuSK autoantibodies and to reproduce the patients' clinical features in an animal model.

The effect of AChR antibodies has been analysed in detail using the experimental autoimmune $M G$ animal model. In combination with the aforementioned results, there is accumulating evidence that the changes of the postsynaptic membrane after binding of anti-ACh $R$ are the result of a dynamic interaction of the immune system with the muscle. 
The muscle actively enhances AChRs turnover and removes AChRs from the cell-menbrane that are cross-linked by antibodies (antigenic modulation). This effect of anti-AChR antibodies is insufficient to cause destruction of the NMJ by itself (Chapter 5) and it is not yet clear if it is an essential step of the following steps of the pathogenesis. When human IgG1 or IgG3 bind to AChRs at the NM], the antibodies are further cross-linked by the complement molecule Clq. Also this step does not cause the disease directly, but it mediates the activation of the classical cascade of the complement system leading to the insertion of membrane attack complexes (MAC) into the postsynaptic membrane (Tuzun et al., 2003). The membrane attack complex is a transmembrane pore that causes loss of cytoplasmic proteins and depolarisation of the membrane. It is intended for killing pathogens, but upon auto-antibody binding, MAC can also cause depolarisation of the postsynaptic membrane (Mozrzymas et al., 1993). However, nucleated cells can remove autologous complement from the membrane by vesiculation of MAC containing membranes. Because endocytosis is involved in AChR degradation (Kuncl et al., 1993), it is possible that this mechanism is responsible for the loss of postsynaptic membrane folding and $A C h R$ loss (Morgan, 1989). The degree of MAC induced AChR loss and postsynaptic membrane damage strongly depends on the anchoring of membrane proteins to the cytoskeleton and the basal lamina. The $\mathrm{AChR}$ is clustered in the membrane by rapsyn which, in turn, is linked to the cytoskeleton via $\beta$-dystroglycan, utrophin and F-actin (Chapter 1, Figure 1 , page 21 ). Moreover, $\beta$-dystro-

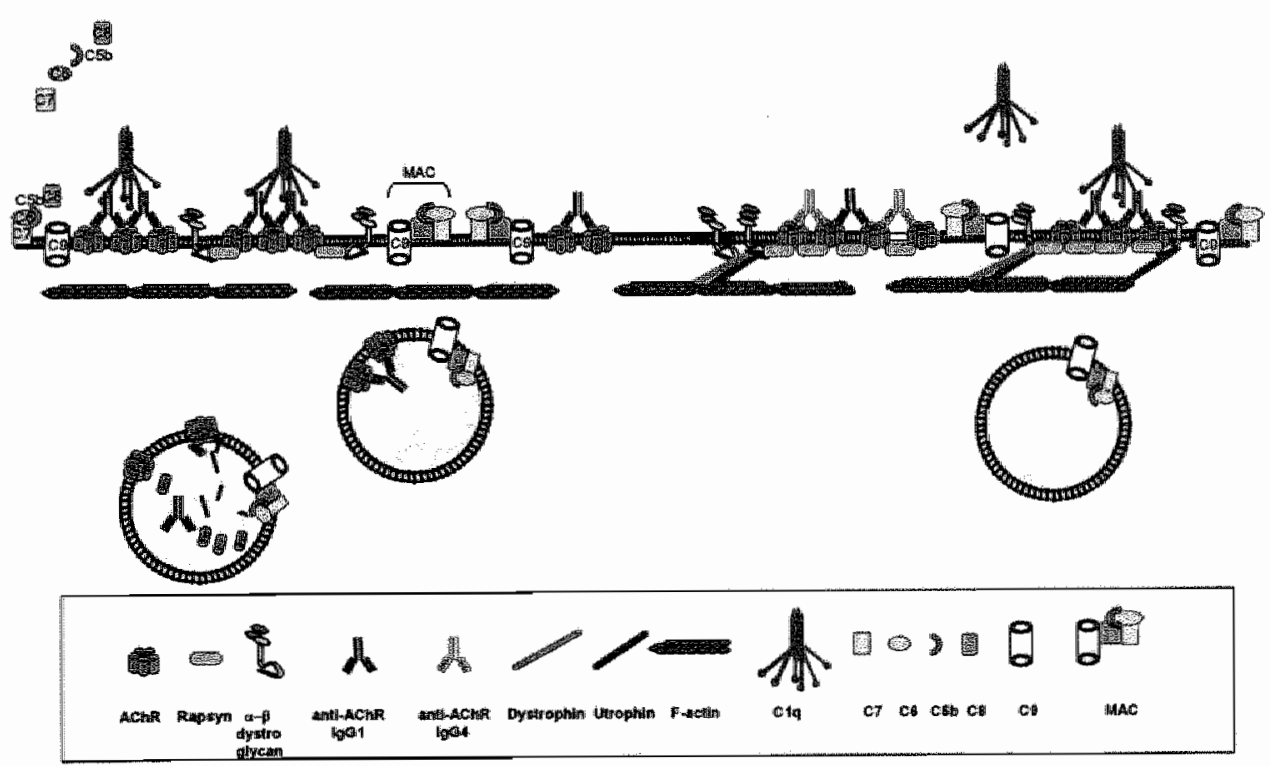

Figure 1. Hypothetical scheme of complement induced degradation of the postsynaptic membrame. Left: The AChR is weakly anchored to the cytoskeleton by rapsyn, dystrogliycan and utrophin and internalised by the muscle after complement activation. Right; postsynaptic proteins are protected by lig 4 and stabilised by anchoring to the cytoskeleton. Complement is removed from the membrane, but without concomitant loss of $A C h R$. 
glycan is linked to the basal membrane via a-dystroglycan and s-laminin (Bezakova and Ruegg, 2003). Increased rapsyn expression stabilises the NMJ and increases the resistance to complement (Figure 1). Rapsyn expression determines the degree of destruction at the NM] after antibody binding to the AChR (Chapter 2). This hypothesis can explain why some muscles are more affected than others in MG patients. Similarly, differences in rapsyn expression could influence the severity of $M G$ and explain why anti-AChR antibody titres do not generally correlate well with clinical symptoms. Therefore, it will be interesting to study if rapsyn expression is influenced by promoter polymorphisms and how the rapsyn promoter is regulated by transcription factors such as Kaiso in different muscles and individuals.

Increased rapsyn expression does not only protect but can help to recover the $A C h \mathbb{R}$ levels of the damaged NMJ in ongoing EAMG (Chapter 3). In the presence of autoantibodies, only synaptic expression of rapsyn increases AChR lewels in the postsynaptic membrane. For this reason, it will be interesting to study the therapeutic effect of rapsyn expression under the control of the endplate specific promoter of the AChR $\varepsilon$-subunit.

Because anti-AChR antibodies do not directly damage the NMI, but elicit a pathogenic effect wia the $M A C_{\text {, }}$ the prevention of complement binding to the endplate could potentially treat MG. Human IgG4 does not bind complement, and AChR specific IgG4 blocks the binding of IgG1 (and likely IgG3) auto-antibodies (Chapter 5). This effect is potentially therapeutic at 3 levels: firstly by decreasing complement binding at the NMJ, secondly by protecting $A C h R$-expressing myoid cells in the thymus and thirdly by inhibition opsonisation of $\mathrm{AChR}$ immune complexes by $\mathrm{B}$-cells via complement receptors. All these processes could limit the immune-response to the AChR. Because antibody titres of MG patients are in the nano-molar range, small amounts of IgG4 would probably suffice for an effective competition with auto-antibodies, thus making it feasible to treat patients for longer periods. This would be an advantage over IVIg which is only effective in high concentrations and is usually only prescribed in severe MG because of the limited availability of donor blood.

\section{References}

Bezakova $G$, Ruegg MA. New insights into the roles of agrin. Nat Rew Mol Cell Biol 2003; 4: $295-308$.

Fatel-Moghadam A, Wick M, Besinger $U_{0}$ Geursen RG. High-dose intravenous gammagllobullin for myasthenia gravis. Lancet 1984; 1: 348-9.

Hoch W, McConville., Helms S, Newsom. Davis J, Melms A, Vincent A. Auto-antibodies to the receptor tyrosine kinase MUSK in patients with myasthenia gravis without acetylcholine receptor antibodies. Nat Med 2001; $7: 365-8$.

Jolly F: Uber myasthenta gravis pseudoparalytica. Berl. Klin. Wochenschr 1895; 32: $1-7$.

Kuncl RW, Drachman DB. Adams R, Lehar M. 3-Deazaadenosine: a therapeutic strategy for myasthenia gravis by decreasing the endocytosis of acetylcholine receptors. I Pharmacol Exp Ther 1993; 267: 582-9.

McCorville J, Farrugia ME, Beeson D, Kishore U, Metcalfe $R$, Newsom. Davis ), et al. Detection and characterization of MuSK antibodies in seronegative myasthenia gravis. Ann Neurol 2004: $55: 580$ m. 
Morgan BP. Complement membrane attack on nucleated cells: resistance, recovery and non-lethal effects. Biochem I 1989; 264: 1-14.

Mozrzymas JW. Lorenzon $P_{x}$ Riviera AP, Tedesco F, Ruzzier F. An electrophysiologieal study of the effects of myasthenia gravis sera and complement on rat isolated muscle fibres. I Neuroimmunol 1993: 45: 155-62.

Patrick I, Lindstrom I. Autoimnune response to acetylcholine receptor. Science 1973; $180 ; 871-2$.

Selcen $D_{x}$ Fukuda $T$, Shen $X M_{x}$ Engel AG. Are MuSK antibodies the primary cause of myasthenic symptoms? Neurology 2004; 62: 1945-50.

Starr MA. Myasthenia gravis. J. Nerv. Men. Dis. 1912; 39: 721-731.

Tuzun E, Scott BG, Goluszko E, Higgs $\mathrm{S}$, Christadoss $\mathbb{P}$. Genetic evidence for involvement of classical complement pathway in induction of experimental autoimmune myasthenia gravis. J Immunol 2003; 171: 3847-54.

Walker MB. Treatment of myasthenia gravis with physostigmine. Lancet 1934; 1: 1200-1. 


\section{Samenvatting en algemene discussie}

Het postsynaptische membraan van de neuromusculaire junctie (NMJ) is een zeer gespecialiseerde structuur die de betreffende spier laat samentrekken na activering door de neurotransmitter acetylcholine. Aangezien bewegingscontrole van vitaal belang is, heeft de NM] gedurende de evolutie blootgestaan aan diverse gerichte aanvallen. Toxinen tegen neuromusculaire eiwitten zijn zowell als wapen als ter verdediging ingezet. Bekende voorbeelden zijn cobragif, curare en botulinumgif. De overeenkomst tussen de symptomen die deze toxinen teweegbrengen en congenitale of verworven spierziekten heeft ertoe bijgedragen dat de oorzaak is ontdekt van ziekten bij de mens waarbij de NMJ is betrokken. De acetylcholinesteraseremmer fysostigmine, een antidotum tegen curare, was het eerste medicijn dat werd gebruikt voor de behandeling van spierzwakte bij patiënten met myasthenia gravis (MG) (Jolly, 1895; Walker, 1934). Het behandelingseffect van acetylcholinesteraseremmers is echter slechts tijdelijk en onvolledig. Thymectomie werd als behandeling wan MG toegepast nadat bij obductie van overleden MG-patiënten afwijkingen aan de thymus, zoals thymomas en folliculaire hyperplasie, waren vastgesteld (Starr, 1912). Bij sommige MG-patiënten bleek thymectomie een gunstig effect te hebben. In 1973 ontdekten Patrick en Lindstrom dat MG bij 85\% van de patiënten wordt veroorzaakt door antistoffen tegen acetylcholinereceptoren (AChR) die de NMJ aanvallen. Dit heeft geleid tot de ontwikkeling van behandelingen met immunosuppressie en plasmaferese (Patrick and Lindstrom, 1973). Beide behandelingen zijn bij de meeste MG-patiënten effectief, ook bij patiënten zonder antistoffen tegen AChR. In sommige gevallen kunnen echter ernstige bijwerkingen optreden. Ook behandeling met intraveneuze immunoglobuline (IVIg) is met succes toegepast bij MG-patiënten (Fateh-Moghadam et al.; 1984). Maar ook in combinatie is geen van deze behandelingen werkzaam bij alle MG-patiënten. Vooral MG-patiënten zonder antistoffen tegen AChR blijven moeilijk te behandelen, omdat bij hen de pathogenese niet geheel duidelijk is. De recente ontdekking van antistoffen tegen MuSK, die bij sommige, maar niet alle patiënten zonder antistoffen tegen AChR (Hoch et al., 2001) worden aangetroffen, heeft aangetoond dat MG een heterogene aandoening is met diverse oorzaken en een wisselende respons op behandeling. Een efficiëntere en meer specifieke behandeling is daaron noodzakelijk en de MG subtypen dienen verder te worden gespecificeerd. Om MG verder te onderzoeken zijn niet alleen de veranderingen van het immuunsysteem maar is ook de spierreactie van groot belang. De moleculaire samenstelling van de NMJ bepaalt de gevoeligheid voor de aanval van autoantistoffen. De interactie van $\mathrm{AChR}$ met rapsyne is bepalend voor de integriteit van het postsynaptische membraan. Gentherapie, waarmee de synaptische rapsyne-expressie wordt verhoogd, leidt tot een grotere resistentie en beter herstel van de behandelde spieren (hoofdstuk 2 en 3) in proefdieren met experimentele auto-immune myasthenia gravis (EAMG). Aangezien bij actieve, chronische 
EAMG ook een verergering wan de ziekte optrad door extrasynaptische rapsyne-expressie, is mader onderzoek noodzakelijk om eindplaatspecifieke rapsyne-expressie te garanderen en om veilige en efficiente gentransferprotocollen voor spierbehandeling vast te stellen.

Een alternatieve therapeutische benadering is behandeling met neuroprotectieve humane recombinant antistoffen (hoofdstuk 5). Een dergelijke behandeling is met de huidige biotechnologische mogelijkheden in principe op korte termijn te realiseren. Hiervoor is een AChR-specifiek humaan IgG4 geproduceerd. Dit IgG4 kan met pathogene IgGI anti$A C h R$-autoantistoffen competeren en beschadigt het postsynaptische membraan niet, waardoor het myasthenie in een niet-humaan primatenmodel kan voorkomen.

Beide bovengenoemde experimentele behandelingen kunnen alleen worden toegepast bij MG-patiënten met antistoffen tegen AChR. Patiënten met antistoffen tegen MuSK hebben een normaal $A C h R$-gehalte (Selcen et al., 2004) en een hoog IgG4-autoantistoffengehalte (Hoch et al., 2001). De antistoffen tegen MuSK hebben niet op alle spieren hetzelfde schadelijke effect. Het bepalen van de onderliggende verschillen tussen aangetaste en resistente eindplaten zou daarom kwnnen helpen om targets woor behandeling te vinden. De beschikbare diagnostische methode om antistoffen tegen MuSK te analyseren is belangrijk om de werkzaamheid van conventionele behandeling te kunnen evalueren (hoofdstuk 6). Bovendien is het van belang het klinische effect van deze antistoffen te kunnen nabootsen in een diermodel.

In hoofdstuk 1 wordt het gebruik van experimentele modellen voor MG besproken. EAMG kan bij een groot aantal diersoorten worden opgewekt door actieve AChR-immunisatie, door passieve transfer van antistoffen tegen $A C h R$, door autologe beenmergtransplantatie en cyclosporine of kan spontaan optreden. Afhankelijk van het gebruikte model treden verschillende immunologische mechanismen in werking. Bij een actief immunisam tiemodel spelen de $\mathrm{T}$-cellen een cruciale rol bij de initiatie van de productie van pathogene antistoffen antistoffen tegen $A C h R$, die complement fixeren en kunnen crosslinken. Het inspuiten van antistoffen tegen $A C h R$ is al voldoende om EAMG op te wekken. Hierbij wordt dan de rol van een specifieke, door de cel gemedieerde immunnreactie in de effectorfase van de ziekte omzeild. Oudere ratten worden door passieve overdracht en actieve immunisatie resistent tegen EAMG. Deze resistentie correleert met een verhoogd gehalte rapsyne en s-laminine (laminine $\mathrm{a} / \beta 2 \gamma 1$ ) in verhouding tot de $\mathrm{ACh}$ ter hoogte van de neuromusculaire junctie. Bij de overdracht van EAMG via beenmergtransplantatie en cy. closporine rakakt het immuunsysteem ontregelt door het ontbreken van immunisatie en kan myasthenia worden opgewekt.

In hoofdstuk 2 wordt de experimentele onderzoeksbenadering beschreven voor de rol die het postsynaptische eiwit rapsyne speelt bij de stabilisering van de AChR. Rapsyne zorgt voor clustering en verankering van $A C h R$ in het postsynaptische membraan van de NMJ. Daarom veronderstellen wij dat endogene rapsyne-expressie mogelijk een belangrijke bepalende factor is voor AChR-verlies en storing van neuromusculaire signaaloverdracht bij humane $M G$ en dat verhoging van rapsyne-expressie als behandeling kan worden toegepast. Om eerst de mogelijke therapeutische toepassingen van rapsyne te onderzoeken, heb- 
ben we acute EAMG in jonge ratten opgewekt door middel van passieve overdracht van een AChR-antistof, $m$ Ab 35, en hebben we met in wwo elektroporatie unilaterale rapsyne-overexpressie in éen spier bewerkstelligd. Vervolgens is met behulp van kwantitatieve radioimmunoassay en immunoflorescentie gekeken naar de samengestelde spieractiepotentiaal bij rapsyne- en AChR-expressie en naar de morfologie van de NMJ's, waarbij spieren met en zonder elektroporatie in EAMG-ratten en controle ratten met elkaar werden vergeleken. In de getransfecteerde spiervezels van controle ratten hebben we extrasynaptische rapsyne-aggregaten aangetroffen en licht verhoogde rapsyne- en $A C h R$-gehalten ter hoogte van de NMJ. Bü EAMG-ratten vertoonden de spieren met rapsyne-overexpressie, ondanks afzettingen van geactiveerd complement, geen afname van het spieractiepotiential, geen verlies van AChR en had de meerderheid normale postsynaptische plooien, terwijl bij de eindplaten van onbehandelde spieren typisch AChR-verlies en morfologische beschadiging optrad. Deze gegevens doen vermoeden dat de individuele verschillen in aangeboren rapsyne-expressie de bepalende factor kunnen zijn voor de ernst van de ziekte, meer dan de concentratie circulerende antistoffen tegen $\mathrm{AChR}$.

In hoofdstuk 3 wordt de mogelijkheid onderzocht om actieve, chronische EAMG te behandelen met rapsyne-overexpressie. Ondanks de aanwezigheid van hoge titers van antistoffen tegen AChR leidde rapsyne-overexpressie bij actieve, chronische EAMG tot een toename van de totale AChR-gehalten in het membraan vergeleken met de contralaterale, onbehandelde spieren. Deze toename was te zien bij eindplaten en in aggregaten van het extrasynaptische membraan. Een grote hoeveelheid antistoffen tegen AChR was aan de AChR in de celmembraan gebonden. De eindplaten in getransfecteerde EAMG-spiervezels met een hoog rapsyne gehalte herstelden van het AChR-verlies en waren te vergelijken met de eindplaten van gezonde dieren. Het gemiddelde $A C h R-g e h a l t e$ ter hoogte van de neuromusculaire juncties was echter niet veranderd vergeleken met contralaterale, onbehandelde spieren. Op ultrastructureel niveau bleek het postsynaptische membraan bij een groot aantal eindplaten in met rapsyne behandelde spieren grotere schade te hebben opgelopen. Uit deze resultaten blijkt dat extrasynaptische rapsyne het herstel van de synaps bij EAMG en mogelijk MG kan belemmeren, terwijl eindplaatspeciheke verhoging van rapsyne expressie als behandeling $\mathrm{kan}$ dienen.

In hoofdstuk 4 wordt ingegaan op de rol van studies met niet-humane primaten om therapieën voor $\mathrm{MG}$ te ontwikkelen. De filogenetische verwantschap tussen hogere primaatsoorten en de mens komt tot uitdrukking in een sterke mate van owereenkomst tussen de immunologie va beide soorten. Niet-humane primaten vormen daarom een belangrijk experimenteel model voor immunziekten bij de mens. Ziektemodellen met niet-humane primaten zijn van belang voor de preklinische ontwikkeling en evaluatie van de werkzaamheid van nieuwe therapieën, vooral behandelingen die op biologische moleculen zijn gebaseerd en vanwege de specificiteit niet bij knaagdieren kunnen worden getest. Binnen het $\mathrm{MG}$-onderzoek is actieve immunisatie met Torpedo $\mathrm{ACh} R$ en passieve overdracht wan humane antistoffen toegepast. Actieve inmmuisatie leidt tot ernstige ziekte en in sommige dieren tot respiratoir falen en overlijden. Passieve immunisatie veroorzaakt daarentegen 
lichtere en reversibele $\mathrm{MG}$-symptomen en is daarom geschilkter om het effect van antistoffern op de NMJ te onderzoeken.

Hoofdstuk 5 is een beschrijving van de experimentele therapie waarbij de bescherming van AChR tegen monokionale humane autoantistoffen in resusapen wordt getest. Het humane antigenbindende fragment Fab 637, specifiek voor de menselijke acetylcholinereceptor ( $A C h R$ ), beschermt tegen $A C h R$-verlies door de binding tussen pathogene myasthenia gravis-autoantistoffen in vitro te blokkeren. Fab 637 werd gebruikt om een volledig humaan IgG1 637 en IgG4 637 te produceren en te analyseren. Deze antistoffen binden met hoge affiniteit aan humane AChR. Uit immunohistochemisch onderzoek blijkt dat de antistoffen een kruisreactie aangaan met $A C h R$ in de spieren van resusapen, maar niet bij lagere primaten. IgG1 637 bindt en activeert complement, terwijl IgG4 637 dat niet doet. Beide recombinant antistoffen waren bivalent en wekten AChR-degradatie van TE671-cellen op door antigene modulatie. De pathogeniciteit van de antistoffen werd in een model met resusapen getest waarin EAMG passief werd overgebracht. Door IgG1 637 ontstond spierzwakte en een verlaagde respons van de samengestelde spieractiepotential na herhaalde zenuwstimulatie. Bij inspuiting heeft IgG4 637 geen pathogeen effect, zelfs niet bij de hoge concentraties die in het onderzoek werden gebruikt. Crosslinking van de AChR is dus niet voldoende, maar complementbinding is essentieel om de ziekte te kunnen opwekken.

'Toch werd opwekking van de ziekte volledig voorkomen bij dieren die $\operatorname{IgG} 4637 \mathrm{kre}$ gen toegediend voorafgaand aan de challenge met IgG1 637. Deze resultaten tonen aan dat IgGl antistoffen tegen $A C h R$ voldoende zijn om spierzwakte op te wekken en dat IgG4 de pathogene effecten van IgGl-autoantistoffen bij niet-humane primaten kan voorkomen. Deze studies geven bovendien een nieuwe benadering aan om behandelingen van $\mathrm{MG}$ patiënten te ontwikkelen.

In hoofdstuk 6 wordt het effect van antistoffen tegen MUSK op de klinische manifestatie bij seronegatieve patiènten bestudeerd. Hierbij worden de klinische kenmerken van anti-MuSK-positieve en anti-MuSK-negatieve MG-patiënten vergeleken. Het doel hiervan was vast te stellen of myasthenia gravis (MG) met antistoffen tegen MuSK een afzonderlijke subgroep van seronegatieve MG vormt. Antistoffen tegen spierspecifieke tyrosine kinase (MuSK) werden bij $55 \mathrm{MG}$-patiënten onderzocht die geen antistoffen hadden tegen acetyl cholinereceptoren. MG met antistoffen tegen MuSK kwam opvallend vaak voor bij vrouwen (15 vrouwen, twee mannen). De leeftijd bij het ontstaan van de ziekte liep uiteen van 20 tot 52 jaar, waarbij $70,6 \%$ van de patiënten jonger was dan 40 jaar. Bij de meeste patiënten $(82,4 \%)$ waren vooral de gezichtsspieren en bulbaire spieren aangedaan. Een derde van hen reageerde niet goed op anticholinesterase-middelen. Bij acht patiënten $(44,4 \%)$ bleek steroilde immunosuppressie effectief. Negen patiënten ondergingen thymectomie, zes van hen hadden geen afwijkende thymus, drie vertoonden hyperplasie van de thymus. Aan het einde van de observatieperiode waren zes patiënten $(35,3 \%)$ in remissie, vertoonden vijf patiënten $(29,4 \%)$ een verbetering, vier $(23,6 \%)$ geen verandering en waren twee patiënten $(11,7 \%)$ overleden. Hieruit blijkt dat MG-patiënten met antistoffen tegen MuSK bepaalde 
klinische kenmerken hebben die verschillen van die van andere seronegatieve $M G$-patiënten. Dit benadrukt de woorspellende waarde wan tests op antistoffen tegen MuSK bij sero. negatieve MG-patiënten.

Het ziektemechanisme van MG met antistoffen tegen MuSK wordt nog niet geheel begrepen. Een van de hoofdvragen is of beschadiging door complement ook een rol speelt bij deze vorm van MG. De hoge titers van IgG4 autoantistoffen tegen MuSK (McConville et al., 2004) en de morfologisch intacte eindplaten in biopten (Selcen et al., 2004) doen vermoeden dat het effectormechanisme totaal verschill van dat bij MG met antistoffen tegem AChR. Het zou zeer waardevol zijn on de monoklonale autoantistoffen tegen MuSK te isoleren en om de klinische kenmerken van patiënten in een diermodel te reproduceren.

Het effect van AChR-antistoffen is met behulp van het experimentele diermodel voor $\mathrm{MG}$ in detail onderzocht. In combinatie met de hiervoor genoende resultaten levert dit een steeds sterker bewijs dat de veranderingen van het postsynaptische membraan na anti-AChR-binding het gevolg zijn van een dynamische interactie tussen het immumsysteem en de spier. De spier zorgt actief voor verhoging van de omzetting van AChR en verwijdering van $\mathrm{AChR}$ uit het celmembraan dat door antilichamen is gebonden (antigene modulatie).

Dit effect van antistoffen tegen AChR is op zich onwoldoende om de NMJ te beschadigen (hoofdstuk 5) en het is nog niet duidelijk of het een essentiële stap is in de pathogenese. Wanneer humaan IgG1 of IgG3 bindt aan AChR in de NMJ vindt verdere crosslinking plaats van de antistoffen door complementmolecuul Clq. Deze stap leidt niet direct tot de ziekte maar activeert de klassieke cascade van het complementsysteem, waardoor membrane attack complexes (MAC) in het postsynaptische membraan gevormd worden (Tuzun et al., 2003). Het MAC vormt een porie in het membraan waardoor verlies van cytoplasmaeiwitten en depolarisatie van het membraan optreedt. Het MAC is bedoeld om pathogenen te doden, maar door binding aan autoantistoffen kan het ook depolarisatie van het postsynaptische membraan veroorzaken (Mozrzymas et al., 1993).

Toch kunnen celkern bezittende cellen autoloog complenent uit een membraan verwijderen door vesiculatie van MAC-bevattende membranen. Omdat er bij AChR-degra datie sprake is van endocytose (Kuncl et al., 1993), is het mogelijk dat dit mechanisme verantwoordelijk is voor het verlies van postsynaptische plooiing en AChR-verlies (Morgan, 1989). De mate van $A C h R$-verlies en beschadiging van het postsynaptische membraan door MAC hangt sterk af van de verankering van de membraaneiwitten an het cytoskelet en de basale lamina. AChR wordt in het membraan geclusterd door rapsyne, dat weer via $\beta$-dystroglycan, utrofine en $\mathrm{F}$-actine gekoppeld is aan het cytoskelet (hoodtstuk 1 , figuur 1 , pagina 21). Bovendien is $\beta$-dystroglycan via a-dystroglycan en s-laminine aan het basale membraan gekoppeld (Bezakova and Ruegg, 2003). Door verhoogde rapsyne-expressie wordt de NMI gestabiliseerd en de resistentie tegen complement vergroot (general discussion, figuur 1 , pagina 111). Rapsyne-expressie bepaalt de mate van beschadiging aan de NMJ nadat de antistoffen aan AChR binden (hoofdstuk 2). Deze hypothese kan een verklaring zijn voor het feit dat sommige spieren bij $M G$-patiënten sterker worden aangetast 
dan andere. Zo kunnen de verschillen in rapsyne-expressie ook de ernst van de ziekte bij MG patienten beinvloeden en verklaren waarom er geen goede correlatie is tussen de titers. van antistoffen tegen $A C h R$ en de klinische symptomen. Het zou daarom interessant zijn te onderzoken of rapsyne-expressie door promotor polymorfismen wordt beinvloed en hoe de promotor van rapsyne in verschillende spieren en individuen wordt gereguleerd door transcriptiefactoren als Kaiso.

Verhoogde rapsyne-expressie kan daarnaast ook bijdragen aan het herstel wan het AChR-gehalte van een beschadigde NMJ bij actieve EAMG (hoofdstuk 3). Bij aanwezigheid van attoantistoffen wordt alleen door synaptische rapsyne-expressie de AChR-gehalten in het postsynaptische membraan werhoogd. Het zou daarom interessant zijn onderzoek te verrichten naar het therapeutische effect van rapsyne-expressie onder controle van de eindplaat-specifieke promotor van de AChR-c-subunit.

Aangezien antistoffen tegen AChR de NMJ niet direct beschadigen maar een pathogeen effect opwekken via de MAC, kan preventie van complementbinding aan de eindplaat mogelijk een behandeling zijn bij MG. Humaan IgG4 bindt geen complement en AChRspecifieke IgG4 verhindert de binding van IgG1- (en waarschijnlijk ook van IgG3-) antistoffen (hoofdstuk 5). Dit effect kan op drie niveaus een therapeutische werking hebben: allereerst door de complementbinding ter hoogte van de NMJ te verminderen, ten tweede door de AChR-expressie van myoide cellen in de thymus te beschermen en ten derde door de opsonisatie van $A C h R$-immunucomplexen door B-cellen via complementreceptoren te remmen. Al deze processen kunnen de immunureactie op $\mathrm{AChR}$ beperken. Omdat de antistoftiters bij MG-patiènten in het nanomolaire bereik liggen, zullen kleine hoeveelheden IgG4 waarschijnlijk voldoende zijn voor een effectieve competitie met autoantistoften. Hierdoor is het mogelijk om patiënten gedurende langere tijd te behandelen. Dit zou een voordeel kunnen zijn ten opzichte van IVIg, dat alleen in hoge concentratie effectief is en meestal slechts bij ernstige MG wordt voorgeschreven in verband met het beperkte aanbod van donorbloed.

\section{Referenties}

Berakova G, Ruegg MA. New insights into the roles of agrin. Nat Rev Mol Cell Biol 2003; 4: 295-308.

Fateh-Moghadam A, Wick M, Besinger U, Geursen RG. High - dose intravenous gammaglobulin for myasthenia grawis. Lancet $1984 ; 1: 848-9$.

Hocln W, McConville d, Helns S, Newsom-Dawis I, Melms A, Vincent A. Auto-antibodies to the receptor tyrosine kintse MuSK in patients with myasthenia gravis without acetylcholine receptor antibodies. Nat Med 2001; $7: 365-8$

Jolly l: Uber myasthenia gravis pseudoparalytica. Berl. Klin. Wochenschr 1895; 32: 1-7.

Kuncl RW, Drachman DB, Adams R, Lehar M. 3m Deazadenosine; a therapentic strategy for myasthenia gravis by decreasing the endocytosis of acetylcholine receptors. J Pharmacol Exp Ther 1993; 267: 582-9.

Morgan BP. Complement membrane attack on nucleated cells: resistance, recovery and non-lethal effects. Biochem ) 1989, 264: 1-14. 
Morrymas JW, Lorenzon P, Riviera AP, Tedesco F Ruzzier A. An electrophysiological study of the eftects of myas thenia gravis sera and complement on rat isolated muscle fobres. I Neuroimminol $1993,45: 155-62$.

Patrick J, Lindstrom 1. Autoimmune response to acetylcholine receptor. Science 1973, 180: 871-2.

Selcen D, Fukuda T, Shen XM, Engel AG. Are MuSK antibodies the primary cause of mysthenic symptoms? Neurology 2004: 62: 1945-50.

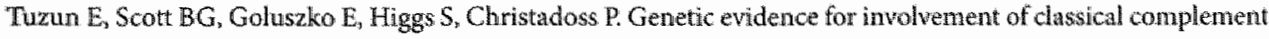
pathway in induction of experimental autoimmume myasthenia gravis.. I Immunol 2003: 171: 3847-54.

Walker MB. Treatment of myasthenia gravis with physostigmine. Lancet 1934 ; 1 : 1200-1. 


\section{Publications}

\section{Research articles}

Losen M', Martínez-Martínez P', 't Hart BA, Brok HPM, Spaans F, Duimel H, Frederik P, Verschuuren JIGM, van Dijk G, Toyka KV, Fostieri E, Tzartos SJ, Berrih-Aknin S, Bosmans E, Houtkamp M, Wiegman L, Vink T, Grats Y, Schuurman I, Parren PWHI and De Baets MH. Passive transfer of a human IgG4 anti-AChR antibody prevents experimental autoimmune myasthenia gravis in rhesus monkeys. Submitted.

Losen $\mathrm{M}^{*}$, Martínez-Martínez $\mathrm{P}^{*}$, Duimel H, Frederik P, Spaans F, Molenaar P, Vincent A and De Baets $\mathrm{MH}$. Overexpression of rapsyn in rat muscle increases acetylcholine receptor levels in chronic experimental autoimmune myasthenia gravis. Submitted.

Losen M, Stassen MH, Martinez-Martínez P, Machiels BM, Duimel H, Frederik P, Veldman $\mathrm{H}$, Wokke JH, Spaans F, Vincent A, De Baets MH. Increased expression of rapsyn in muscles prevents acetylcholine receptor loss in experimental autoimmune myasthenia gravis. Brain, 2005; 128:2327-37

Lavrnic D, Losen M, Vujic A, De Baets M, Hajdukovic LJ, Stojanovic V, Tríkic R, Djukic P, Apostolski S. The features of myasthenia gravis with autoantibodies to MuSK. I Neurol Neurosurg Psychiatry. 2005; 76(8):1099-102.

Roorda BD, Hesselink MK, Schaart G, Moonen-Kornips $\mathbb{E}$, Martinez-Martinez $\mathrm{P}$, Losen M, De Baets MH, Mensink RP, Schrawwen P. DGATl overexpression in muscle by in vivo DNA electroporation increases intramyocellular lipid content. J Lipid Res. 2005; 46(2):230-6.

Losen M, Frolich B, Pohl M, Buchs J. Effect of oxygen limitation and medium composition on Escherichia coli fermentation in shake-flask cultures. Biotechnol Prog. 2004; 20(4):1062-8.

Stockmann C, Losen M, Dahlems U, Knocke C, Gellissen G, Buchs J. Effect of oxygen supply on passaging, stabilising and screening of recombinant Hansenula polymorpha production strains in test tube cultures. FEMS Yeast Res. 2003; 4(2):195-205.

Maier U, Losen M, Buchs J. Advances in understanding and modelling the gas-liquid mass transfer in shake flasks. J Biochem Eng. 2003; 17(3):155-167. 


\section{Reviews}

De Baets M, Stassen M, Losen M, Zhang X, Machiels B. Immunoregulation in experimental autoimmune myasthenia gravis-about $T$ cells, antibodies, and endplates. Ann $N Y$ Acad Sci. 2003; 998:308-17.

\section{Book chapters}

't Hart BA, Losen M, Brok HPM, de Baets MH., Chronic Diseases. In: The llaboratory primate. Wolfe-Coote SP, ed. London: Elsevier, 2005; 417-36.

\section{Abstracts}

Losen M, Martínez-Martínez P, Machiels BM De Baets M. Recombinant antibodies for MG therapy. Joint annual meeting for immunology of the Dutch and German societies of immunology (NVVI, DGfI), October 2004, Maastricht, The Netherlands.

Losen M, Martínez-Martinez P, Machiels BM De Baets M. Recombinant antibodies for MG therapy. Myology, May 2005, Nantes, France.

Martinez-Martinez P, Losen M, van de Waarenburg M, Machiels BM De Baets M. Rapsyn gene therapy of experimentall autoimmune myasthenia gravis. Myology, May 2005, Nantes, France.

Losen M, Martinez-Martínez P, Stassen MHW, Machiels BM, Tzartos SI, Berrih-Aknin S, Roos A, Daha MR, Bosmans E, "t Hart B, Brok H, Schuurman J, Parren PWHII and De Baets MH. Recombinant antibodies for MG therapy. 7th International Congress of Neuroimmunology, September 2004, Venice, Italy. I Neuroimmunology. 2004; 154:153

Stöckmann C, Losen M, Dahlems U, Gellissen G, Büchs I. Festlegung reproduzierbarer Screening-Bedingungen - Fallbeispiel Hansenula polymorpha. DECHEMA/GVCJahrestagung 2002, Wiesbaden, Germany.

Losen $M$, Stöckmann $C$, Anderlei T, Hermann $R$, Maier $U_{n}$ Hellwig $S$, Lingen $B$, Pohl $M$, $B$ iichs J Culture conditions in the screening of microorganisms - Importance of oxygen supply, Bioforum 2001, Liege, Belgium.

M. Losen, C. Stöckmann, T. Anderlei, R. Hermann, U. Maier, S. Hellwig, B. Lingen, M. Pohl, J. Büchs, Sauerstoff im Screening von Mikroorganismen - Problematik der Sauerstoffversorgung, Dechema 2001, Leipzig, Germany. 


\section{Presentations}

Losen M, Martinez-Martínez P, De Baets MH. The role of rapsyn in myasthenia gravis. Endo-Neuro-Psycho Meeting, May 2005, Doorwerth, The Netherlands.

Losen M, Martinez-Martinez P. De Baets MH. Molecular approaches to therapies of neuroimmunological diseases, 4 th Institute's Day of the Research Institute of Brain and Behaviour, January 21, Maastricht, The Netherlands.

Martinez-Martínez P, Losen M, Stassen MHW, Machiels BM, Veldman H. Wokke JHI, Spaans $\mathrm{F}_{x}$ De Baets. Rapsyn gene therapy of experimental autoimmune myasthenia gravis. Joint annual meeting for immunology of the Dutch and German societies of immunology (NVVI, DGfI), October 2004, Maastricht, The Netherlands.

Martinez-Martinez P, Losen M, Stassen MHW, Machiels BM, Veldman H. Wokke $J H]_{\text {s }}$ Spaans $\mathbb{E}$, De Baets $M$. Rapsyn gene therapy of experimental autoimmune myasthenia gravis. 7th International Congress of Neuroimmunology, September 2004, Venice, Italy. J Neuroimmunology. 2004; 154:142.

Martínez-Martínez P, Losen M, Stassen MHW, Machiels BM, Veldman H. Wokke JH), Spaans F, De Baets Rapsyn gene therapy of experimental autoimmune myasthenia gravis. AFM/INSERM Myasthenia gravis meeting, April 2004, Athens, Greece.

Lavrnic D, Losen M, De Baets M, Vujic A, Hajdukovic LJ, Stojanovic V, Trikic R, Djukic P, Apostolski S. The features of myasthenia gravis with anti-MuSK antibodies. AFM/ INSERM Myasthenia grawis meeting, April 2004, Athens, Greece. 


\section{Curriculum Vitae}

Mario René Losen was born in Haselünne, Germany, on August 21, 1974. He finished his secondary education at the Kreisgymnasium Heinsberg in 1994. He studied Biology at the Rheinisch-Westfälische Technische Hochschule Aachen (RWTH) and the Imperial College of Science, Technology and Medicine London and graduated in 2001. In 2001 he worked as scientific assistant in the Department of Biochemical Engineering of Prof. Jochen Büchs at the RWTH Aachen. During this year he analysed the metabolism of industrial strains of microorganisms in collaboration with the Institute of Enzyme Technology at the Forschungszentrum Jülich and the Rheinbiotech $\mathrm{GmbH}$ in Düsseldorf. In 2002 he started his PhD training on a project funded by the European Union with the objective to develop "Innovative therapeutic strategies for the prototype autoimmune disease, myasthenia gravis" in the Department of Neurology of the University of Maastricht. Two novel experimental therapeutic approaches were developed for the treatment of myasthenia gravis, which are described in this thesis. Moreover he wrote or contributed major parts to several awarded grant applications funded by the European Union, the School of Life Sciences of the Transnational University Limburg, the Princess Beatrix Fonds, the Association Française contre les Myopathies, Genmab, Debiovision and Aspreva. In 2006 he received a Kootstra fellowship for talented graduates of the Faculty of Medicine at the University of Maastricht. Currently he works on the development of immunotherapies for neurological diseases in the Division of Cellular Neuroscience at the University of Maastricht. 


\section{Acknowledgments}

Many of the experiments and results described in this thesis are the result of a collaborative and interdisciplinary effort and I am very grateful to all the friendly, enthusiastic, helpful and creative people I have had the privilege to work with.

In particular, I would to thank my supervisor Dr. Marc De Baets who with his clinical and research experience, his knowledge of autoimmune diseases and his enthusiasm to develop new experimental approaches has introduced me to many facets of biomedical research. With his ideas and constructive planning he has very efficiently guided my research using experimental models of myasthenia gravis. Moreover, he gave me the opportunity travel to a large number of international conferences and to learn the ins and outs of writing grant applications.

I am grateful to Prof. Harry Steinbusch for giving me the opportunity to work in the Institute of Brain and Behaviour, his effort to integrate my work in the general research lines of the Division of Cellular Neuroscience and his guidance especially in the final year of my $\mathrm{PhD}$ project.

I would like to thank Dr. Piluca Martinez for her advice on molecular biology techniques, her enthusiasm and her constant help.

I have very much enjoyed the fruitful discussions about possible gene- and immunotherapies for myasthenia gravis and performing experiments with the co-authors of the articles in this thesis. Their expertise has been indispensable for the completion of this work. Additionally, I would like to thank Dr. Oliver Bauer, Dr. Wim Engels, Prof. Christian Fuhrer, Dr. Laurent Schaeffer and Dr. Chistoph Schmitz for their expert advice.

Moreover, I appreciate the help I received from the technicians Hans Duimel, Wiel Honig, Dr Barbie Machiels, Marjanne Markerink, Hellen Steinbusch and Marjo van de Waarenburg, the office staff Nicole Senden, Akke Spauwen, Lilian Swaen and Mirèse Willems.

I have enjoyed the collegiality of the Brain and Behaviour PhD students Matteo Bruschettini, Dr. Ronald Deumens, Roselie Diederen, Erik Hoff, Dr. Daniel van den Hove, Dr. Gunther Kenis, Guido Koopmans, Pawel Kreczmanski, Evi Lemmens, Ayemu Myint, Marko Phernambucq, Dr. Bart Rutten, Dr. Monica Sica and Yasin Temel.

I would like to acknowledge the work and interest of the students that worked on diverse projects for developing recombinant antibodies: Paul Essers, Joris van Houte, Talia Latuhihin, Angela Spiertz, Matthijs van de Vrie and Rick Wetzels.

Ten slot wil ik alle familie en vrienden bedanken, die gedurende de afgelopen jaren met mij hebben meegeleefd, speciaal mijn Oma Pietje. 
Ganz besonders möchte ich mich bei meiner Familie für alle Unterstützung, Rat und Sorge bedanken, die sie mir in den letzten Jahren gegeben hat. Lieber Papa, Mama, Jan, Oma Emmi, Piluca und Sören, ihr wart immer für mich da und habt mir die Daumen gedrückt, und es hat geholfen! 
